Supporting Information

\title{
Facile Mechanochemical Anion Substitution in Cyclopalladated Azobenzenes
}

\author{
Alen Bjelopetrović, Marko Robić, Ivan Halasz, Darko Babić, Marina Juribašić Kulcsár* \\ and Manda Ćurić* \\ Ruđer Bošković Institute, Bijenička 54, HR-10000 Zagreb, Croatia
}

\section{Table of Contents}

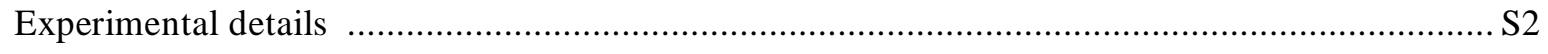

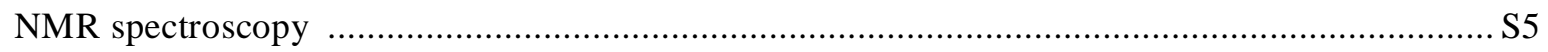

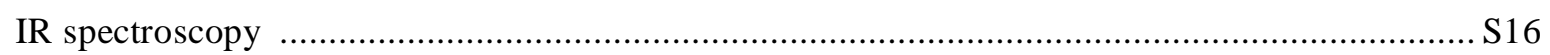

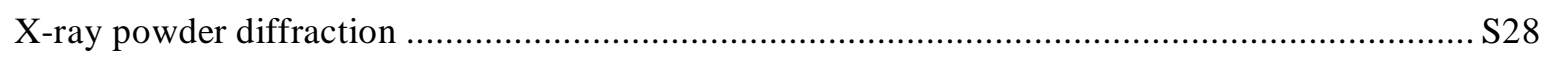

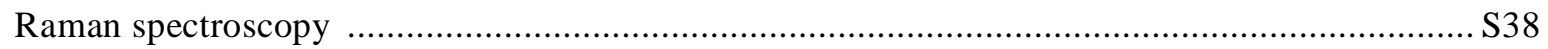

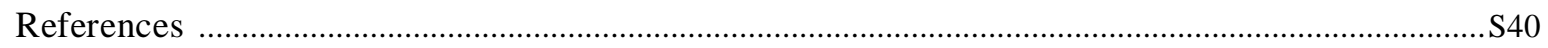




\section{EXPERIMENTAL DETAILS}

General Methods. All chemicals were used as supplied and were not additionally purified or dried. The ${ }^{1} \mathrm{H}$ NMR spectra were recorded at $25{ }^{\circ} \mathrm{C}$ in $\mathrm{CDCl}_{3}$ and/or in DMSO- $d_{6}$ with Brucker's spectrometers AV-600, AV-400 and AV-300. FTIR spectra were recorded with Perkin-Elmer's spectrometer Spectrum Two. PXRD measurements were performed on a PANalytical Aeris X-ray diffractometer with Ni-filtered $\mathrm{Cu} K_{\alpha}$ radiation. Elemental analyses were done on a Perkin-Elmer Series II 2400 CHNS/O analyzer.

\section{Ball-milling reactions}

Ball-milling experiments were performed at room temperature of $22 \pm 2{ }^{\circ} \mathrm{C}$ in the $14 \mathrm{~mL}$ poly(methylmetacrylate) (PMMA) jars using one $12 \mathrm{~mm}(4.3 \mathrm{~g}) \mathrm{ZrO}_{2}$ milling ball. An IST500 mixer mill with a built-in fan operating at $30 \mathrm{~Hz}$ frequency was used for the synthesis. All reactions were carried out under similar conditions starting with the total weight of reaction mixture of about $220 \mathrm{mg}$.

\section{Initial compounds}

Palladium precursors $\left[(\mathrm{L} 1) \mathrm{Pd}(\mathrm{MeCN})_{2}\right]\left[\mathrm{BF}_{4}\right]$ (1), [(L2)Pd(MeCN $\left.)_{2}\right]\left[\mathrm{BF}_{4}\right]$ (2), [(L2)Pd( $\mu$ $\mathrm{OAc})]_{2}(4),\left[\mathrm{Pd}_{2}\left(\mathrm{H}_{2} \mathrm{O}\right)(\mu-\mathrm{Cl})(\mu-\mathrm{L} 1)(\mathrm{Cl})\right]_{2}(\mathbf{9}),\left[\mathrm{Pd}_{2}\left(\mathrm{H}_{2} \mathrm{O}\right)(\mu-\mathrm{Cl})(\mu-\mathrm{L} 2)(\mathrm{Cl})\right]_{2}(10)$ and $[\mathrm{Pd}(\mu-$ $\left.\mathrm{OAc})_{2}(\mu-\mathrm{L} 2) \mathrm{Pd}\right]_{2} \quad(\mathbf{1 2}), \quad$ where $\mathrm{L} 1=$ azobenzene and $\mathrm{L} 2=4$-chloro $-4{ }^{\prime}-(N, N-$ dimethylamino)azobenzene, were prepared by using the ball-milling procedures recently developed by our group. ${ }^{1}$ Only the precursor $[(\mathrm{L} 1) \mathrm{Pd}(\mu-\mathrm{OAc})]_{2}(3)$ was prepared by the aging of solid $\left[\mathrm{Pd}(\mathrm{OAc})_{2}\right]_{3}$ and L1 in DMF vapour. ${ }^{2}$ Precursors 1 and 9 were prepared for the first time via mechanochemical $\mathrm{C}-\mathrm{H}$ bond activation.

[(L1)Pd(MeCN) $\left.)_{2}\right]\left[\mathrm{BF}_{4}\right]$ 1. Mixture of L1 $(55.00 \mathrm{mg}, 0.30 \mathrm{mmol}),\left[\mathrm{Pd}\left(\mathrm{CH}_{3} \mathrm{CN}\right)_{4}\right]\left[\mathrm{BF}_{4}\right]_{2}$ (134.19 mg, $0.30 \mathrm{mmol}), \mathrm{NaOAc}(24.77 \mathrm{mg}, 0.30 \mathrm{mmol})$ and $\mathrm{NaBF}_{4}(27.70 \mathrm{mg}, 0.25 \mathrm{mmol})$ were milled for 60 minutes. The crude product was washed with $\mathrm{H}_{2} \mathrm{O}$. Isolated yield $80 \%$. Found: C 41.73, H 2.97, N 12.48; Calcd. for $\mathrm{C}_{16} \mathrm{H}_{15} \mathrm{BF}_{4} \mathrm{~N}_{4} \mathrm{Pd}$ : C 42.09, H 3.31, N 12.27.

$\left[\mathbf{P d}_{2}\left(\mathbf{H}_{2} \mathbf{O}\right)(\boldsymbol{\mu}-\mathbf{C l})(\boldsymbol{\mu}-\mathbf{L 1})(\mathbf{C l})\right]_{2}$ 9. Mixture of L1 (40.00 mg , $\left.0.22 \mathrm{mmol}\right), \mathrm{PdCl}_{2}(155.83 \mathrm{mg}$, $0.88 \mathrm{mmol}$ ), $\mathrm{NaOAc}\left(36.04 \mathrm{mg}, 0.44 \mathrm{mmol}\right.$ ) and $15 \mu \mathrm{L}$ of $\mathrm{H}_{2} \mathrm{O}$ were milled for 36 hours. The crude product was washed with $\mathrm{H}_{2} \mathrm{O}$ and acetone. Isolated yield $83 \%$. Found: $\mathrm{C} 38.13, \mathrm{H}$ 2.29, N 7.09; Calcd. for $\mathrm{C}_{24} \mathrm{H}_{20} \mathrm{Cl}_{4} \mathrm{~N}_{4} \mathrm{O}_{2} \mathrm{Pd}_{2}$ : C 38.38, H 2.68, N 7.46.

\section{Mechanosynthesis of mono- and dipalladated complexes via anion substitution}

All reactions were performed with one equivalent of mono- and dipalladated L1 or L2 (acetate, chloride or tetrafluoroborate complexes) and 10 equivalents of alkali salts $\mathrm{MX}$ $(\mathrm{M}=\mathrm{Li}$ or $\mathrm{Na})$ of acetate $(\mathrm{OAc})$, acetylacetonate (acac) and chloride $(\mathrm{Cl})$ per one palladium centre using neat grinding (NG). The resulting mixtures were washed with water and dried under vacuum. Reaction times are given in Table 1 of the main manuscript.

\section{Synthesis of acetylacetonate complexes via anion substitution}

\{(L1)Pd(acac)\} 7. a) $67.00 \mathrm{mg}(0.15 \mathrm{mmol})$ of $\left[(\mathrm{L} 1) \mathrm{Pd}(\mathrm{MeCN})_{2}\right]\left[\mathrm{BF}_{4}\right]$ (1) and $155.63 \mathrm{mg}$ (1.47 mmol) of $\mathrm{Li}$ (acac). Isolated yield 85\%. Found: C 53.18, H 4.34, N 7.28; Calcd. for $\mathrm{C}_{17} \mathrm{H}_{16} \mathrm{~N}_{2} \mathrm{O}_{2} \mathrm{Pd}$ : C 52.80, $\mathrm{H} 4.17, \mathrm{~N} 7.24$. b) $55.00 \mathrm{mg}(0.08 \mathrm{mmol})$ of $[(\mathrm{L} 1) \mathrm{Pd}(\mu-\mathrm{OAc})]_{2}(3)$ 
and $168.25 \mathrm{mg}(1.58 \mathrm{mmol})$ of $\mathrm{Li}(\mathrm{acac})$. Isolated yield 85\%. Found: C 52.48, H 4.28, N 7.39. c) $52.00 \mathrm{mg}(0.08 \mathrm{mmol})$ of $[(\mathrm{L} 1) \mathrm{Pd}(\mu-\mathrm{Cl})]_{2}(5)$ and $170.68 \mathrm{mg}(1.61 \mathrm{mmol})$ of $\mathrm{Li}(\mathrm{acac})$. Isolated yield 92\%. Found: C 52.51, H 4.05, N 7.17.

\{(L2)Pd(acac)\} 8. a) $75.00 \mathrm{mg}(0.14 \mathrm{mmol})$ of $\left[(\mathrm{L} 2) \mathrm{Pd}(\mathrm{MeCN})_{2}\right]\left[\mathrm{BF}_{4}\right]$ (2) and $148.93 \mathrm{mg}$ (1.40 mmol) of $\mathrm{Li}(\mathrm{acac})$. Isolated yield 94\%. Found: $\mathrm{C} 49.51, \mathrm{H} \mathrm{4.64,} \mathrm{N} \mathrm{8.95;} \mathrm{Calcd.} \mathrm{for}$ $\mathrm{C}_{19} \mathrm{H}_{20} \mathrm{ClN}_{3} \mathrm{O}_{2} \mathrm{Pd}: \mathrm{C} 49.15, \mathrm{H} 4.34, \mathrm{~N} 9.05$. b) $63.00 \mathrm{mg}(0.07 \mathrm{mmol})$ of $[(\mathrm{L} 2) \mathrm{Pd}(\mu-\mathrm{OAc})]_{2}(4)$ and $157.50 \mathrm{mg}(1.48 \mathrm{mmol})$ of $\mathrm{Li}(\mathrm{acac})$. Isolated yield 88\%. Found: C 48.91, H 4.38, N 8.91. c) $61.00 \mathrm{mg}(0.07 \mathrm{mmol})$ of $[(\mathrm{L} 2) \mathrm{Pd}(\mu-\mathrm{Cl})]_{2}(6)$ and $161.48 \mathrm{mg}(1.52 \mathrm{mmol})$ of $\mathrm{Li}(\mathrm{acac})$. Isolated yield 89\%. Found: C 49.45, H 4.02, N 9.38. Isolated yield 89\%. Found: C 49.45, H 4.02, N 9.38 .

$\{(\mathbf{a c a c}) \mathbf{P d}(\boldsymbol{\mu}-\mathbf{L} 1) \mathbf{P d}(\mathbf{a c a c})\} \mathbf{1 3}^{- \text {a) }} 43.00 \mathrm{mg}(0.04 \mathrm{mmol})$ of $\left[\mathrm{Pd}(\mu-\mathrm{OAc})_{2}(\mu-\mathrm{L} 1) \mathrm{Pd}\right]_{2}(\mathbf{1 1})$ and $178.43 \mathrm{mg}(1.68 \mathrm{mmol})$ of $\mathrm{Li}(\mathrm{acac})$. Isolated yield 85\%. Found: $\mathrm{C} 44.35, \mathrm{H} \mathrm{3.61,} \mathrm{N} \mathrm{4.93.}$ Calcd. for $\mathrm{C}_{22} \mathrm{H}_{22} \mathrm{~N}_{2} \mathrm{O}_{4} \mathrm{Pd}_{2}$ : C 44.69, $\mathrm{H} 3.75, \mathrm{~N} 4.74$. b) $33.00 \mathrm{mg}(0.04 \mathrm{mmol})$ of $\left[\mathrm{Pd}_{2}\left(\mathrm{H}_{2} \mathrm{O}\right)(\mu-\mathrm{Cl})(\mu-\mathrm{L} 1)(\mathrm{Cl})\right]_{2}(9)$ and $186.38 \mathrm{mg}(1.76 \mathrm{mmol})$ of $\mathrm{Li}(\mathrm{acac})$. Isolated yield $86 \%$. Found: C 44.37, H 3.53, N 4.88.

$\{(\mathbf{a c a c}) \mathbf{P d}(\boldsymbol{\mu}-\mathbf{L} 2) \mathbf{P d}(\mathbf{a c a c})\}$ 14. a) $48.00 \mathrm{mg}(0.04 \mathrm{mmol})$ of $\left[\mathrm{Pd}(\mu-\mathrm{OAc})_{2}(\mu-\mathrm{L} 2) \mathrm{Pd}\right]_{2}(\mathbf{1 2})$ and 172.95 (1.63 mmol) $\mathrm{mg}$ of $\mathrm{Li}(\mathrm{acac})$. Isolated yield 92\%. Found: C 43.25, H 3.52, N 6.11. Calcd. for $\mathrm{C}_{24} \mathrm{H}_{26} \mathrm{ClN}_{3} \mathrm{O}_{4} \mathrm{Pd}_{2}$ : C 43.10, H 3.92, $\mathrm{N} 6.28$. b) $46.00 \mathrm{mg}(0.04 \mathrm{mmol})$ of $\left[\mathrm{Pd}_{2}\left(\mathrm{H}_{2} \mathrm{O}\right)(\mu-\mathrm{Cl})(\mu-\mathrm{L} 2)(\mathrm{Cl})\right]_{2}(\mathbf{1 0})$ and $174.38 \mathrm{mg}(1.64 \mathrm{mmol})$ of $\mathrm{Li}(\mathrm{acac})$. Isolated yield 86\%. Found: C 43.16, H 4.07, N 6.12.

\section{Synthesis of acetate complexes via anion substitution}

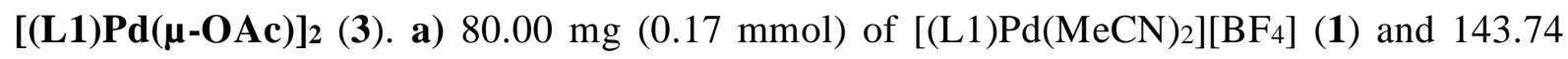
$\mathrm{mg}(1.75 \mathrm{mmol})$ of $\mathrm{NaOAc}$. Isolated yield $87 \%$. Found: $\mathrm{C} 48.25, \mathrm{H} \mathrm{3.68}, \mathrm{N} \mathrm{7.85}$. Calcd. for $\mathrm{C}_{28} \mathrm{H}_{24} \mathrm{~N}_{4} \mathrm{O}_{4} \mathrm{Pd}_{2}: \mathrm{C} 48.50, \mathrm{H} 3.49, \mathrm{~N} 8.08$. b) $62.00 \mathrm{mg}(0.09 \mathrm{mmol})$ of $[(\mathrm{L} 1) \mathrm{Pd}(\mu-\mathrm{Cl})]_{2}(5)$ and $157.41 \mathrm{mg}(1.92 \mathrm{mmol})$ of $\mathrm{NaOAc}$. Isolated yield 88\%. Found: $\mathrm{C} 48.82, \mathrm{H} \mathrm{3.56, \textrm {N }} 7.77$. c) $70.00 \mathrm{mg}(0.18 \mathrm{mmol})$ of $\{(\mathrm{L} 1) \mathrm{Pd}(\mathrm{acac})\}(7)$ and $148.47 \mathrm{mg}(1.81 \mathrm{mmol})$ of $\mathrm{NaOAc}$. Isolated yield 91\%. Found: C 48.15, H 3.58, N 7.74.

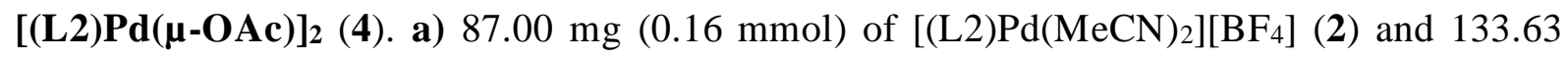
$\mathrm{mg}(1.63 \mathrm{mmol}) \mathrm{NaOAc}$. Isolated yield 93\%. Found: C 44.97, H 3.44, N 9.66. Calcd. for $\mathrm{C}_{32} \mathrm{H}_{32} \mathrm{Cl}_{2} \mathrm{~N}_{6} \mathrm{O}_{4} \mathrm{Pd}_{2}: \mathrm{C} 45.30, \mathrm{H} 3.80, \mathrm{~N} 9.91$. b) $73.00 \mathrm{mg}(0.09 \mathrm{mmol})$ of $[(\mathrm{L} 2) \mathrm{Pd}(\mu-\mathrm{Cl})]_{2}(6)$ and $149.48 \mathrm{mg}(1.82 \mathrm{mmol})$ of NaOAc. Isolated yield 87\%. Found: C 45.67, H 3.64, N 9.97. c) $80.00 \mathrm{mg}(0.17 \mathrm{mmol})$ of $\{(\mathrm{L} 2) \mathrm{Pd}(\mathrm{acac})\}(\mathbf{8})$ and $141.35 \mathrm{mg}(1.72 \mathrm{mmol})$ of NaOAc. Isolated yield 90\%. Found: C 44.98, H 3.69, N 9.56.

[Pd( $\boldsymbol{\mu}$-OAc) $2(\boldsymbol{\mu}-\mathbf{L 1}) \mathbf{P d}]_{2}(\mathbf{1 1})$. a) $41.00 \mathrm{mg}(0.05 \mathrm{mmol})$ of $\left[\mathrm{Pd}_{2}\left(\mathrm{H}_{2} \mathrm{O}\right)(\mu-\mathrm{Cl})(\mu-\mathrm{Ll})(\mathrm{Cl})\right]_{2}(\mathbf{9})$ and $179.11 \mathrm{mg}(2.18 \mathrm{mmol})$ of NaOAc. Isolated yield 88\%. Found: $\mathrm{C} 37.33, \mathrm{H} \mathrm{2.58, \textrm {N }} 5.28$. Calcd. for $\mathrm{C}_{32} \mathrm{H}_{28} \mathrm{~N}_{4} \mathrm{O}_{8} \mathrm{Pd}_{4}$ : C 37.60, $\mathrm{H}$ 2.76, N 5.48. b) $60.00 \mathrm{mg}(0.10 \mathrm{mmol})$ of $\{(\mathrm{acac}) \mathrm{Pd}(\mu-\mathrm{L} 1) \mathrm{Pd}(\mathrm{acac}\}(\mathbf{1 3})$ and $166.48(2.02 \mathrm{mmol})$ of $\mathrm{NaOAc}$. Isolated yield $90 \%$. Found: C 37.31, H 2.38, N 5.56.

$\left[\mathbf{P d}(\boldsymbol{\mu} \text {-OAc })_{2}(\boldsymbol{\mu}-\mathbf{L} 2-2 \mathrm{H}) \mathbf{P d}\right]_{2}(\mathbf{1 2})$. a) $56.00 \mathrm{mg}(0.05 \mathrm{mmol})$ of $\left[\mathrm{Pd}_{2}\left(\mathrm{H}_{2} \mathrm{O}\right)(\mu-\mathrm{Cl})(\mu-\mathrm{L} 2)(\mathrm{Cl})\right]_{2}$ (10) and $164.21 \mathrm{mg}(2.00 \mathrm{mmol})$ of $\mathrm{NaOAc}$. Isolated yield 88\%. Found: C 36.98, H 2.92, N 7.36. Calcd. for $\mathrm{C}_{36} \mathrm{H}_{36} \mathrm{Cl}_{2} \mathrm{~N}_{6} \mathrm{O}_{8} \mathrm{Pd}_{4}$ : C 36.73, H 3.08, N 7.14. b) $65.00 \mathrm{mg}(0.10 \mathrm{mmol})$ of 
$\{(\mathrm{acac}) \mathrm{Pd}(\mu-\mathrm{L} 2) \mathrm{Pd}(\mathrm{acac})\}(\mathbf{1 4})$ and $159.45(1.94 \mathrm{mmol})$ of NaOAc. Isolated yield $92 \%$. Found: C 37.02, H 3.28, N 7.37.

\section{Synthesis of chloride complexes via anion substitution}

[(L1)Pd( $\boldsymbol{\mu}-\mathbf{C l})]_{2}$ (5). a) $115.00 \mathrm{mg}(0.25 \mathrm{mmol})$ of [(L1)Pd(MeCN)$\left.)_{2}\right]\left[\mathrm{BF}_{4}\right](\mathbf{1})$ and $106.78 \mathrm{mg}$ $(2.52 \mathrm{mmol})$ of LiCl. Isolated yield 87\%. Found: $\mathrm{C} 44.88, \mathrm{H} 2.89, \mathrm{~N} 8.33$. Calcd. for $\mathrm{C}_{24} \mathrm{H}_{18} \mathrm{Cl}_{2} \mathrm{~N}_{4} \mathrm{Pd}_{2}: \mathrm{C} 44.61, \mathrm{H} 2.81, \mathrm{~N} 8.67$. b) $100.00 \mathrm{mg}(0.14 \mathrm{mmol})$ of $[(\mathrm{L} 1) \mathrm{Pd}(\mu-\mathrm{OAc})]_{2}(3)$ and $122.27 \mathrm{mg}(2.88 \mathrm{mmol})$ of $\mathrm{LiCl}$. Isolated yield 91\%. Found: $\mathrm{C} 44.88, \mathrm{H} 2.59, \mathrm{~N} \mathrm{8.82}$. c) $105.00 \mathrm{mg}(0.27 \mathrm{mmol})$ of $\{(\mathrm{L} 1) \mathrm{Pd}(\mathrm{acac})\}(7)$ and $115.09 \mathrm{mg}(2.71 \mathrm{mmol})$ of LiCl. Isolated yield $88 \%$. Found: C 44.28, H 2.49, N 8.78.

[(L2)Pd( $\boldsymbol{\mu}$-Cl) $]_{2}(\mathbf{6})$. a) $123.00 \mathrm{mg}(0.23 \mathrm{mmol})$ of $\left[(\mathrm{L} 2) \mathrm{Pd}(\mathrm{MeCN})_{2}\right]\left[\mathrm{BF}_{4}\right](\mathbf{2})$ and $97.63 \mathrm{mg}$ (2.30 mmol) of LiCl. Isolated yield 90\%. Found: C 42.09, H 2.96, N 10.19. Calcd. for $\mathrm{C}_{28} \mathrm{H}_{26} \mathrm{Cl}_{4} \mathrm{~N}_{6} \mathrm{Pd}_{2}: \mathrm{C} 41.97, \mathrm{H} 3.27, \mathrm{~N} 10.49$. b) $110.00 \mathrm{mg}(0.13 \mathrm{mmol})$ of $[(\mathrm{L} 2) \mathrm{Pd}(\mu-\mathrm{OAc})]_{2}$ (4) and $109.92 \mathrm{mg}(2.59 \mathrm{mmol})$ of LiCl. Isolated yield 86\%. Found: C 41.63, H 3.09, N 10.62. c) $115.00 \mathrm{mg}(0.25 \mathrm{mmol})$ of $\{(\mathrm{L} 2) \mathrm{Pd}(\mathrm{acac})\}(8)$ and $105.00 \mathrm{mg}(2.48 \mathrm{mmol})$ of $\mathrm{LiCl}$. Isolated yield 91\%. Found: C 41.58, H 3.49, N 10.73 .

$\left[\mathbf{P d}_{2}\left(\mathbf{H}_{2} \mathbf{O}\right)(\boldsymbol{\mu}-\mathbf{C l})(\boldsymbol{\mu}-\mathbf{L} \mathbf{1})(\mathbf{C l})\right]_{2}(\mathbf{9})$. a) $84.00 \mathrm{mg}(0.08 \mathrm{mmol})$ of $\left[\mathrm{Pd}(\mu-\mathrm{OAc})_{2}(\mu-\mathrm{L} 1) \mathrm{Pd}\right]_{2}(\mathbf{1 1})$ and $139,33 \mathrm{mg}(3.29 \mathrm{mmol})$ of $\mathrm{LiCl}$. Isolated yield $92 \%$. Found: C 38.03, H 2.33, N 7.14; Calcd. for $\mathrm{C}_{24} \mathrm{H}_{20} \mathrm{Cl}_{4} \mathrm{~N}_{4} \mathrm{O}_{2} \mathrm{Pd}_{2}: \mathrm{C} 38.38, \mathrm{H} 2.68, \mathrm{~N} 7.46$. b) $90.00 \mathrm{mg}(0.15 \mathrm{mmol})$ of $\{(\mathrm{acac}) \mathrm{Pd}(\mu-\mathrm{L} 1) \mathrm{Pd}(\mathrm{acac})\}(\mathbf{1 3})$ and $129.04(3.04 \mathrm{mmol})$ of $\mathrm{LiCl}$. Isolated yield 93\%. Found: C 38.49, H 2.74, N 7.23.

$\left[\mathbf{P d}_{2}\left(\mathbf{H}_{2} \mathbf{O}\right)(\boldsymbol{\mu}-\mathbf{C l})(\boldsymbol{\mu}-\mathbf{L} 2)(\mathbf{C l})\right]_{2}(\mathbf{1 0})$. a) $90.00 \mathrm{mg}(0.07 \mathrm{mmol})$ of $\left[\mathrm{Pd}(\mu-\mathrm{OAc})_{2}(\boldsymbol{\mu}-\mathrm{L} 2) \mathrm{Pd}\right]_{2}(\mathbf{1 2})$

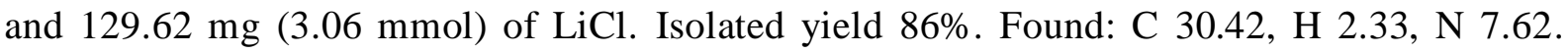
Calcd. for $\mathrm{C}_{28} \mathrm{H}_{28} \mathrm{Cl}_{6} \mathrm{~N}_{6} \mathrm{O}_{2} \mathrm{Pd}_{4}: \mathrm{C} 30.05, \mathrm{H} 2.52, \mathrm{~N} 7.51$. b) $98.00 \mathrm{mg}(0.14 \mathrm{mmol})$ of $\{(\mathrm{acac}) \mathrm{Pd}(\mu-\mathrm{L} 2) \mathrm{Pd}(\mathrm{acac}\}(\mathbf{1 4})$ and $124.23 \mathrm{mg}(2.93 \mathrm{mmol})$ of $\mathrm{LiCl}$. Isolated yield $94 \%$. Found: C 30.42, H 2.33, N 7.42. 


\section{NMR SPECTROSCOPY}

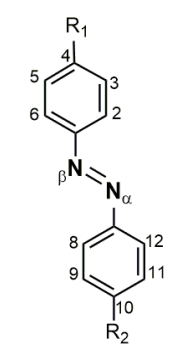

$\mathrm{R}_{1}=\mathrm{H} ; \mathrm{R}_{2}=\mathrm{H} A B$ $\mathrm{R}_{1}=\mathrm{N}\left(\mathrm{CH}_{3}\right)_{2} ; \mathrm{R}_{2}=\mathrm{Cl} \mathrm{DACl}$<smiles>[R6]c1ccc(N=Nc2ccc(Br)cc2[R16])cc1</smiles><smiles>[R8]c1ccc(N=Nc2ccc(Br)cc2)cc1</smiles>

Monopalladated complexes<smiles>[R6]c1ccc(N=Nc2ccc(Br)cc2C(=O)c2ccccc2)c(Br)c1</smiles>

Dipalladated complexes

Scheme S1. NMR numbering of protons in azobenzenes and their complexes.

\section{Table S1}

${ }^{1} \mathrm{H}$ NMR data $\left(\delta / \mathrm{ppm}, J / \mathrm{Hz}, \mathrm{CDCl}_{3}\right)$ for $\mathbf{L 1}, \mathbf{L 2}$ and their acetylacetonate complexes ${ }^{\mathrm{a}}$

\begin{tabular}{|c|c|c|c|c|c|c|c|c|}
\hline Proton & L1 & L2 & 7 & $7^{\mathrm{b}}$ & 8 & $8^{\text {b }}$ & 13 & 14 \\
\hline $\begin{array}{l}\mathrm{Me}(\text { acac }) \\
\mathrm{CH} \text { (acac) }\end{array}$ & - & & $\begin{array}{l}2.02 \mathrm{~s}, 3 \mathrm{H} \\
2.13 \mathrm{~s}, 3 \mathrm{H} \\
5.43 \mathrm{~s}, 1 \mathrm{H}\end{array}$ & $\begin{array}{l}2.00 \text { br s, } 3 \mathrm{H} \\
2.08, \text { br s, } 3 \mathrm{H} \\
5.55, \mathrm{~s}, 1 \mathrm{H}\end{array}$ & $\begin{array}{l}2.01 \mathrm{~s}, 3 \mathrm{H} \\
2.03 \mathrm{~s}, 3 \mathrm{H} \\
5.40 \mathrm{~s}, 1 \mathrm{H}\end{array}$ & $\begin{array}{l}1.98 \text { br s, } 3 \mathrm{H} \\
2.06, \text { br s, } 3 \mathrm{H} \\
5.50 \mathrm{~s}, 1 \mathrm{H}\end{array}$ & $\begin{array}{l}2.14 \mathrm{~s}, 6 \mathrm{H} \\
2.16 \mathrm{~s}, 6 \mathrm{H} \\
5.49 \mathrm{~s}, 2 \mathrm{H}\end{array}$ & $\begin{array}{l}2.09 \mathrm{~s}, 9 \mathrm{H} \\
2.13 \mathrm{~s}, 3 \mathrm{H} \\
5.43 \mathrm{~s}, 1 \mathrm{H} \\
5.44 \mathrm{~s}, 1 \mathrm{H}\end{array}$ \\
\hline $\mathrm{NMe}_{2}$ & - & $3.10 \mathrm{~s}, 6 \mathrm{H}$ & - & & $3.20 \mathrm{~s}, 6 \mathrm{H}$ & $3.19 \mathrm{~s}, 6 \mathrm{H}$ & & $3.20 \mathrm{~s}, 6 \mathrm{H}$ \\
\hline H-2 & $7.92 \mathrm{~d}, 1 \mathrm{H}$ & $\begin{array}{l}7.87 \mathrm{~d}, 1 \mathrm{H} \\
J=9.2\end{array}$ & - & & - & & - & - \\
\hline H-3 & $7.37-764, \mathrm{~m}$ & $\begin{array}{l}6.76 \mathrm{~d}, 1 \mathrm{H} \\
J=9.2\end{array}$ & $\begin{array}{l}7.61 \mathrm{~d}, 1 \mathrm{H} \\
J=7.3\end{array}$ & $\begin{array}{l}7.50, \mathrm{~d}, 1 \mathrm{H} \\
J=6.7\end{array}$ & $\begin{array}{l}6.80 \mathrm{ds}, 1 \mathrm{H} \\
J=2.6\end{array}$ & $\begin{array}{l}6.69 \mathrm{ds}, 1 \mathrm{H} \\
J=2.6\end{array}$ & $\begin{array}{l}7.67 \mathrm{dd}, 1 \mathrm{H} \\
J=7.4,1.6\end{array}$ & $\begin{array}{l}6.85 \mathrm{ds}, 1 \mathrm{H} \\
J=2.7\end{array}$ \\
\hline $\mathrm{H}-4$ & $7.37-764, \mathrm{~m}$ & & $\begin{array}{l}7.29 \mathrm{t}, 1 \mathrm{H} \\
J=7.3\end{array}$ & $7.32-7.39 \mathrm{~m}$ & - & - & $\begin{array}{l}7.20-7.32 \mathrm{~m} \text {, } \\
\text { overlapped } \\
\text { with } \mathrm{CDCl}_{3}\end{array}$ & - \\
\hline $\mathrm{H}-5$ & $7.37-764, \mathrm{~m}$ & $\begin{array}{l}6.76 \mathrm{~d}, 1 \mathrm{H} \\
J=9.2\end{array}$ & $\begin{array}{l}7.26 \mathrm{t}, 1 \mathrm{H} \\
J=7.4\end{array}$ & $7.32-7.39 \mathrm{~m}$ & $\begin{array}{l}6.49 \mathrm{dd}, 1 \mathrm{H} \\
J=8.8,2.6\end{array}$ & $\begin{array}{l}6.64 \mathrm{ds}, 1 \mathrm{H} \\
J=8.9,2.6\end{array}$ & $\begin{array}{l}7.20-7.32 \mathrm{~m} \text {, } \\
\text { overlapped } \\
\text { with } \mathrm{CDCl}_{3}\end{array}$ & $\begin{array}{l}6.45 \mathrm{dd}, 1 \mathrm{H} \\
J=9.3,2.7\end{array}$ \\
\hline H-6 & $7.92, \mathrm{~d}, 1 \mathrm{H}$ & $\begin{array}{l}7.87 \mathrm{~d}, 1 \mathrm{H} \\
J=9.2\end{array}$ & $\begin{array}{l}7.94 \mathrm{~d}, 1 \mathrm{H} \\
J=7.2\end{array}$ & $\begin{array}{l}8.00 \mathrm{~d}, 1 \mathrm{H} \\
J=6.7\end{array}$ & $\begin{array}{l}7.68 \mathrm{~d}, 1 \mathrm{H} \\
J=8.8\end{array}$ & $\begin{array}{l}7.66 \mathrm{~d}, 1 \mathrm{H} \\
J=8.9\end{array}$ & $\begin{array}{l}9.31 \mathrm{dd}, 1 \mathrm{H} \\
J=7.8,1.6\end{array}$ & $\begin{array}{l}9.05 \mathrm{~d}, 1 \mathrm{H} \\
J=9.3\end{array}$ \\
\hline $\mathrm{H}-8$ & $7.92, \mathrm{~d}, 1 \mathrm{H}$ & $\begin{array}{l}7.79 \mathrm{~d}, 1 \mathrm{H} \\
J=8.8\end{array}$ & $8.00-8.07 \mathrm{~m}$ & $7.94-7.98 \mathrm{~m}$ & $\begin{array}{l}7.36 \mathrm{~d}, 1 \mathrm{H} \\
J=9.0\end{array}$ & $\begin{array}{l}7.50, \mathrm{~d}, 1 \mathrm{H} \\
J=8.9\end{array}$ & - & - \\
\hline H-9 & 7.37-7 64, m & $\begin{array}{l}7.43 \mathrm{~d}, 1 \mathrm{H} \\
J=8.8\end{array}$ & $7.45-7.52 \mathrm{~m}$ & $7.55-7.62 \mathrm{~m}$ & $\begin{array}{l}7.92 \mathrm{~d}, 1 \mathrm{H} \\
J=8.9\end{array}$ & & $\begin{array}{l}7.67 \mathrm{dd}, 1 \mathrm{H} \\
J=7.4,1.6\end{array}$ & $\begin{array}{l}7.41 \mathrm{ds}, 1 \mathrm{H} \\
J=2.3\end{array}$ \\
\hline H-10 & 7.37-7 64, m & & $7.45-7.52 \mathrm{~m}$ & $7.55-7.62 \mathrm{~m}$ & - & & $\begin{array}{l}7.20-7.32 \mathrm{~m} \text {, } \\
\text { overlapped } \\
\text { with } \mathrm{CDCl}_{3} \\
\end{array}$ & - \\
\hline H-11 & 7.37-7 64, m & $\begin{array}{l}7.43 \mathrm{~d}, 1 \mathrm{H} \\
J=8.8\end{array}$ & $7.45-7.52 \mathrm{~m}$ & $7.55-7.62 \mathrm{~m}$ & $\begin{array}{l}7.92 \mathrm{~d}, 1 \mathrm{H} \\
J=8.9\end{array}$ & $\begin{array}{l}7.89 \mathrm{~d}, 1 \mathrm{H} \\
J=8.9\end{array}$ & $\begin{array}{l}7.20-7.32 \mathrm{~m} \\
\text { overlapped } \\
\text { with } \mathrm{CDCl}_{3}\end{array}$ & $\begin{array}{l}7.11 \mathrm{dd}, 1 \mathrm{H} \\
J=8.7,2.3\end{array}$ \\
\hline H-12 & $7.92, \mathrm{~d}, 1 \mathrm{H}$ & $\begin{array}{l}7.79 \mathrm{~d}, 1 \mathrm{H} \\
J=8.8\end{array}$ & $8.00-8.07 \mathrm{~m}$ & $7.94-7.98 \mathrm{~m}$ & $\begin{array}{l}7.36 \mathrm{~d}, 1 \mathrm{H} \\
J=9.0\end{array}$ & $\begin{array}{l}7.50, \mathrm{~d}, 1 \mathrm{H} \\
J=8.9\end{array}$ & $\begin{array}{l}9.31 \mathrm{dd}, 1 \mathrm{H} \\
J=7.8,1.6\end{array}$ & $\begin{array}{l}8.85 \mathrm{~d}, 1 \mathrm{H} \\
J=8.6\end{array}$ \\
\hline
\end{tabular}

${ }^{a} \mathrm{~s}$ - singlet, $\mathrm{d}$ - doublet, $\mathrm{t}$ - triplet, $\mathrm{m}$ - multiplet, br broad. ${ }^{\mathrm{b} S p e c t r a}$ of 7 and $\mathbf{8}$ recorded in DMSO- $d 6$ 
Table S2

${ }^{1} \mathrm{H}$ NMR data $\left(\delta / \mathrm{ppm}, J / \mathrm{Hz}, \mathrm{CDCl}_{3}\right)$ for $\mathbf{L 1}, \mathbf{L 2}$ and their acetate complexes ${ }^{\mathrm{a}}$

\begin{tabular}{|c|c|c|c|c|c|c|c|}
\hline Proton & L1 & L2 & $\mathbf{L 2}^{\mathrm{b}}$ & $3^{\mathrm{c}}$ & $4^{\mathrm{c}}$ & $11^{\mathrm{d}}$ & $12^{\mathrm{b}, \mathrm{d}}$ \\
\hline OAc & - & - & & $2.08 \mathrm{~s}, 3 \mathrm{H}$ & $2.1 \mathrm{~s}, 3 \mathrm{H}$ & $\begin{array}{l}2.19 \mathrm{~s}, 3 \mathrm{H} \\
2.20 \mathrm{~s}, 3 \mathrm{H}\end{array}$ & $\begin{array}{l}2.07 \mathrm{~s}, 3 \mathrm{H} \\
2.29 \mathrm{~s}, 3 \mathrm{H}\end{array}$ \\
\hline $\mathrm{NMe}_{2}$ & - & $3.10 \mathrm{~s}, 6 \mathrm{H}$ & $3.08 \mathrm{~s}, 6 \mathrm{H}$ & - & $2.97 \mathrm{~s}, 6 \mathrm{H}$ & - & $3.19 \mathrm{~s}, 6 \mathrm{H}$ \\
\hline $\mathrm{H}-2$ & $7.92, \mathrm{~d}, 1 \mathrm{H}$ & $\begin{array}{l}7.87 \mathrm{~d}, 1 \mathrm{H} \\
{ }^{3} J=9.2\end{array}$ & $\begin{array}{l}7.80 \mathrm{~d}, 2 \mathrm{H} \\
J=9.2\end{array}$ & - & - & - & - \\
\hline $\mathrm{H}-3$ & $\begin{array}{l}\text { 7.37-7.64, } \\
\mathrm{m}\end{array}$ & $\begin{array}{l}6.76 \mathrm{~d}, 1 \mathrm{H} \\
{ }^{3} J=9.2\end{array}$ & $\begin{array}{l}6.84 \mathrm{~d}, 2 \mathrm{H} \\
J=9.3\end{array}$ & $\begin{array}{l}6.49 \mathrm{dd}, 1 \mathrm{H} \\
{ }^{3} J=7.8,1.3\end{array}$ & $\begin{array}{l}5.59 \mathrm{ds}, 1 \mathrm{H} \\
J=2.6\end{array}$ & $6.68-6.88 \mathrm{~m}$ & $\begin{array}{l}5.87 \mathrm{ds}, 1 \mathrm{H} \\
J=2.4\end{array}$ \\
\hline $\mathrm{H}-4$ & $\begin{array}{l}\text { 7.37-7.64, } \\
\mathrm{m}\end{array}$ & - & - & $\begin{array}{l}6.80 \mathrm{dt}, 1 \mathrm{H} \\
{ }^{3} J=7.6,1.4\end{array}$ & - & $6.68-6.88 \mathrm{~m}$ & - \\
\hline $\mathrm{H}-5$ & $\begin{array}{l}\text { 7.37-7.64, } \\
\mathrm{m}\end{array}$ & $\begin{array}{l}6.76 \mathrm{~d}, 1 \mathrm{H} \\
{ }^{3} J=9.2\end{array}$ & $\begin{array}{l}6.84 \mathrm{~d}, 2 \mathrm{H} \\
J=9.3\end{array}$ & $\begin{array}{l}7.14 \mathrm{dt}, 1 \mathrm{H} \\
{ }^{3} J=7.5,1.1\end{array}$ & $\begin{array}{l}6.31 \mathrm{dd}, 1 \mathrm{H} \\
{ }^{3} J=8.9,2.6\end{array}$ & $6.68-6.88 \mathrm{~m}$ & $\begin{array}{l}6.29 \mathrm{dd}, 1 \mathrm{H} \\
J=9.4,2.4\end{array}$ \\
\hline H-6 & $7.92, \mathrm{~d}, 1 \mathrm{H}$ & $\begin{array}{l}7.87 \mathrm{~d}, 1 \mathrm{H} \\
{ }^{3} J=9.2\end{array}$ & $\begin{array}{l}7.80 \mathrm{~d}, 2 \mathrm{H} \\
J=9.2\end{array}$ & $\begin{array}{l}7.70 \mathrm{dd}, 1 \mathrm{H} \\
{ }^{3} J=7.7,1.4\end{array}$ & $\begin{array}{l}7.50 \mathrm{~d}, 1 \mathrm{H} \\
{ }^{3} J=8.9\end{array}$ & $\begin{array}{l}8.03, \mathrm{~d} 1 \mathrm{H} \\
J=8.2\end{array}$ & $\begin{array}{l}7.60 \mathrm{~d}, 1 \mathrm{H} \\
J=9.5\end{array}$ \\
\hline $\mathrm{H}-8$ & $7.92, \mathrm{~d}, 1 \mathrm{H}$ & $\begin{array}{l}7.79 \mathrm{~d}, 2 \mathrm{H} \\
{ }^{3} J=8.8\end{array}$ & $\begin{array}{l}7.78 \mathrm{~d}, 2 \mathrm{H} \\
J=8.8\end{array}$ & $\begin{array}{l}7.34, \mathrm{~d} 1 \mathrm{H} \\
{ }^{3} J=8.1\end{array}$ & $\begin{array}{l}7.03 \mathrm{~d}, 1 \mathrm{H} \\
{ }^{3} J=8.8\end{array}$ & - & - \\
\hline $\mathrm{H}-9$ & $\begin{array}{l}\text { 7.37-7.64, } \\
\mathrm{m}\end{array}$ & $\begin{array}{l}7.43 \mathrm{~d}, 1 \mathrm{H} \\
{ }^{3} J=8.8\end{array}$ & $\begin{array}{l}7.57 \mathrm{~d}, 2 \mathrm{H} \\
J=8.8\end{array}$ & $\begin{array}{l}7.25 \mathrm{t}, 1 \mathrm{H} \\
{ }^{3} \mathrm{~J}=7.9\end{array}$ & $\begin{array}{l}7.27 \mathrm{~d}, 1 \mathrm{H} \\
{ }^{3} J=8.8\end{array}$ & $6.68-6.88 \mathrm{~m}$ & $\begin{array}{l}6.46 \mathrm{ds}, 1 \mathrm{H} \\
J=2.1\end{array}$ \\
\hline $\mathrm{H}-10$ & $\begin{array}{l}\text { 7.37-7.64, } \\
\mathrm{m}\end{array}$ & - & - & $\begin{array}{l}7.37 \mathrm{t}, 1 \mathrm{H} \\
{ }^{3} J=7.9\end{array}$ & - & $6.68-6.88 \mathrm{~m}$ & - \\
\hline H-11 & $\begin{array}{l}\text { 7.37-7.64, } \\
\mathrm{m}\end{array}$ & $\begin{array}{l}7.43 \mathrm{~d}, 1 \mathrm{H} \\
{ }^{3} J=8.8\end{array}$ & $\begin{array}{l}7.57 \mathrm{~d}, 2 \mathrm{H} \\
J=8.8\end{array}$ & $\begin{array}{l}7.25 \mathrm{t}, 1 \mathrm{H} \\
{ }^{3} J=7.9\end{array}$ & $\begin{array}{l}7.27 \mathrm{~d}, 1 \mathrm{H} \\
{ }^{3} J=8.8\end{array}$ & $6.68-6.88 \mathrm{~m}$ & $\begin{array}{l}6.83 \mathrm{dd}, 1 \mathrm{H} \\
{ }^{3} J=8.6,2.2\end{array}$ \\
\hline $\mathrm{H}-12$ & $7.92, \mathrm{~d}, 1 \mathrm{H}$ & $\begin{array}{l}7.79 \mathrm{~d}, 2 \mathrm{H} \\
{ }^{3} J=8.8\end{array}$ & $\begin{array}{l}7.78 \mathrm{~d}, 2 \mathrm{H} \\
J=8.8\end{array}$ & $\begin{array}{l}7.34, \mathrm{~d} 1 \mathrm{H} \\
{ }^{3} J=8.1\end{array}$ & $\begin{array}{l}7.03 \mathrm{~d}, 1 \mathrm{H} \\
{ }^{3} J=8.8\end{array}$ & $\begin{array}{l}8.03, \mathrm{~d} 1 \mathrm{H} \\
J=8.2\end{array}$ & $\begin{array}{l}7.44 \mathrm{~d}, 1 \mathrm{H} \\
J=8.7\end{array}$ \\
\hline
\end{tabular}

${ }^{a} \mathrm{~s}$ - singlet, $\mathrm{d}$ - doublet, $\mathrm{t}$ - triplet, $\mathrm{m}$ - multiplet. ${ }^{\mathrm{b}}$ Spectra of $\mathbf{L 2}$ and $\mathbf{1 2}$ recorded in DMSO- $d 6$. ${ }^{\mathrm{c} D a t a}$ for $a n t i-$ transoid isomer. ${ }^{3}$ d Data for anti-cisoid isomer. ${ }^{3}$ 
Table S3

${ }^{1} \mathrm{H}$ NMR data $\left(\delta / \mathrm{ppm}, J / \mathrm{Hz}\right.$, DMSO- $\left.d_{6}\right)$ for L1, L2, and their chloride and tetrafluoroborate complexes $^{\text {a }}$

\begin{tabular}{|c|c|c|c|c|c|c|c|c|}
\hline Proton & L1 & L2 & $5^{\mathrm{b}}$ & $6^{\mathrm{b}}$ & 9 & 10 & 1 & $2^{\mathrm{b}}$ \\
\hline $\mathrm{NMe}_{2}$ & - & $3.08 \mathrm{~s}, 6 \mathrm{H}$ & - & $3.14 \mathrm{~s}, 6 \mathrm{H}$ & - & $3.19 \mathrm{~s}, 1 \mathrm{H}$ & - & $3.21 \mathrm{~s}, 6 \mathrm{H}$ \\
\hline $\mathrm{H}-2$ & $\begin{array}{l}7.89, \mathrm{~d}, 1 \mathrm{H} \\
J=6.9\end{array}$ & $\begin{array}{l}7.80 \mathrm{~d}, 1 \mathrm{H} \\
J=9.2\end{array}$ & - & - & - & - & - & - \\
\hline H-3 & $\begin{array}{l}7.60 \mathrm{t}, 1 \mathrm{H} \\
J=7.0\end{array}$ & $\begin{array}{l}6.84 \mathrm{~d}, 1 \mathrm{H} \\
J=9.3\end{array}$ & $\begin{array}{l}7.72 \mathrm{dd}, 1 \mathrm{H} \\
J=7.7,1.5\end{array}$ & $7.01 \mathrm{br} \mathrm{s}, 1 \mathrm{H}$ & $\begin{array}{l}8.75 \text { br d, } 1 \mathrm{H} \\
J=7.2\end{array}$ & $\begin{array}{l}7.24 \mathrm{ds}, 1 \mathrm{H} \\
J=2.4\end{array}$ & $7.25-7.45 \mathrm{~m}$ & $\begin{array}{l}6.63 \mathrm{ds}, 1 \mathrm{H} \\
J=2.3\end{array}$ \\
\hline $\mathrm{H}-4$ & $\begin{array}{l}7.58, \mathrm{t}, 1 \mathrm{H} \\
J=6.6\end{array}$ & - & $\begin{array}{l}7.28 \mathrm{dt}, 1 \mathrm{H} \\
J=7.4,1.4\end{array}$ & - & $7.18-7.28 \mathrm{~m}$ & - & $7.25-7.45 \mathrm{~m}$ & - \\
\hline H-5 & $\begin{array}{l}7.60 \mathrm{t}, 1 \mathrm{H} \\
J=7.0\end{array}$ & $\begin{array}{l}6.84 \mathrm{~d}, 1 \mathrm{H} \\
J=9.3\end{array}$ & $\begin{array}{l}7.21 \mathrm{dt}, 1 \mathrm{H} \\
J=7.6,1.6\end{array}$ & $\begin{array}{l}6.59 \mathrm{br} \mathrm{d}, 1 \mathrm{H} \\
J=8.6\end{array}$ & $7.18-7.28 \mathrm{~m}$ & $\begin{array}{l}6.65 \mathrm{dd}, 1 \mathrm{H} \\
J=9.5,2.6\end{array}$ & $7.25-7.45 \mathrm{~m}$ & $\begin{array}{l}6.68 \mathrm{dd}, 1 \mathrm{H} \\
J=9.0,2.3\end{array}$ \\
\hline H-6 & $\begin{array}{l}7.89, \mathrm{~d}, 1 \mathrm{H} \\
J=6.9\end{array}$ & $\begin{array}{l}7.80 \mathrm{~d}, 1 \mathrm{H} \\
J=9.2\end{array}$ & $\begin{array}{l}7.94 \mathrm{dd}, 1 \mathrm{H} \\
J=7.8,1.6\end{array}$ & $\begin{array}{l}7.44 \mathrm{~d}, 1 \mathrm{H} \\
J \approx 8.5\end{array}$ & $\begin{array}{l}7.87 \mathrm{~d}, 1 \mathrm{H} \\
J=7.1\end{array}$ & $\begin{array}{l}8.50 \mathrm{~d}, 1 \mathrm{H} \\
J=9.5\end{array}$ & $\begin{array}{l}8.04 \mathrm{~d}, 1 \mathrm{H} \\
J=7.4\end{array}$ & $\begin{array}{l}7.68 \mathrm{~d}, 1 \mathrm{H} \\
J=9.0\end{array}$ \\
\hline $\mathrm{H}-8$ & $\begin{array}{l}7.89, \mathrm{~d}, 1 \mathrm{H} \\
J=6.9\end{array}$ & $\begin{array}{l}7.78 \mathrm{~d}, 1 \mathrm{H} \\
J=8.8\end{array}$ & $7.74-7.82 \mathrm{~m}$ & $\begin{array}{l}7.66 \mathrm{~d}, 1 \mathrm{H} \\
J=8.5\end{array}$ & - & - & $7.72-7.82 \mathrm{~m}$ & $\begin{array}{l}7.58 \mathrm{~d}, 1 \mathrm{H} \\
J=8.7\end{array}$ \\
\hline H-9 & $\begin{array}{l}7.60 \mathrm{t}, 1 \mathrm{H} \\
J=7.0\end{array}$ & $\begin{array}{l}7.57 \mathrm{~d}, 1 \mathrm{H} \\
J=8.8\end{array}$ & $7.47-7.63 \mathrm{~m}$ & $\begin{array}{l}7.46 \mathrm{~d}, 1 \mathrm{H} \\
J=8.8\end{array}$ & $\begin{array}{l}8.75 \text { br d, } 1 \mathrm{H} \\
J=7.2\end{array}$ & $\begin{array}{l}7.63 \mathrm{ds}, 1 \mathrm{H} \\
J=2.3\end{array}$ & $7.55-7.68 \mathrm{~m}$ & $\begin{array}{l}7.63 \mathrm{~d}, 1 \mathrm{H} \\
J=8.7\end{array}$ \\
\hline $\mathrm{H}-10$ & $\begin{array}{l}7.58, \mathrm{t}, 1 \mathrm{H} \\
{ }^{3} J=6.6\end{array}$ & - & $7.47-7.63 \mathrm{~m}$ & - & $7.18-7.28 \mathrm{~m}$ & - & $7.55-7.68 \mathrm{~m}$ & - \\
\hline H-11 & $\begin{array}{l}7.60 \mathrm{t}, 1 \mathrm{H} \\
{ }^{3} J=7.0\end{array}$ & $\begin{array}{l}7.57 \mathrm{~d}, 1 \mathrm{H} \\
J=8.8\end{array}$ & $7.47-7.63 \mathrm{~m}$ & $\begin{array}{l}7.46 \mathrm{~d}, 1 \mathrm{H} \\
J=8.5\end{array}$ & $7.18-7.28 \mathrm{~m}$ & $\begin{array}{l}7.13 \mathrm{dd}, 1 \mathrm{H} \\
J=8.5,2.4\end{array}$ & $7.55-7.68 \mathrm{~m}$ & $\begin{array}{l}7.63 \mathrm{~d}, 1 \mathrm{H} \\
J=8.7\end{array}$ \\
\hline H-12 & $\begin{array}{l}7.89, \mathrm{~d}, 1 \mathrm{H} \\
{ }^{3} J=6.9\end{array}$ & $\begin{array}{l}7.78 \mathrm{~d}, 1 \mathrm{H} \\
J=8.8\end{array}$ & $7.74-7.82 \mathrm{~m}$ & $\begin{array}{l}7.66 \mathrm{~d}, 1 \mathrm{H} \\
J=8.5\end{array}$ & $\begin{array}{l}8.75 \text { br d, } 1 \mathrm{H} \\
J=7.2\end{array}$ & $\begin{array}{l}8.28 \mathrm{~d}, 1 \mathrm{H} \\
J=8.7\end{array}$ & $7.72-7.82 \mathrm{~m}$ & $\begin{array}{l}7.58 \mathrm{~d}, 1 \mathrm{H} \\
J=8.7\end{array}$ \\
\hline
\end{tabular}

${ }^{\mathrm{a}} \mathrm{S}-$ singlet, $\mathrm{d}$ - doublet, $\mathrm{t}$ - triplet, $\mathrm{m}$ - multiplet, br - broad. ${ }^{\mathrm{b}}$ Data for alpha isomer. 


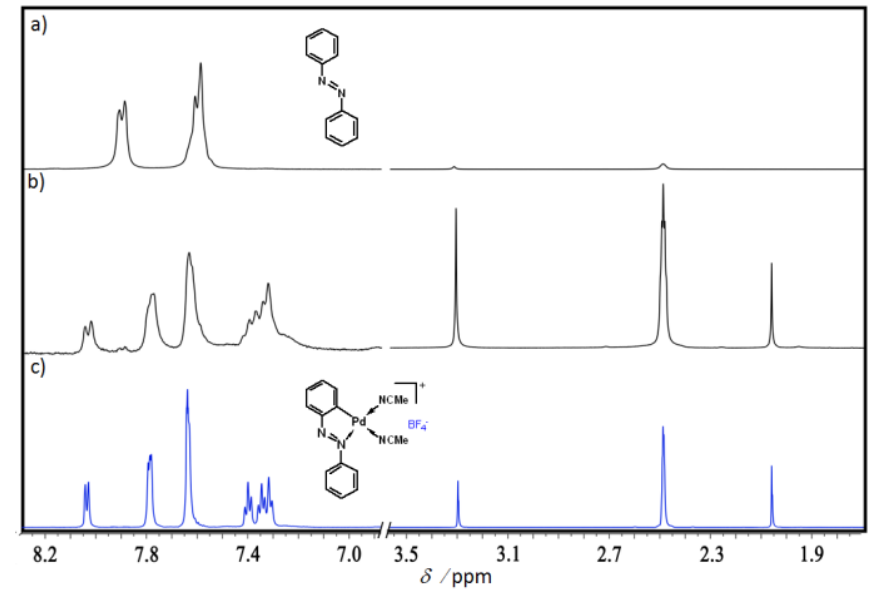

Figure S1. ${ }^{1} \mathrm{H}-\mathrm{NMR}\left(\mathrm{DMSO}-d_{\sigma}\right)$ spectra of: a) L1, b) crude product 1 prepared by IAG using $\mathrm{NaOAc}$ and $\mathrm{NaBF}_{4}$ as additives ${ }^{1}$ c) pure product $\mathbf{1}$ after washing with water.

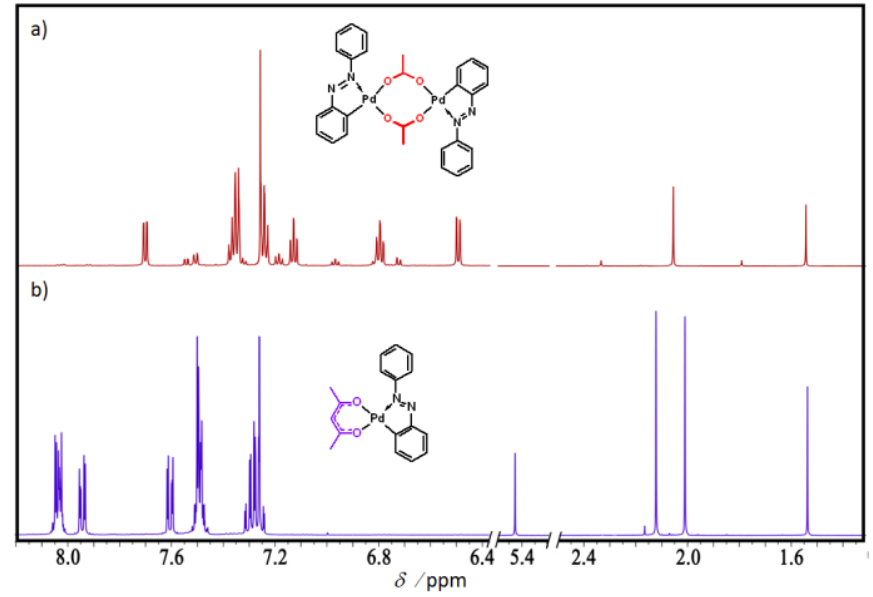

Figure S3. ${ }^{1} \mathrm{H}-\mathrm{NMR}\left(\mathrm{CDCl}_{3}\right)$ spectra of: a) precursor 3 and b) product 7 obtained from $\mathbf{3}$ by anion substitution using $\operatorname{Li}(\mathrm{acac})$.

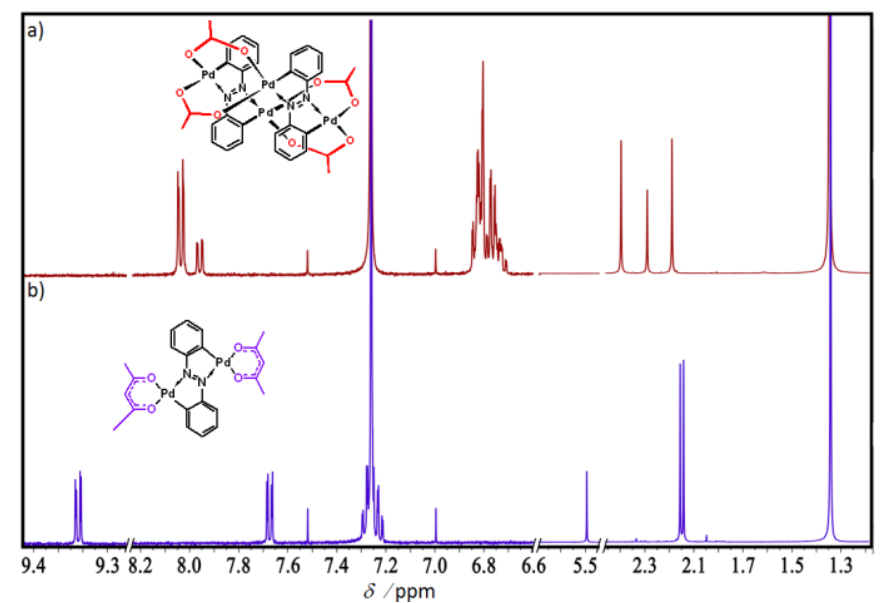

Figure S5. ${ }^{1} \mathrm{H}-\mathrm{NMR}\left(\mathrm{CDCl}_{3}\right)$ spectra of: a) precursor 11 and $\mathrm{b})$ product $\mathbf{1 3}$ obtained from $\mathbf{1 1}$ by anion substitution using Li(acac).

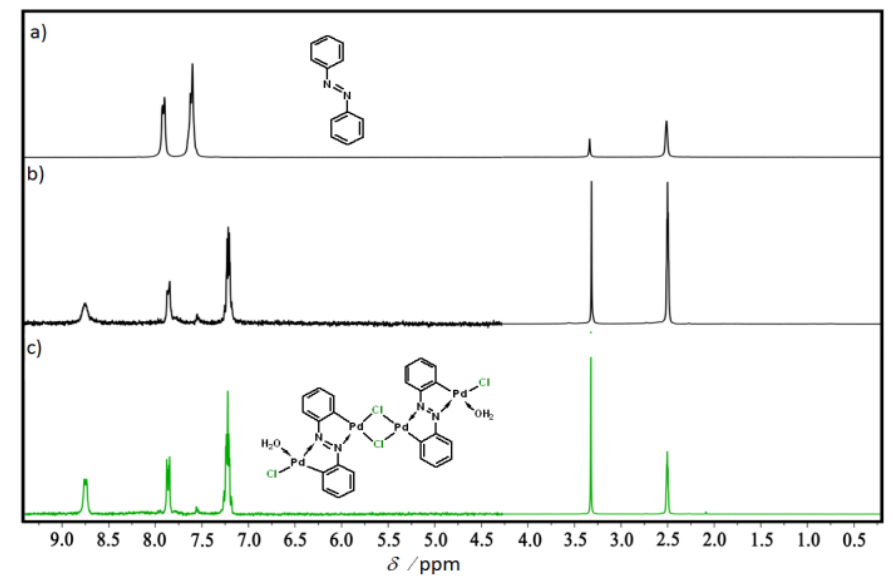

Figure S2. ${ }^{1} \mathrm{H}-\mathrm{NMR}\left(\mathrm{DMSO}-d_{6}\right)$ spectra of: a) L1, b) 9 prepared by ILAG using $\mathrm{NaOAc}$ and $\mathrm{H}_{2} \mathrm{O}$ as additives ${ }^{1}$ and c) pure product 9 after washing with water.

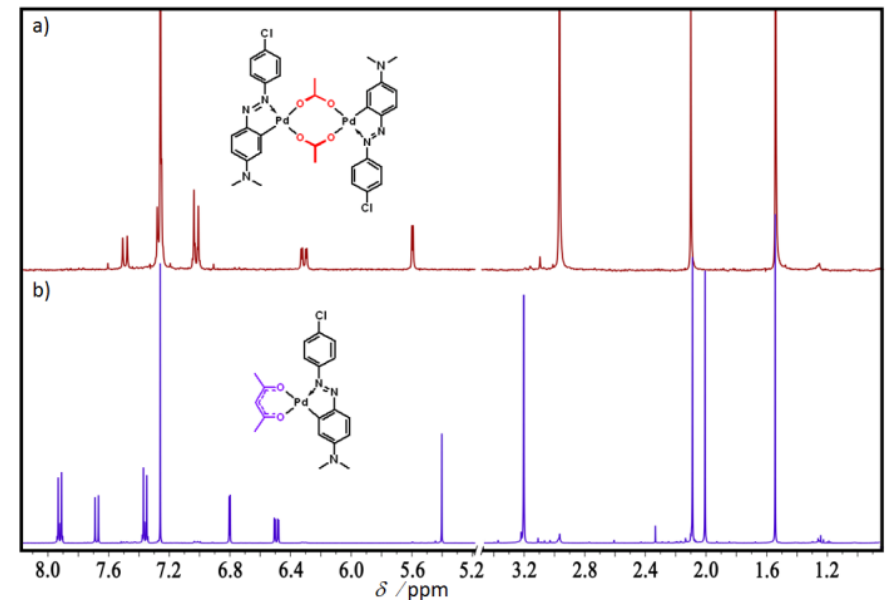

Figure S4. ${ }^{1} \mathrm{H}-\mathrm{NMR}\left(\mathrm{CDCl}_{3}\right)$ spectra of: a) 4 and b) product 8 obtained from 4 by anion substitution using Li(acac).

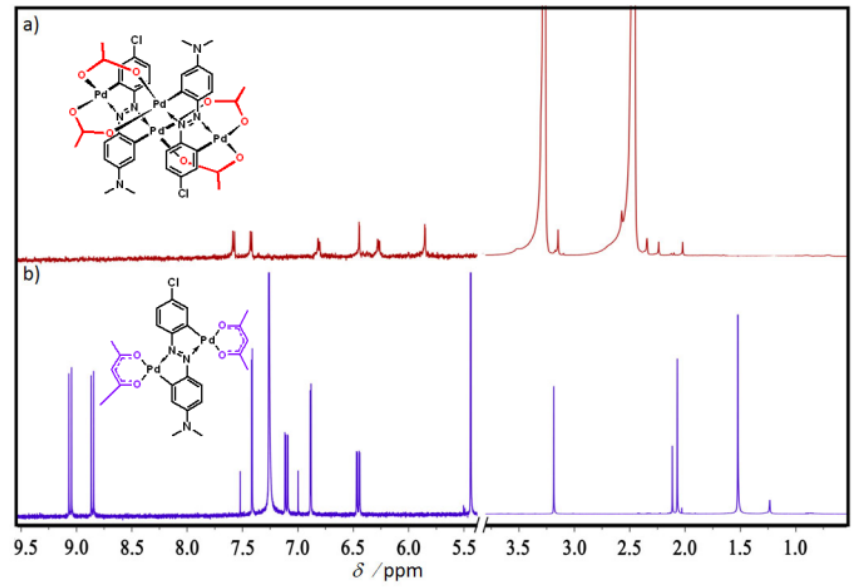

Figure S6. ${ }^{1} \mathrm{H}-\mathrm{NMR}$ spectra of: a) precursor 12 (DMSO- $d_{6}$ ) and b) product $14\left(\mathrm{CDCl}_{3}\right)$ obtained from 12 by anion substitution using $\operatorname{Li}(\mathrm{acac})$. 


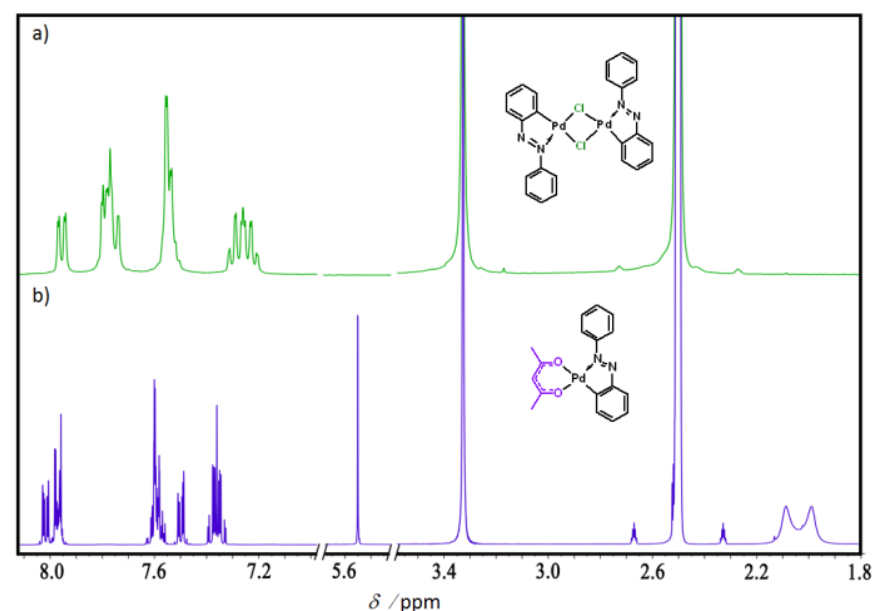

Figure S7. ${ }^{1} \mathrm{H}-\mathrm{NMR}$ (DMSO- $d_{6}$ ) spectra of: a) precursor $\mathbf{5}$ and b) product $\mathbf{7}$ obtained from $\mathbf{5}$ by anion substitution using $\mathrm{Li}(\mathrm{acac})$.

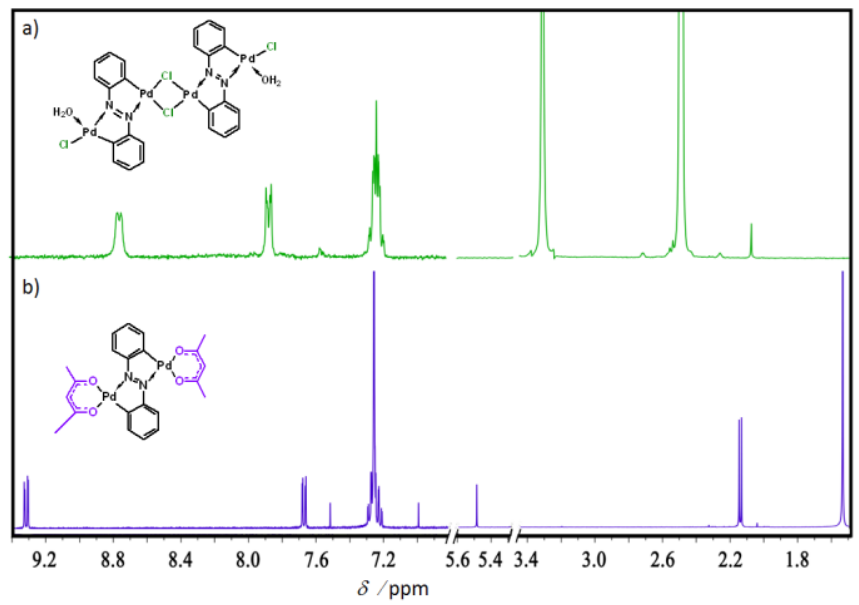

Figure S9. ${ }^{1} \mathrm{H}-\mathrm{NMR}$ spectra of: a) precursor 9 (DMSO$\left.\mathrm{d}_{6}\right)$ and b) product $\mathbf{1 3}\left(\mathrm{CDCl}_{3}\right)$ obtained from 9 anion substitution using $\mathrm{Li}(\mathrm{acac})$.

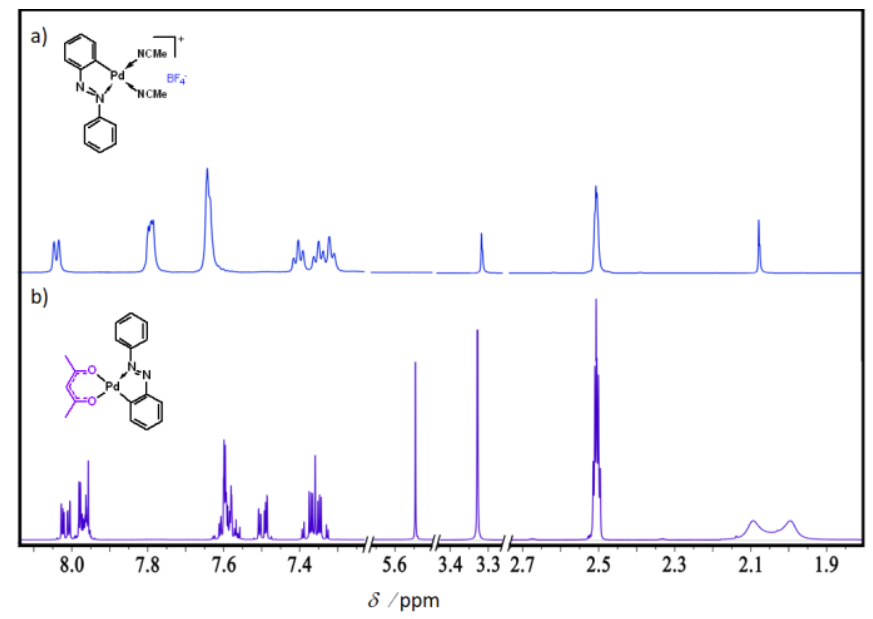

Figure S11. ${ }^{1} \mathrm{H}-\mathrm{NMR}$ (DMSO-d6) spectra of: a) precursor $\mathbf{1}$ and b) product $\mathbf{7}$ obtained from a) by anion substitution using $\mathrm{Li}(\mathrm{acac})$.

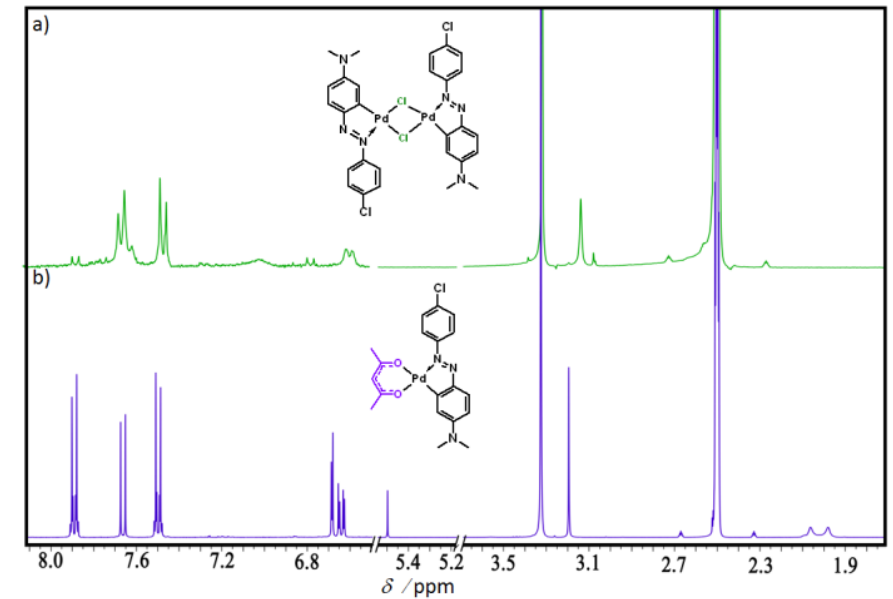

Figure S8. ${ }^{1} \mathrm{H}-\mathrm{NMR}\left(\mathrm{DMSO}-d_{6}\right)$ spectra of: a) precursor 6 and b) product $\mathbf{8}$ obtained from $\mathbf{6}$ by anion substitution using $\mathrm{Li}(\mathrm{acac})$.

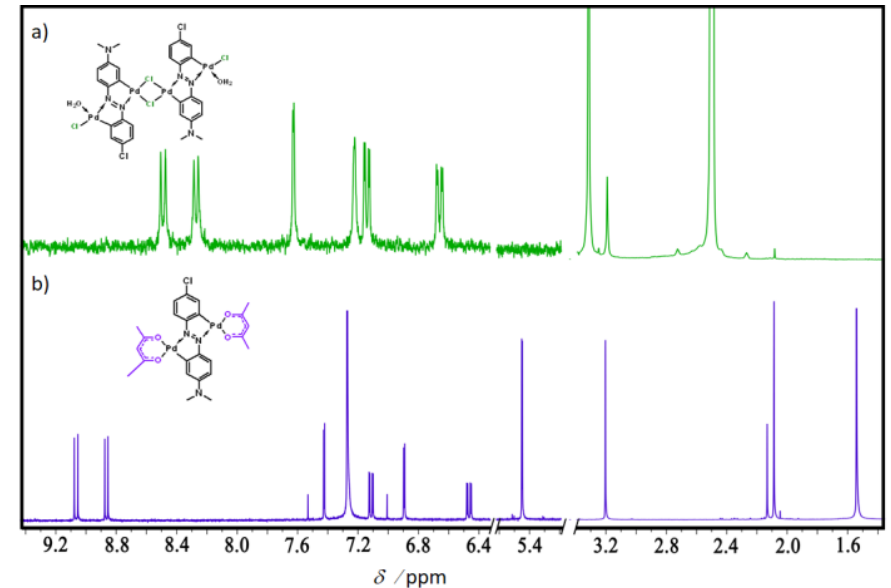

Figure S10. ${ }^{1} \mathrm{H}-\mathrm{NMR}$ (DMSO-d6) spectra of: a) precursor 10 and b) product $14\left(\mathrm{CDCl}_{3}\right)$ obtained from $\mathbf{1 0}$ by anion substitution using $\mathrm{Li}(\mathrm{acac})$.

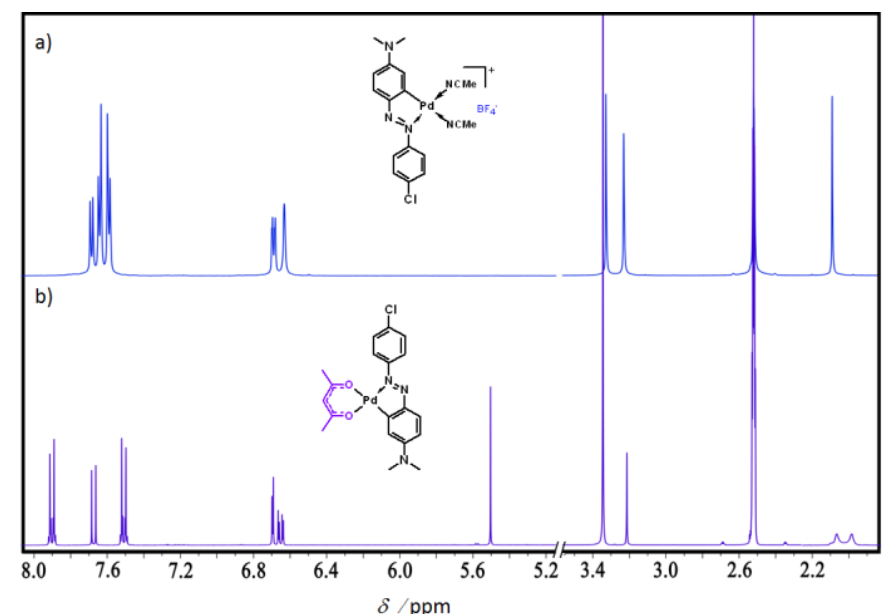

Figure S12. ${ }^{1} \mathrm{H}-\mathrm{NMR}$ (DMSO-d ${ }_{6}$ ) spectra of: a) precursor 2 and b) product $\mathbf{8}$ obtained from $\mathbf{2}$ by anion substitution using Li(acac). 


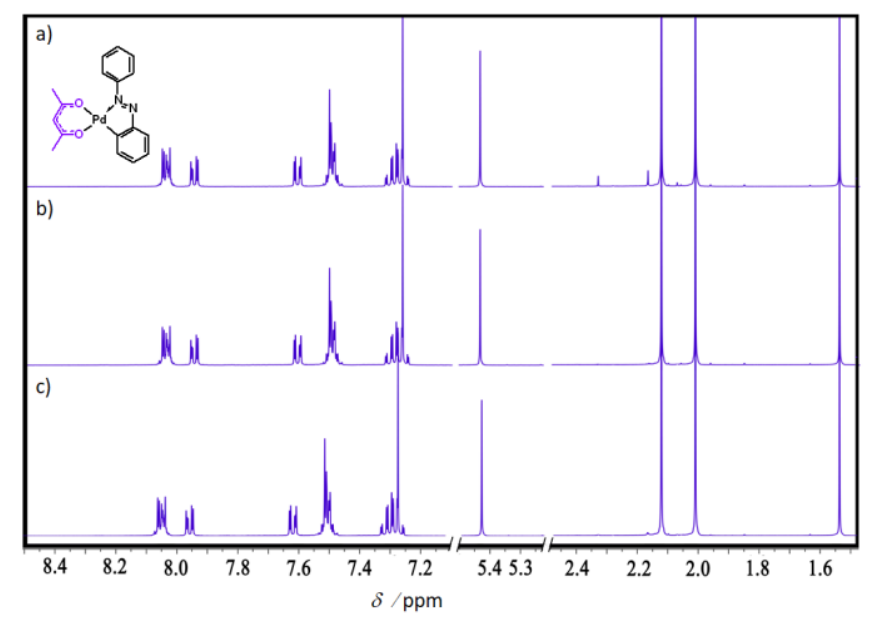

Figure S13. ${ }^{1} \mathrm{H}-\mathrm{NMR}\left(\mathrm{CDCl}_{3}\right)$ spectra of product 7 obtained by anion substitution using $\mathrm{Li}(\mathrm{acac})$ from precursors: a) 1, b) $\mathbf{3}$, and c) $\mathbf{5}$.

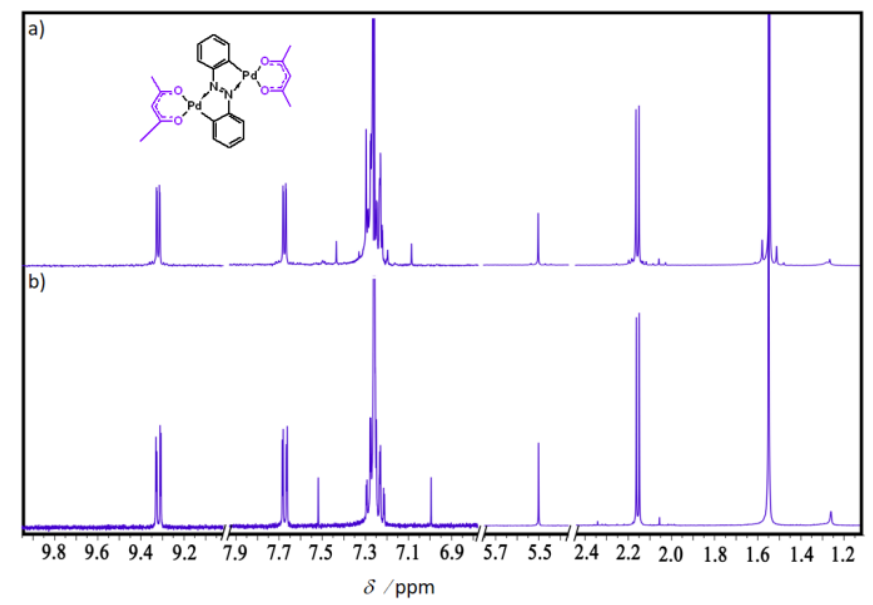

Figure S15. ${ }^{1} \mathrm{H}-\mathrm{NMR}\left(\mathrm{CDCl}_{3}\right)$ spectra of product 13 obtained by anion substitution using $\mathrm{Li}(\mathrm{acac})$ from precursors: a) 9 and b) 11.

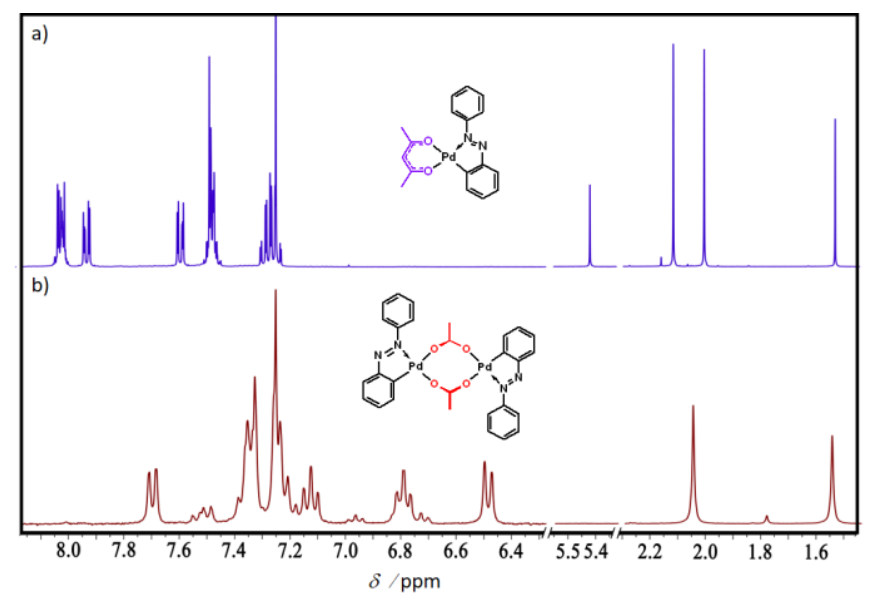

Figure S17. ${ }^{1} \mathrm{H}-\mathrm{NMR}\left(\mathrm{CDCl}_{3}\right)$ spectra of: a) precursor 7 and b) product $\mathbf{3}$ obtained from $\mathbf{7}$ by anion substitution using $\mathrm{NaOAc}$.

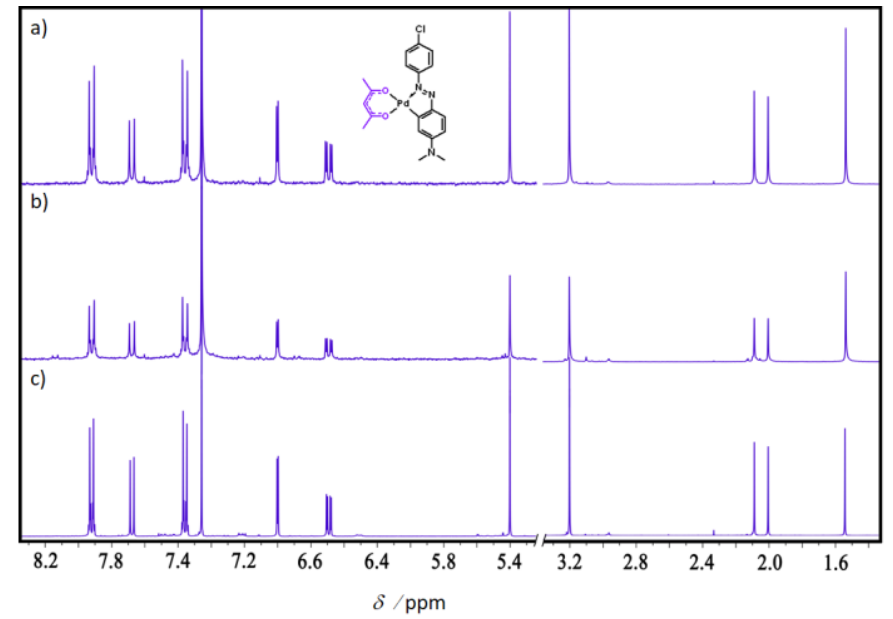

Figure S14. ${ }^{1} \mathrm{H}-\mathrm{NMR}\left(\mathrm{CDCl}_{3}\right)$ spectra of product 8 obtained by anion substitution using $\operatorname{Li}(\mathrm{acac})$ from precursors: a) 2, b) 4 and c) 6 .

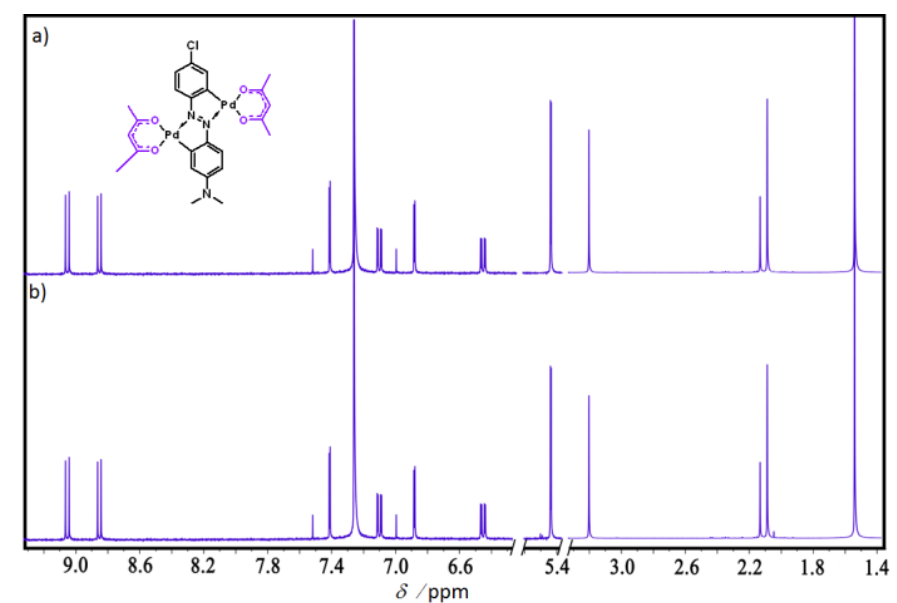

Figure S16. ${ }^{1} \mathrm{H}-\mathrm{NMR}\left(\mathrm{CDCl}_{3}\right)$ spectra of product 14 obtained by anion substitution using $\mathrm{Li}(\mathrm{acac})$ from precursors: a) $\mathbf{1 0}$ and b) 12 .

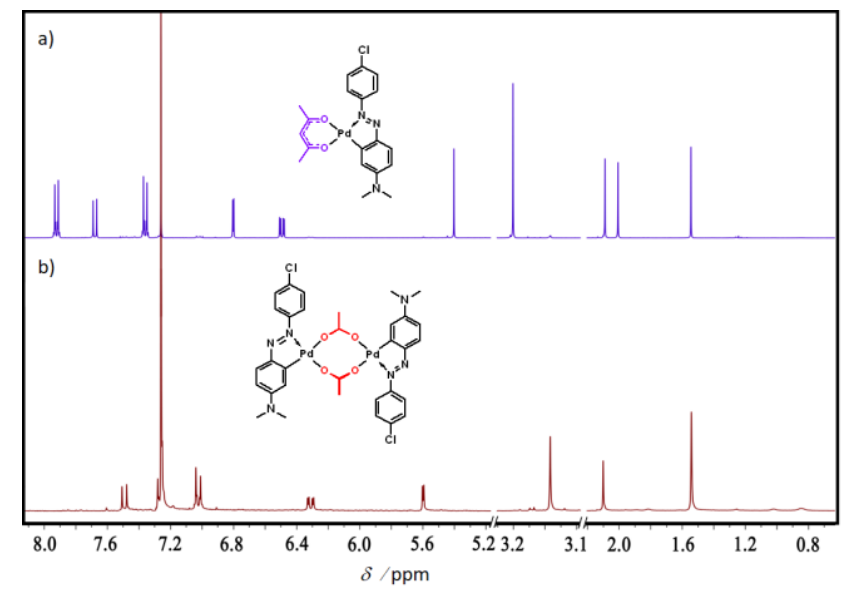

Figure S18. ${ }^{1} \mathrm{H}-\mathrm{NMR}\left(\mathrm{CDCl}_{3}\right)$ spectra of: a) precursor 8 and b) product 4 obtained from 8 by anion substitution using $\mathrm{NaOAc}$. 


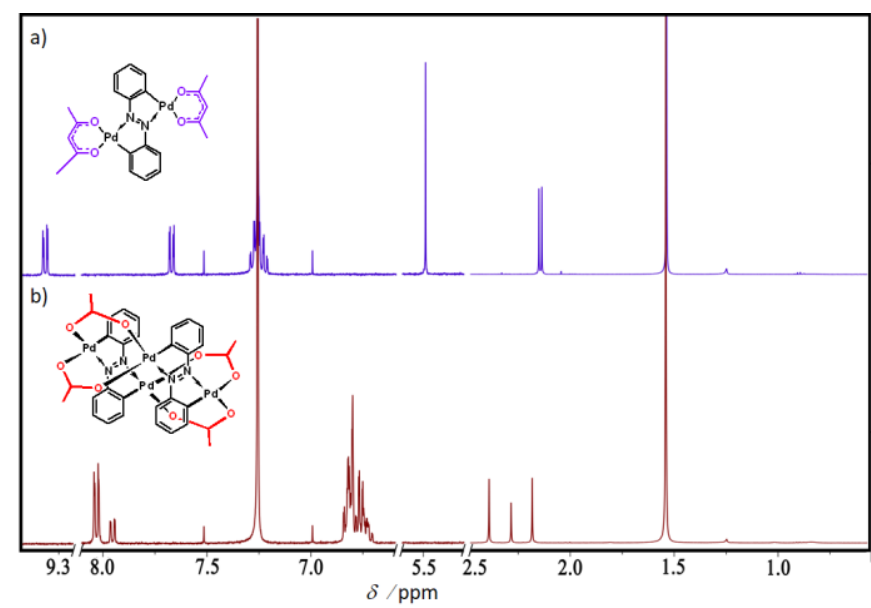

Figure S19. ${ }^{1} \mathrm{H}-\mathrm{NMR}\left(\mathrm{CDCl}_{3}\right)$ spectra of a) precursor 13 and b) product 11 obtained from 13 by anion substitution using $\mathrm{NaOAc}$.

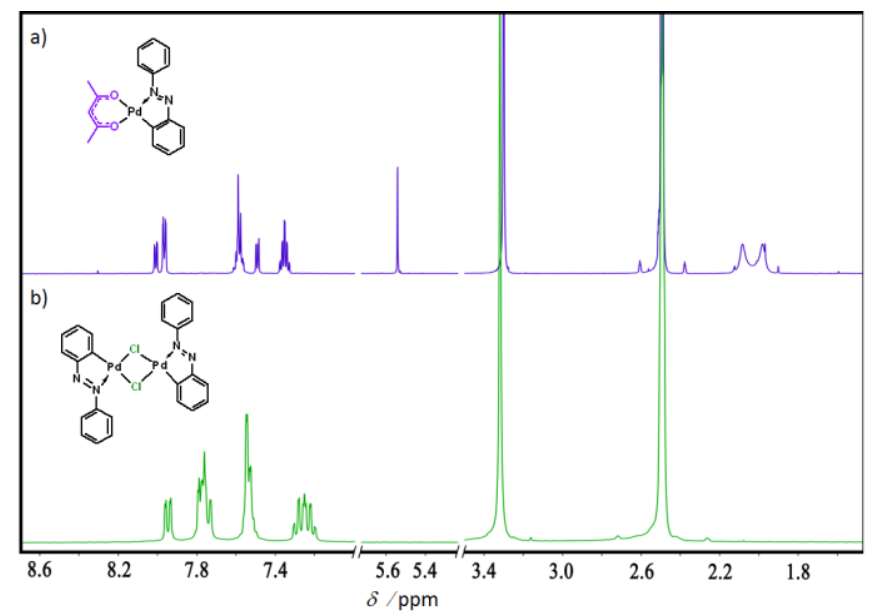

Figure S21. ${ }^{1} \mathrm{H}-\mathrm{NMR}$ (DMSO-d 6 ) spectra of a) precursor 7 and b) product 5 obtained from $\mathbf{7}$ by anion substitution using $\mathrm{LiCl}$.

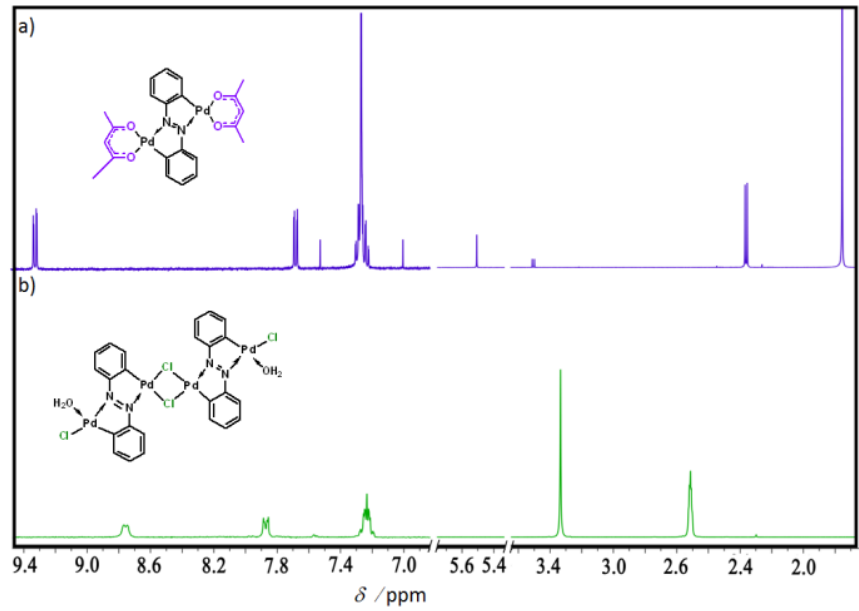

Figure S23. ${ }^{1} \mathrm{H}-\mathrm{NMR}$ spectra of a) precursor $\mathbf{1 3}$ $\left(\mathrm{CDCl}_{3}\right)$ and b) product 9 (DMSO-d6) obtained from 13 by anion substitution using $\mathrm{LiCl}$.

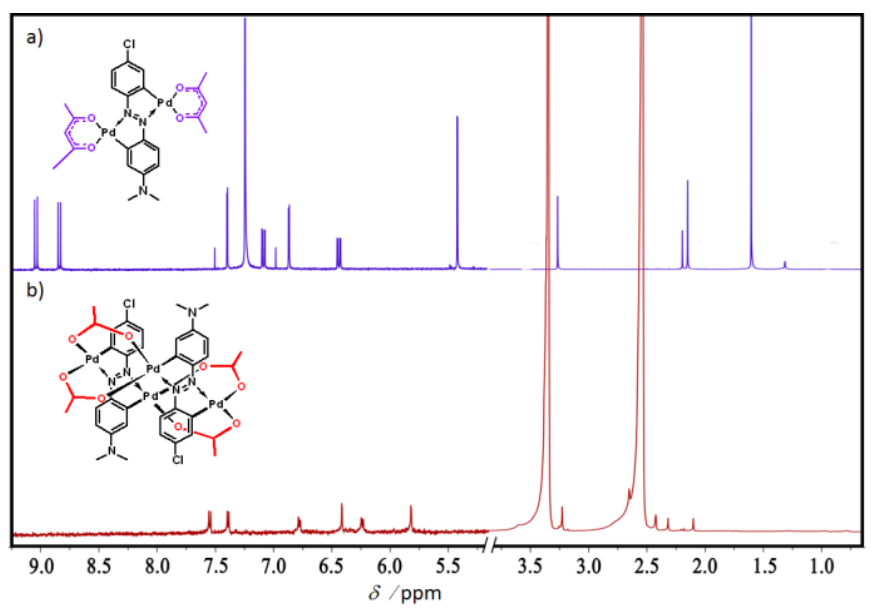

Figure S20. ${ }^{1} \mathrm{H}-\mathrm{NMR}$ (DMSO-d6) spectra of a) precursor 14 and b) product 12 obtained from $\mathbf{1 4}$ by anion substitution using $\mathrm{NaOAc}$.

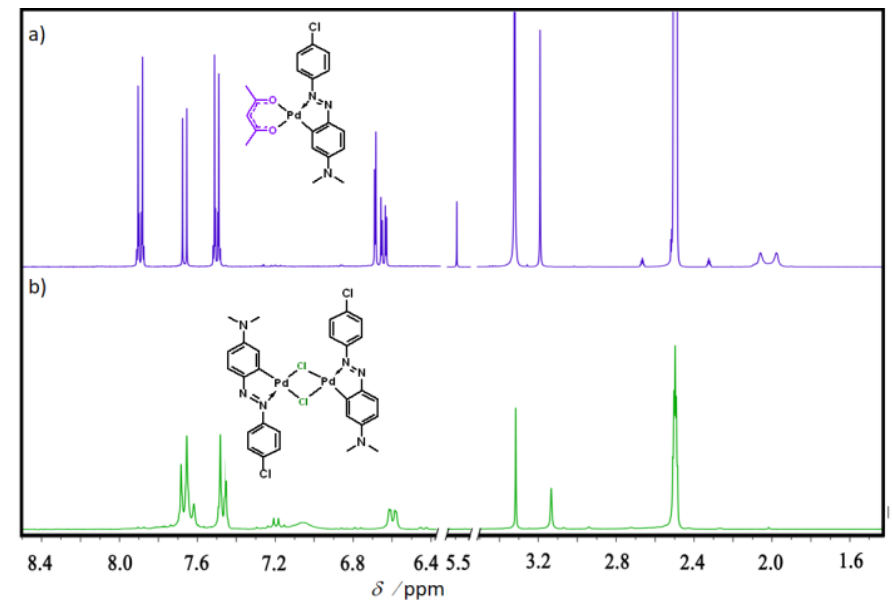

Figure S22. ${ }^{1} \mathrm{H}-\mathrm{NMR}$ (DMSO-d6) spectra of a) precursor 8 and b) product $\mathbf{6}$ obtained from $\mathbf{8}$ by anion substitution using $\mathrm{LiCl}$.

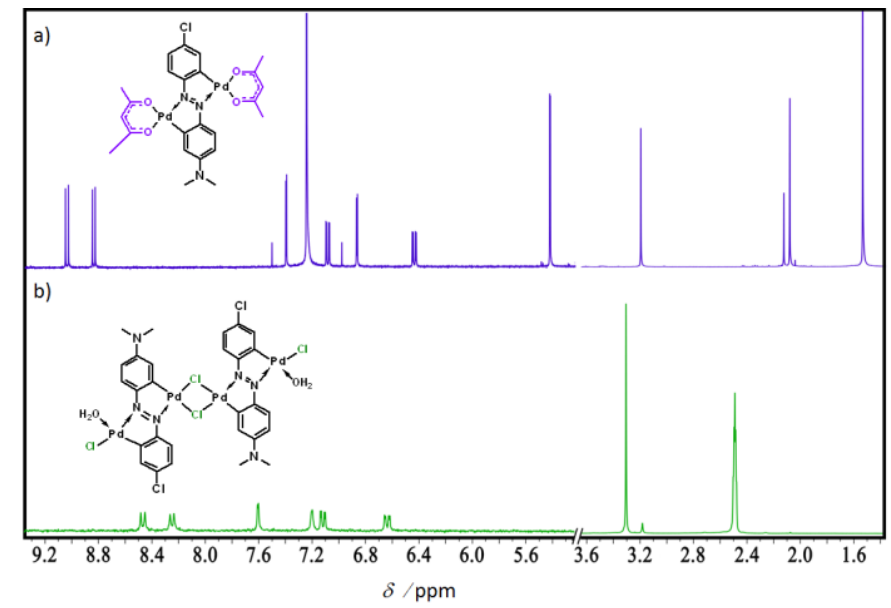

Figure S24. ${ }^{1} \mathrm{H}-\mathrm{NMR}$ spectra of a) precursor $14\left(\mathrm{CDCl}_{3}\right)$ and b) product 10 (DMSO-d $\mathrm{d}_{6}$ ) obtained from $\mathbf{1 4}$ by anion substitution using $\mathrm{LiCl}$. 


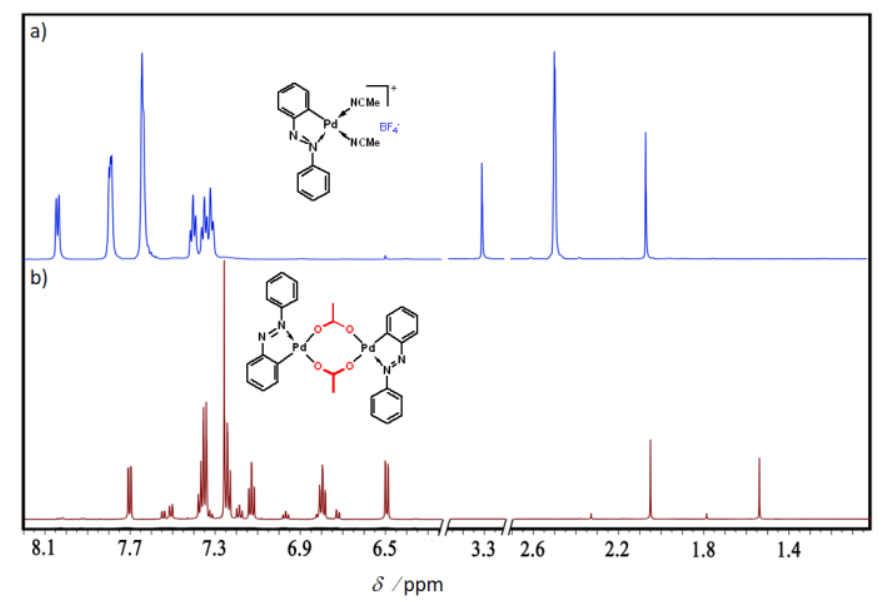

Figure S25. ${ }^{1} \mathrm{H}-\mathrm{NMR}$ spectra of: a) precursor $\mathbf{1}$ (DMSO-d6) and b) product $3\left(\mathrm{CDCl}_{3}\right)$ obtained from $\mathbf{1}$ by anion substitution using $\mathrm{NaOAc}$.

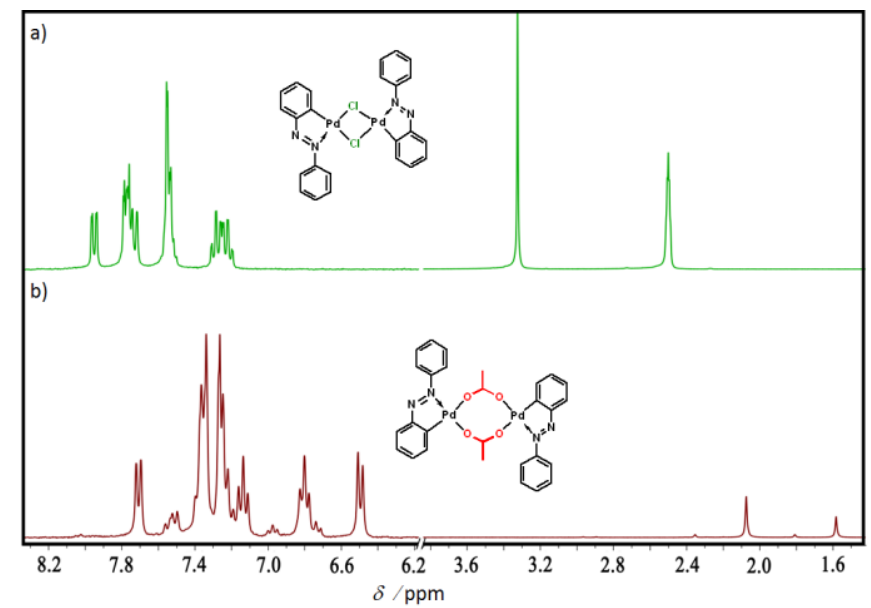

Figure S27. ${ }^{1} \mathrm{H}-\mathrm{NMR}$ (DMSO-d 6 ) spectra of a) precursor 5 and b) product $\mathbf{3}$ obtained from $5\left(\mathrm{CDCl}_{3}\right)$ by anion substitution using $\mathrm{NaOAc}$.

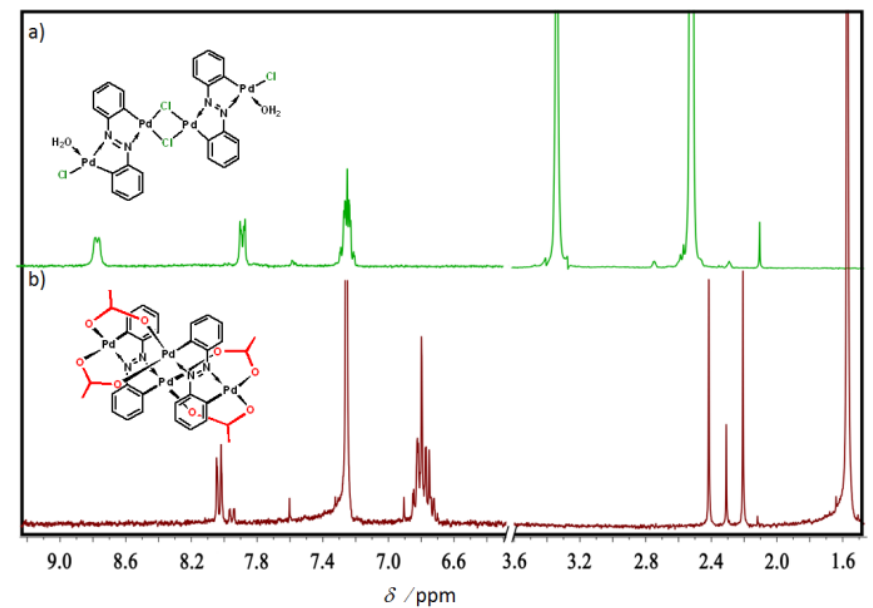

Figure S29. ${ }^{1} \mathrm{H}-\mathrm{NMR}$ spectra of a) precursor 9 (DMSO$\left.\mathrm{d}_{6}\right)$ and b) product $11\left(\mathrm{CDCl}_{3}\right)$ obtained from 9 by anion substitution using $\mathrm{NaOAc}$.

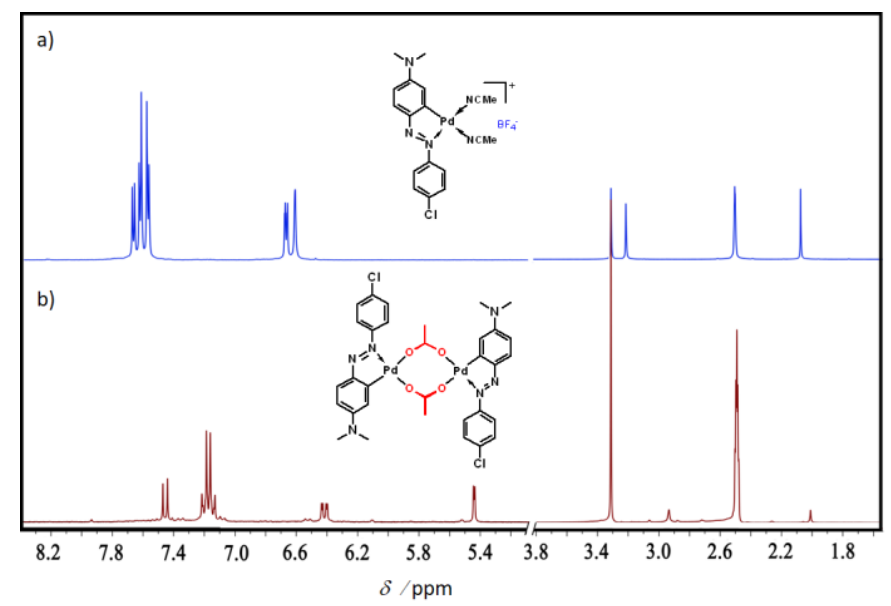

Figure S26. ${ }^{1} \mathrm{H}-\mathrm{NMR}$ (DMSO-d6) spectra of: a) precursor 2 and b) product $\mathbf{4}$ obtained from $\mathbf{2}$ by anion substitution using $\mathrm{NaOAc}$.

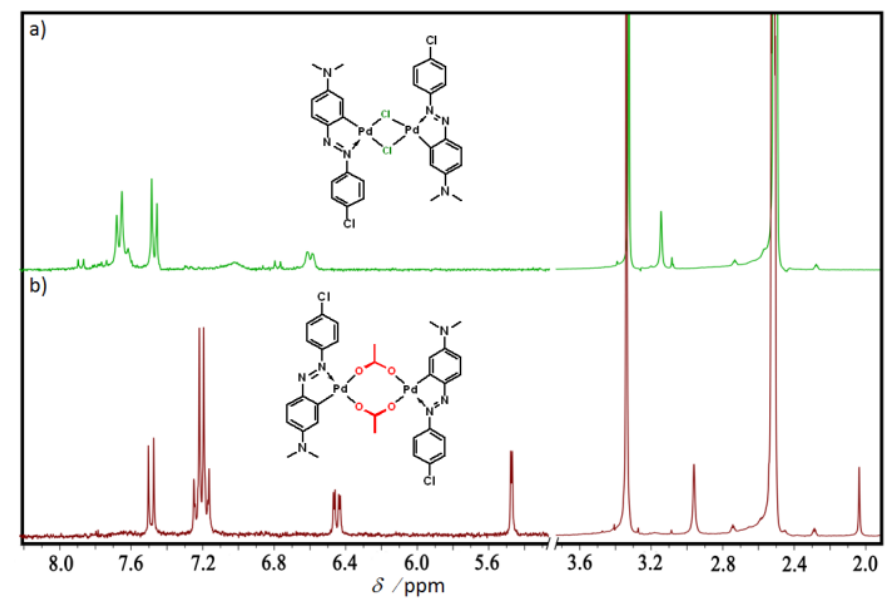

Figure S28. ${ }^{1} \mathrm{H}-\mathrm{NMR}$ (DMSO-d6) spectra of a) precursor 6 and b) product 4 obtained from $\mathbf{6}$ by anion substitution using $\mathrm{NaOAc}$.

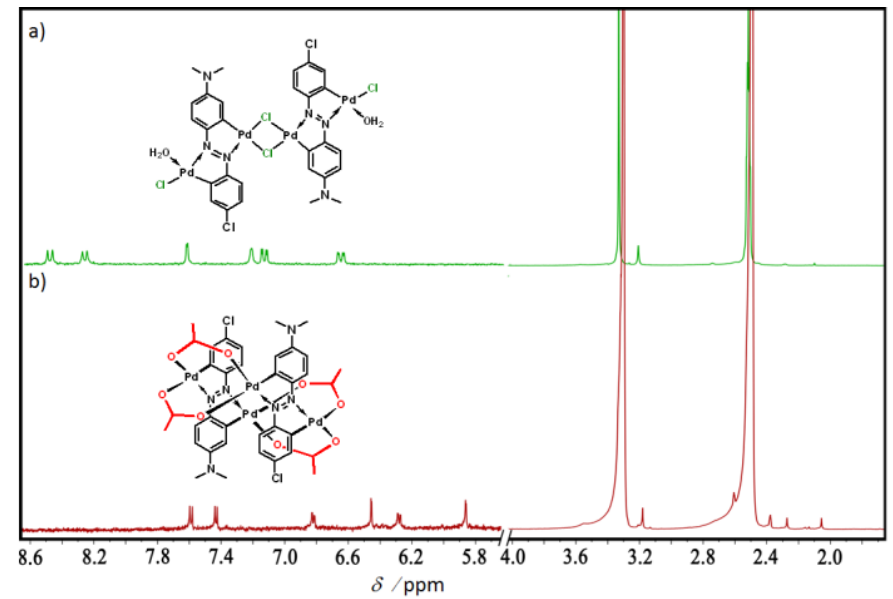

Figure S30. ${ }^{1} \mathrm{H}-\mathrm{NMR}$ (DMSO-d6) spectra of a) precursor 10 and b) product $\mathbf{1 2}$ obtained from $\mathbf{1 0}$ by anion substitution using NaOAc. 


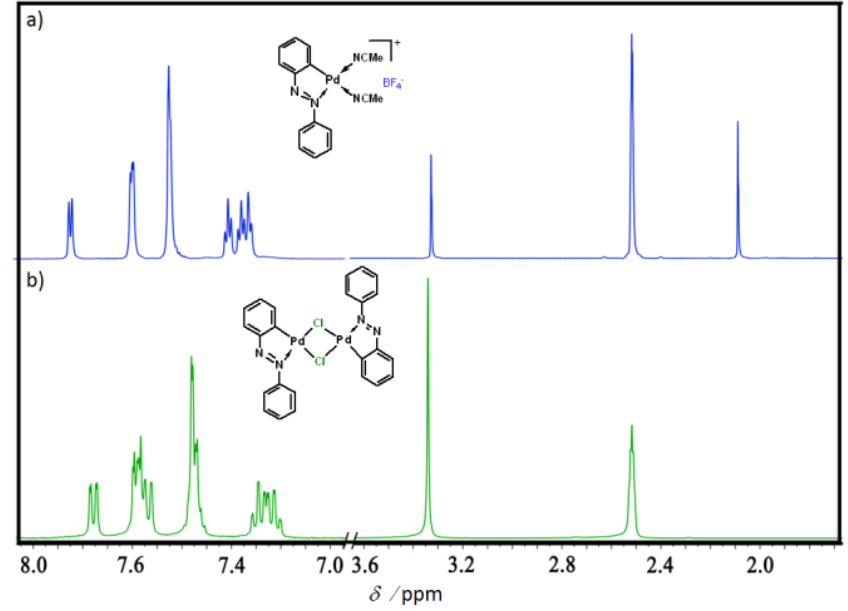

Figure S31. ${ }^{1} \mathrm{H}-\mathrm{NMR}$ (DMSO-d6) spectra of a) precursor $\mathbf{1}$ and b) product $\mathbf{5}$ obtained from $\mathbf{1}$ by anion substitution using $\mathrm{LiCl}$.

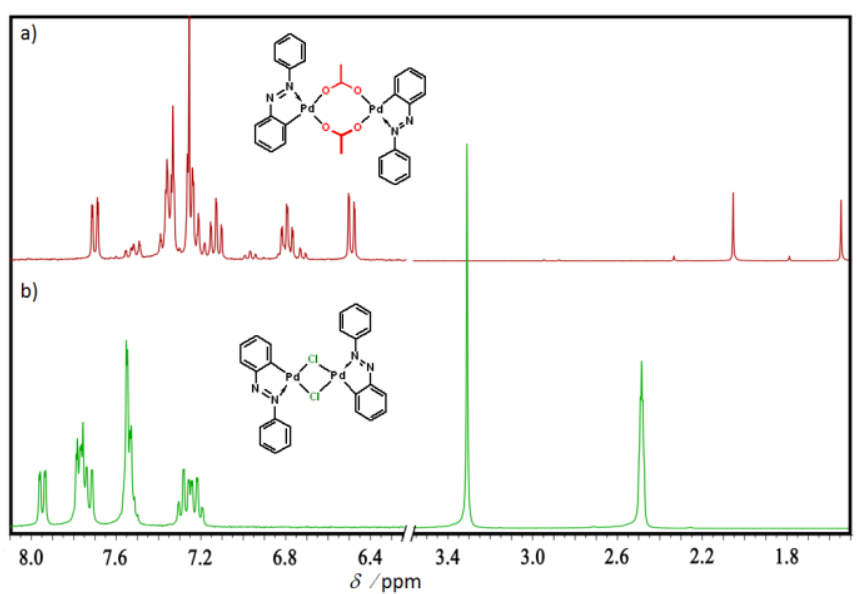

Figure S33. ${ }^{1} \mathrm{H}-\mathrm{NMR}$ spectra of a) precursor $3\left(\mathrm{CDCl}_{3}\right)$ and $b)$ product $5\left(\mathrm{DMSO}_{-} \mathrm{d}_{6}\right)$ obtained from 3 by anion substitution using $\mathrm{LiCl}$.

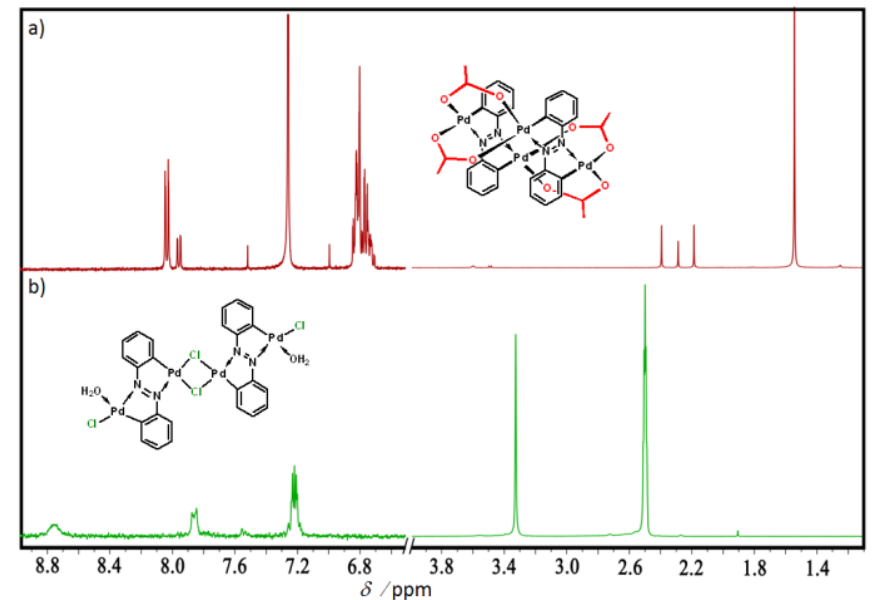

Figure S35. ${ }^{1} \mathrm{H}-\mathrm{NMR}$ spectra of a) precursor 11 $\left(\mathrm{CDCl}_{3}\right)$ and $\left.\mathrm{b}\right)$ product 9 (DMSO-d $)$ obtained from 11 by anion substitution using $\mathrm{LiCl}$.

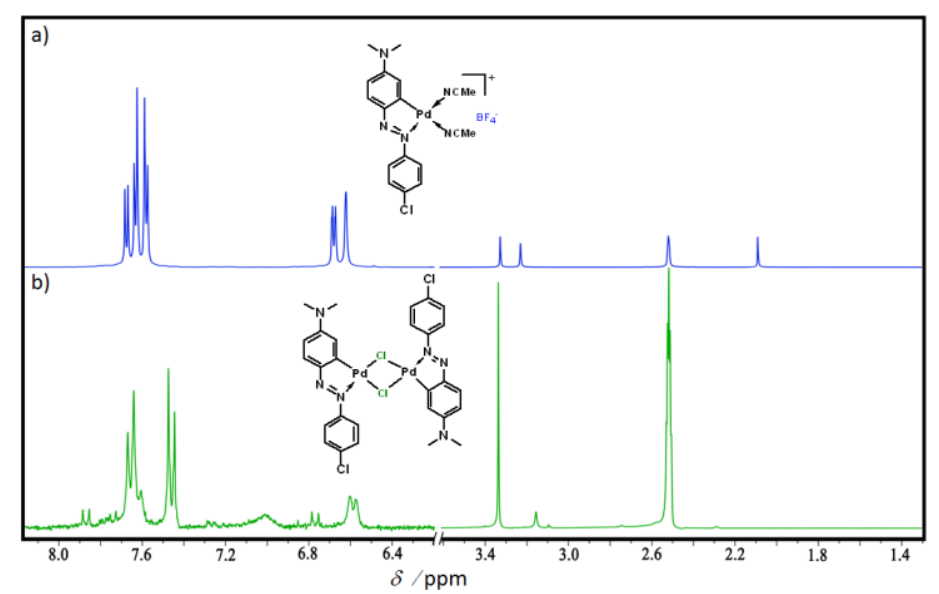

Figure S32. ${ }^{1} \mathrm{H}-\mathrm{NMR}$ (DMSO-d 6 ) spectra of a) precursor 2 and b) product $\mathbf{6}$ obtained from $\mathbf{2}$ by anion substitution using $\mathrm{LiCl}$.

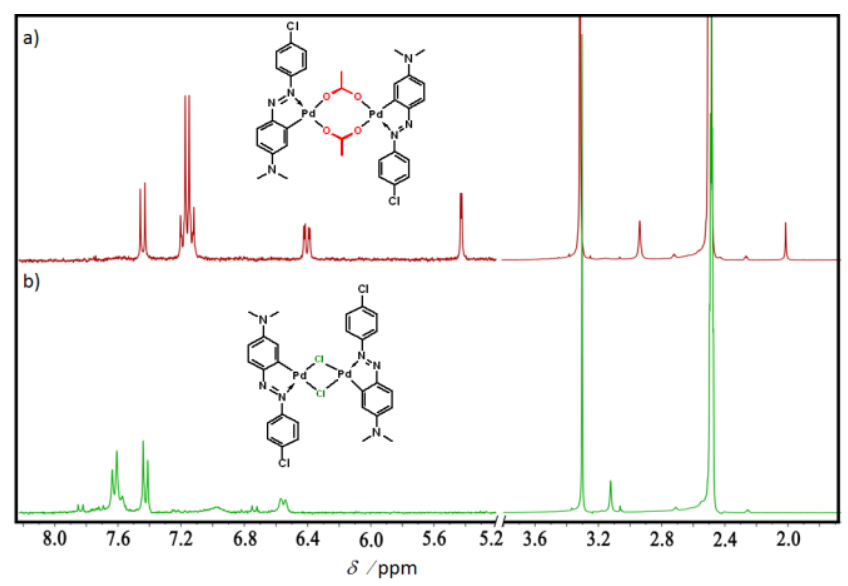

Figure S34. ${ }^{1} \mathrm{H}-\mathrm{NMR}$ (DMSO-d6) spectra of: a) precursor 4 and b) product $\mathbf{6}$ obtained from $\mathbf{4}$ by anion substitution using $\mathrm{LiCl}$.

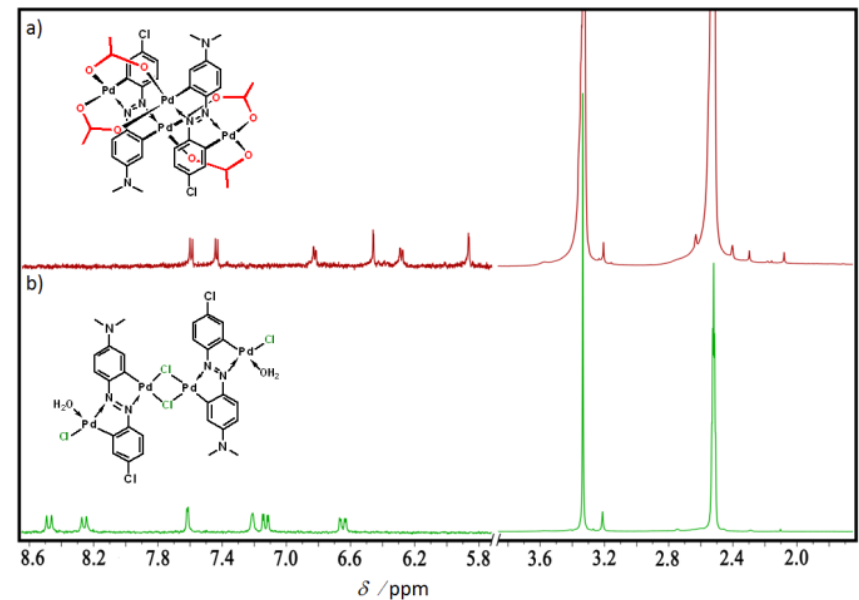

Figure S36. ${ }^{1} \mathrm{H}-\mathrm{NMR}\left(\mathrm{DMSO}-\mathrm{d}_{6}\right.$ ) spectra of a) precursor 12 and b) product $\mathbf{1 0}$ obtained from $\mathbf{1 2}$ by anion substitution using $\mathrm{LiCl}$. 


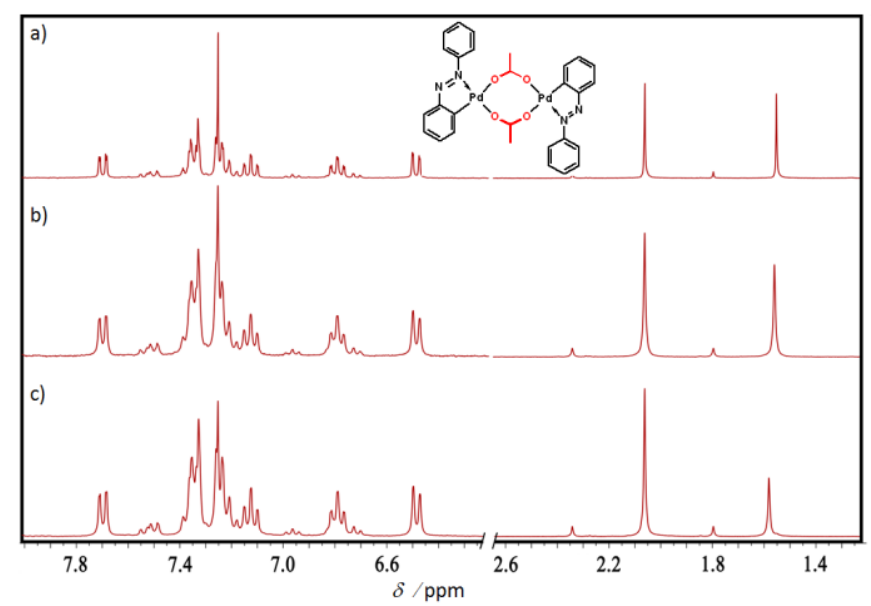

Figure S37. ${ }^{1} \mathrm{H}-\mathrm{NMR}\left(\mathrm{CDCl}_{3}\right)$ spectra of product $\mathbf{3}$ obtained by anion substitution using $\mathrm{NaOAc}$ from: a) $\mathbf{1}$, b) 5 and c) 7 .

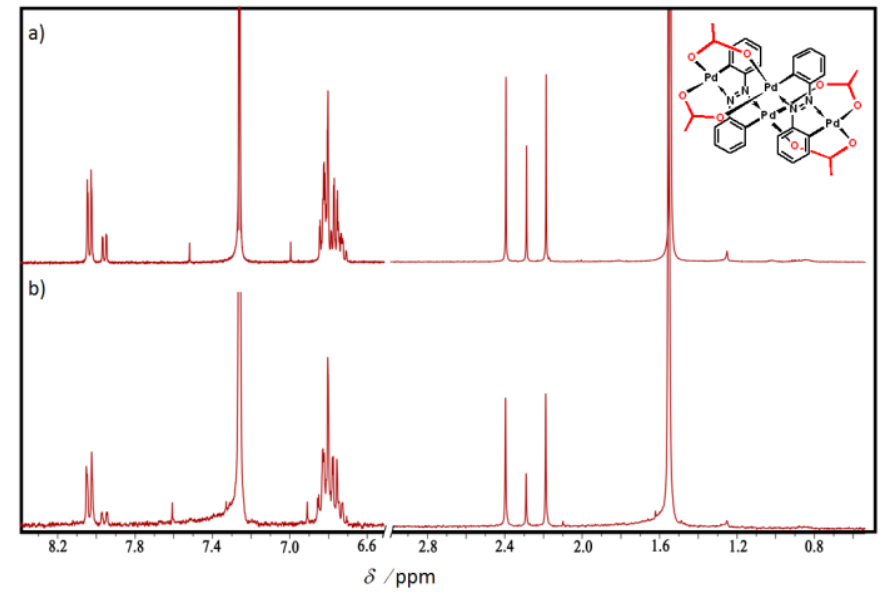

Figure S39. ${ }^{1} \mathrm{H}-\mathrm{NMR}\left(\mathrm{CDCl}_{3}\right)$ spectra of product $\mathbf{1 1}$ obtained by anion substitution using $\mathrm{NaOAc}$ from: a) 9 and b) $\mathbf{1 3}$.

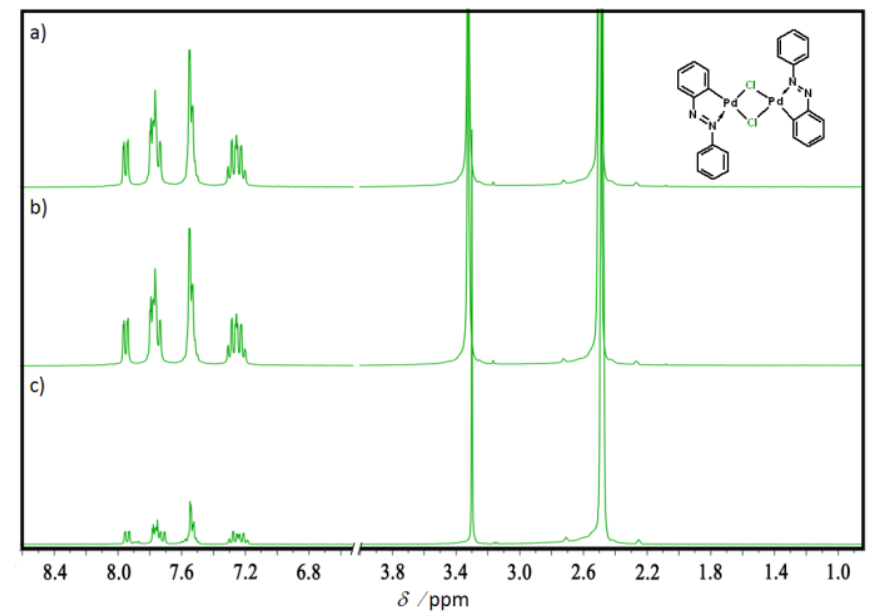

Figure S41. ${ }^{1} \mathrm{H}-\mathrm{NMR}$ (DMSO-d 6 ) spectra of product 5 obtained by anion substitution using $\mathrm{LiCl}$ from: a) 1, b) 3 and c) 7.

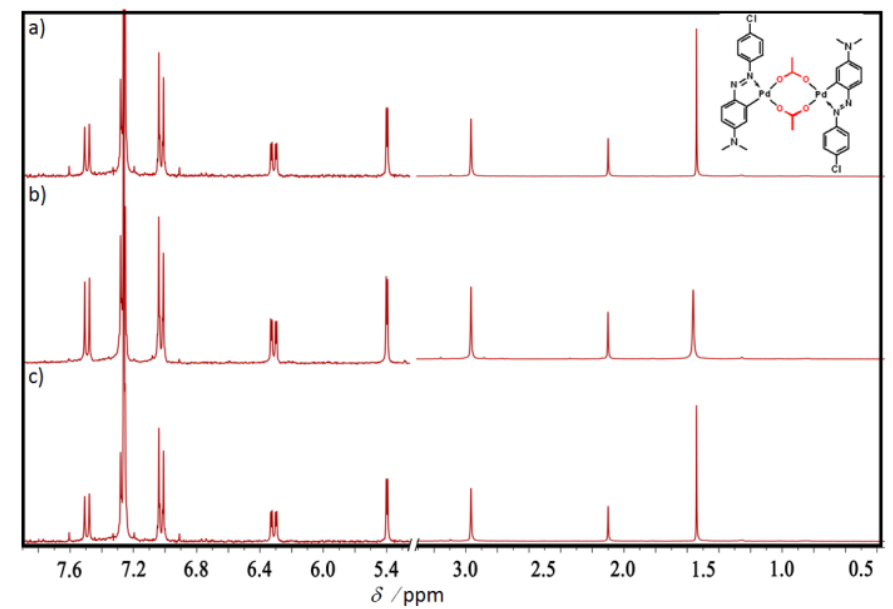

Figure S38. ${ }^{1} \mathrm{H}-\mathrm{NMR}\left(\mathrm{CDCl}_{3}\right)$ spectra of product 4 obtained by anion substitution using $\mathrm{NaOAc}$ from: a) 2, b) 6 and c) 8 .

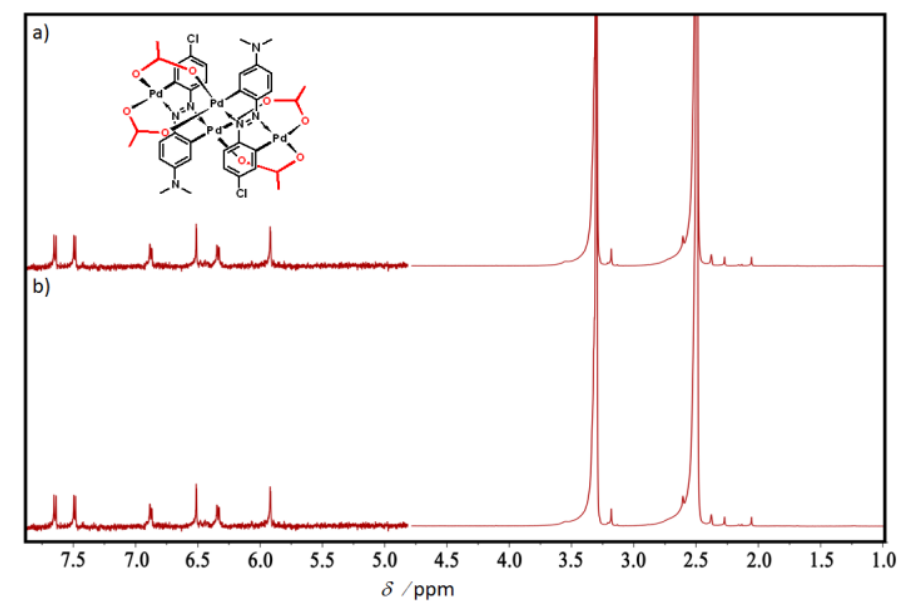

Figure S40. ${ }^{1} \mathrm{H}-\mathrm{NMR}$ (DMSO- $\mathrm{d}_{6}$ ) spectra of product $\mathbf{1 2}$ obtained by anion substitution using $\mathrm{NaOAc}$ from: a) $\mathbf{1 0}$ and b) 14.

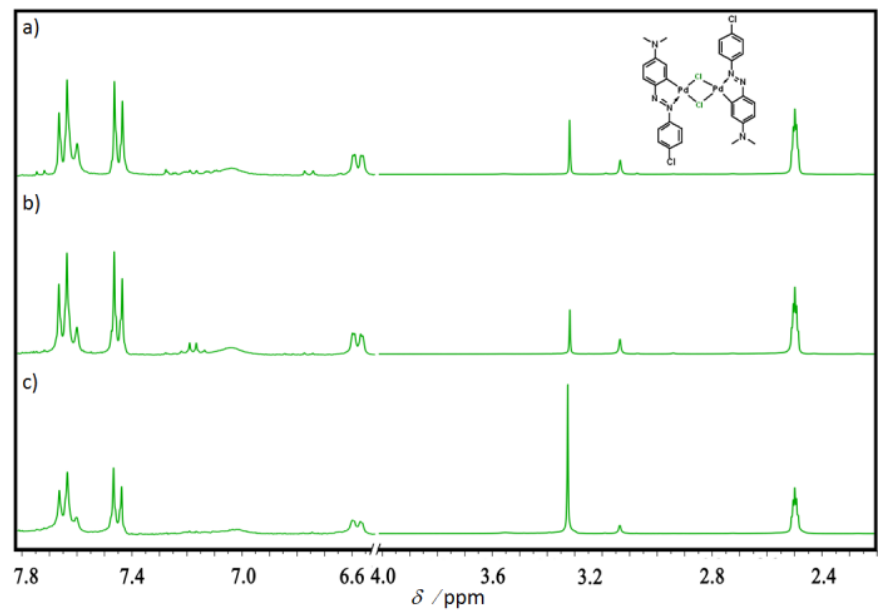

Figure S42. ${ }^{1} \mathrm{H}-\mathrm{NMR}$ (DMSO-d6) spectra of product 6 obtained by anion substitution using $\mathrm{LiCl}$ from: a) 2, b) 4 and c) 8 . 

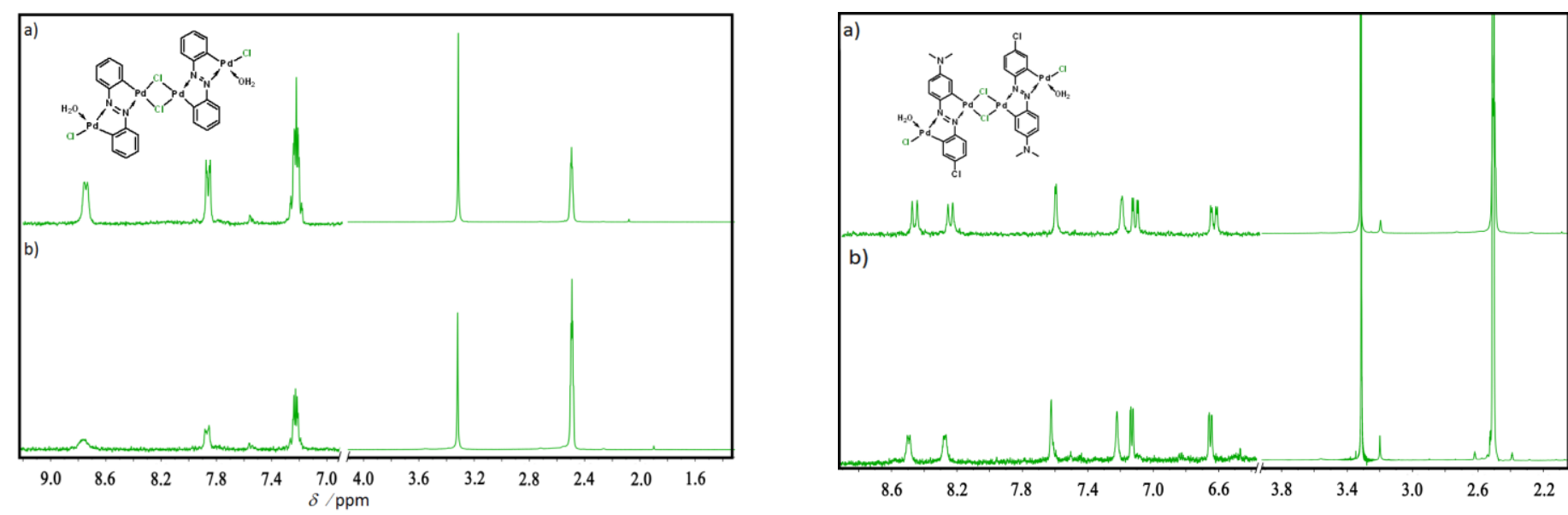

Figure S43. ${ }^{1} \mathrm{H}-\mathrm{NMR}\left(\mathrm{DMSO}_{-} \mathrm{d}_{6}\right)$ spectra of product 9 Figure $\mathbf{S 4 4} .{ }^{1} \mathrm{H}-\mathrm{NMR}$ (DMSO-d6) spectra of product 10 obtained by anion substitution using LiCl from: a) $\mathbf{1 1}$ obtained by anion substitution using LiCl from: a) $\mathbf{1 2}$ and b) 14 . and b) 13 . 


\section{IR SPECTROSCOPY}

Table S4. IR vibrations of select groups of acetate and acetylacetonate complexes

\begin{tabular}{|c|c|c|c|c|c|}
\hline & $v_{a s}(\mathbf{C O}) / \mathbf{c m}^{-\mathbf{1}}$ & $\boldsymbol{v}_{\text {sym }}(\mathbf{C O}) / \mathbf{c m}^{-\mathbf{1}}$ & & $\boldsymbol{v}_{\text {as }}(\mathbf{C O}) / \mathbf{c m}^{-\mathbf{1}}$ & $\boldsymbol{v}_{\text {sym }}(\mathbf{C O}) / \mathbf{c m}^{\mathbf{- 1}}$ \\
\hline NaOAc & 1568 & 1417 & $\mathbf{L i}(\mathbf{a c a c})$ & 1587 & 1516 \\
\hline $\mathbf{3}$ & 1556 & 1412 & $\mathbf{7}$ & 1569 & 1511 \\
\hline $\mathbf{4}$ & 1564 & 1411 & $\mathbf{8}$ & 1579 & 1513 \\
\hline $\mathbf{1 1}$ & 1555 & 1414 & $\mathbf{1 3}$ & 1567 & 1520 \\
\hline $\mathbf{1 2}$ & 1562 & 1415 & $\mathbf{1 4}$ & 1573 & 1512 \\
\hline
\end{tabular}

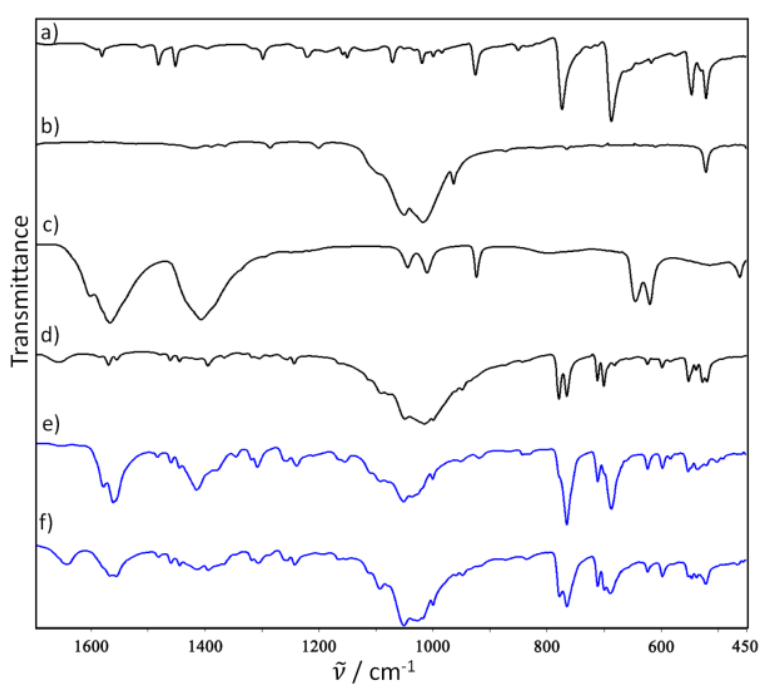

Figure S45. FTIR spectra of: a) L1, $\left[\mathrm{Pd}(\mathrm{MeCN})_{4}\right]\left[\mathrm{BF}_{4}\right]_{2}$, c) $\mathrm{NaOAc}$, d) crude product 1 product 9 synthesised by ILAG using $\mathrm{NaOAc}$ and $\mathrm{H}_{2} \mathrm{O}$ as

synthesised by IAG using $\mathrm{NaOAc}_{\text {and }} \mathrm{NaBF}_{4}$ as additives, additives and d) pure product 9 after washing with water.

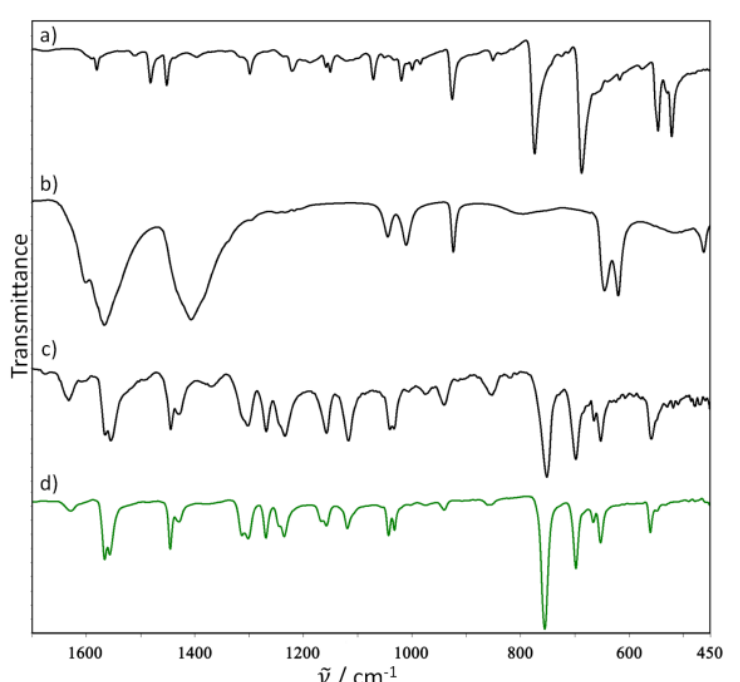

e) pure product $\mathbf{1}$ after washing with water and f) recrystallized from $\mathrm{MeCN}$. 


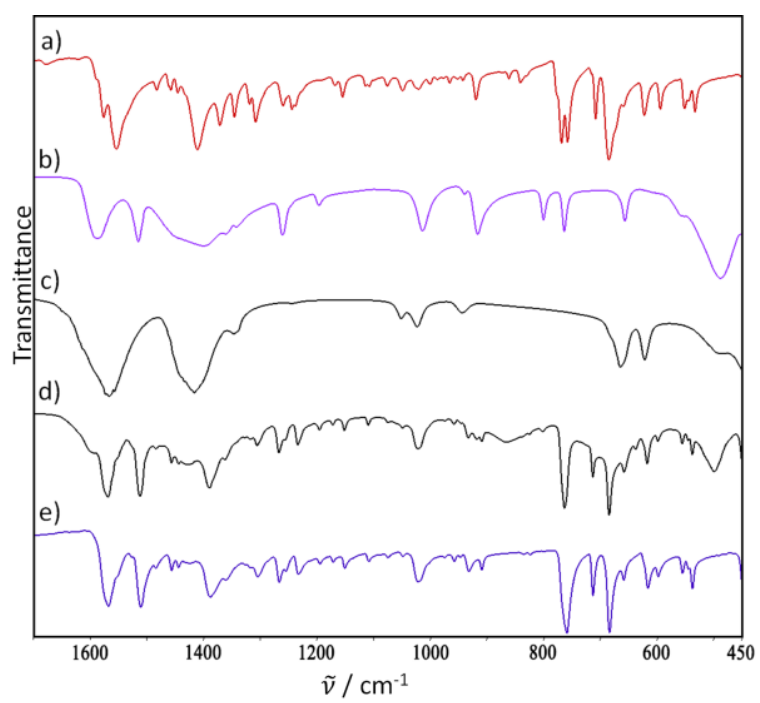

Figure S47. FTIR spectra of: a) precursor 3, b) Li(acac), Figure S48. FTIR spectra of: a) precursor 4, b) Li(acac), c) c) byproduct $\mathrm{LiOAc}, \mathrm{d}$ ) resulting mixture of product $\mathbf{7}$, byproduct $\mathrm{LiOAc}, \mathrm{d}$ ) resulting mixture of product $8, \mathrm{Li}(\mathrm{acac})$,

$\mathrm{Li}(\mathrm{acac}), \mathrm{LiOAc}$ and e) pure product 7 after washing with LiOAc and e) pure product $\mathbf{8}$ after washing with water. water.

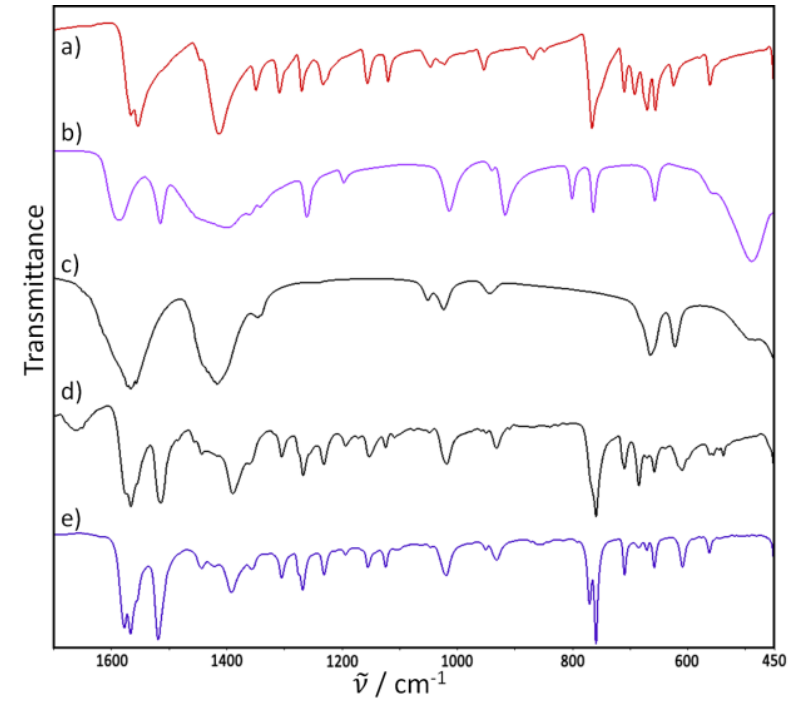

Figure S49. FTIR spectra of: a) precursor 11, b) Li(acac), c) byproduct LiOAc, d) resulting mixture of product 13, $\mathrm{Li}(\mathrm{acac}), \mathrm{LiOAc}$ and e) pure product $\mathbf{1 3}$ after washing with water.
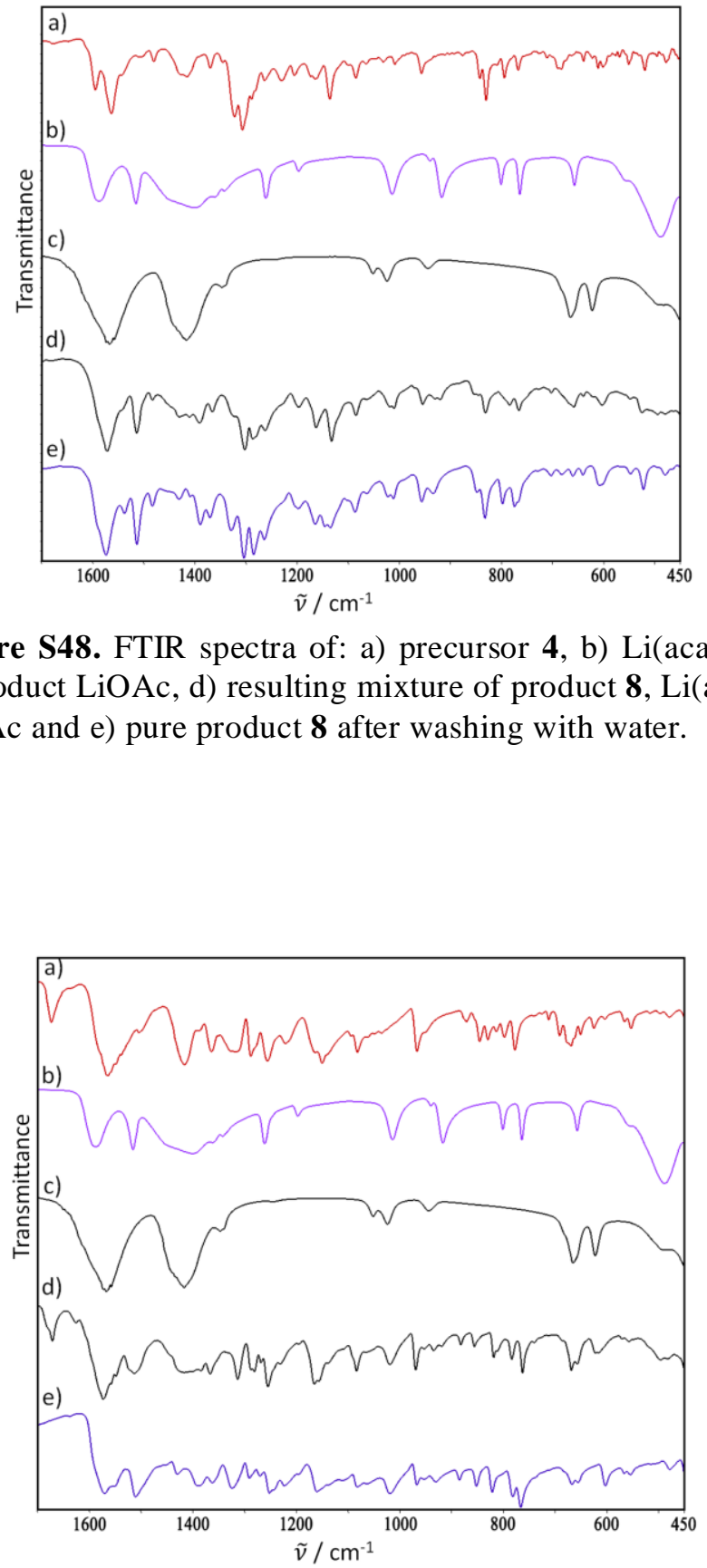

Figure S50. FTIR spectra of: a) precursor 12, b) Li(acac), c) byproduct LiOAc, d) resulting mixture of product 14, $\mathrm{Li}(\mathrm{acac}), \mathrm{LiOAc}$ and e) pure product 14 after washing with water. 


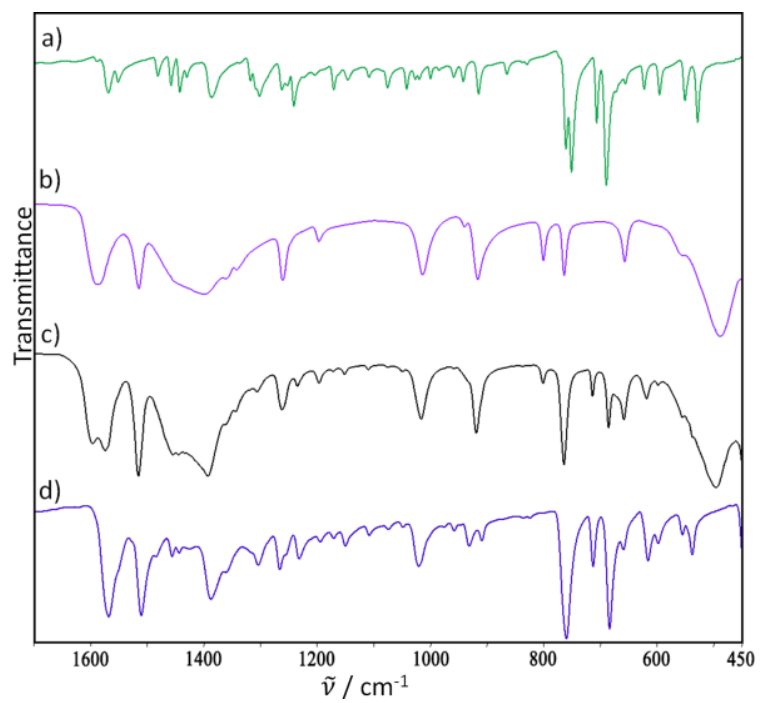

Figure S51. FTIR spectra of: a) precursor 5, b) $\operatorname{Li}(a c a c)$ c) resulting mixture of product $7, \mathrm{Li}(\mathrm{acac}), \mathrm{LiCl}$ and $\mathrm{d}$ ) pure product 7 after washing with water.

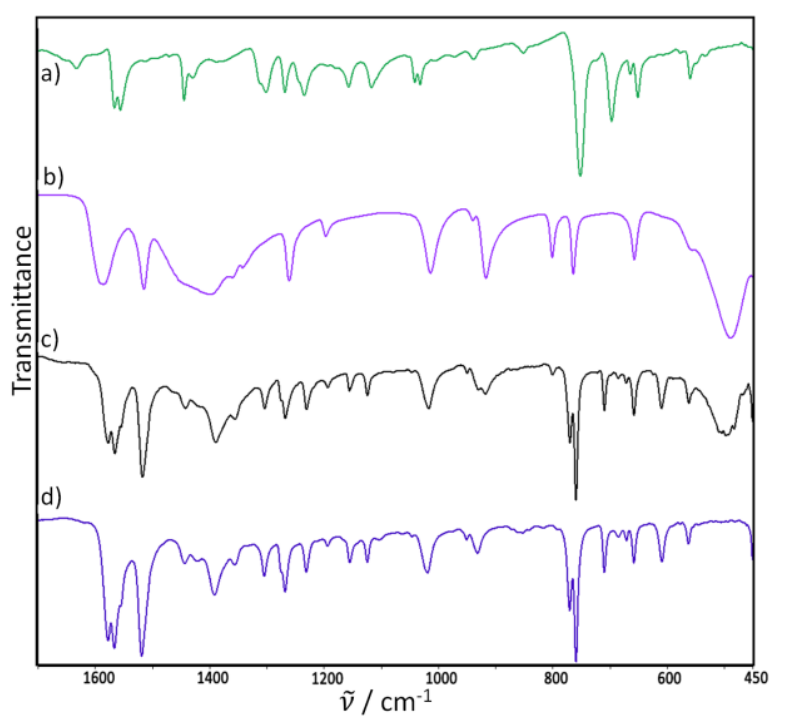

Figure S53. FTIR spectra of: a) precursor 9, b) Li(acac), c) resulting mixture of product $13, \mathrm{Li}(\mathrm{acac}), \mathrm{LiCl}$ and $\mathrm{d}$ ) pure product $\mathbf{1 3}$ after washing with water.

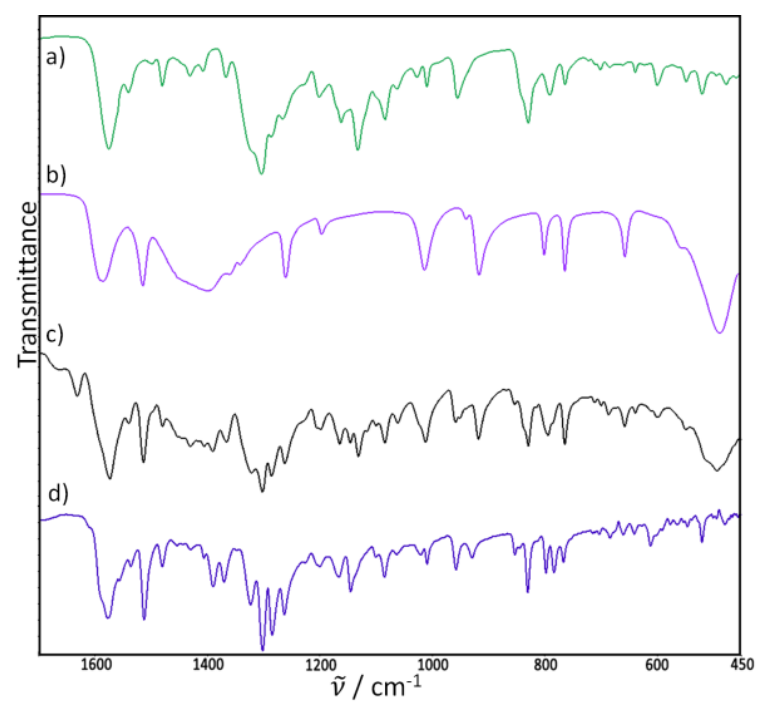

Figure S52. FTIR spectra of: a) precursor 6, b) Li(acac), c) resulting mixture of product $\mathbf{8}, \mathrm{Li}(\mathrm{acac}), \mathrm{LiCl}$ and d) pure product $\mathbf{8}$ after washing with water.

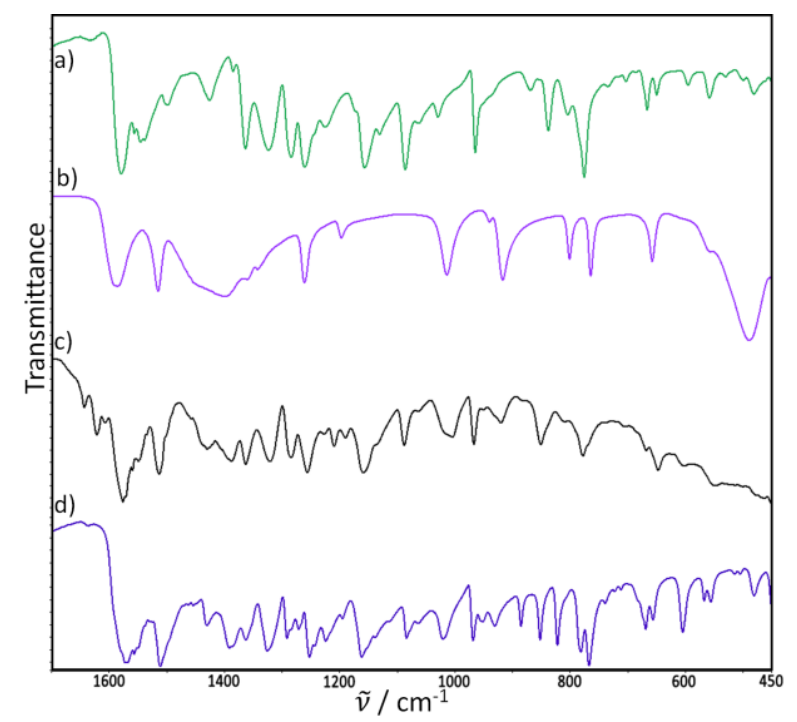

Figure S54. FTIR spectra of: a) precursor 10, b) Li(acac), c) resulting mixture of product $14, \mathrm{Li}(\mathrm{acac}), \mathrm{LiCl}$ and d) pure product $\mathbf{1 4}$ after washing with water. 


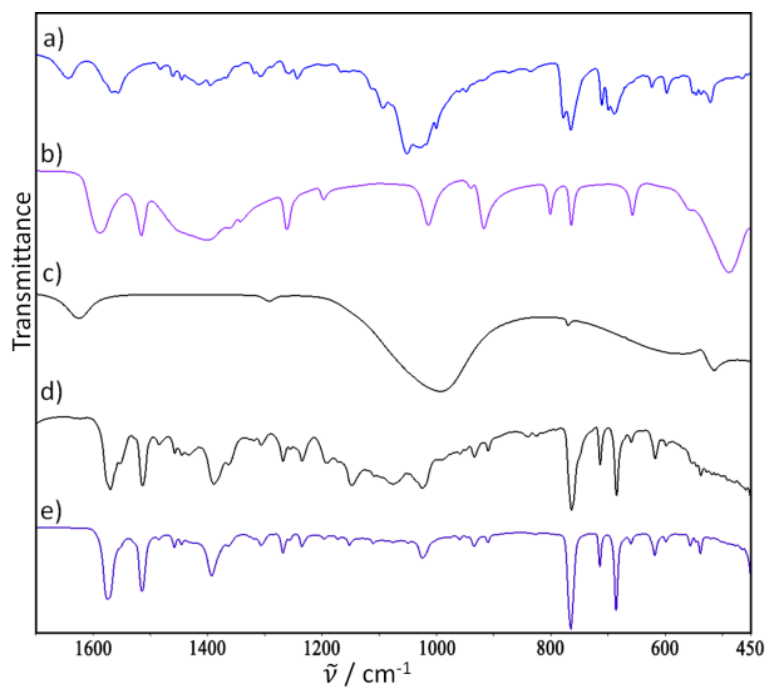

Figure S55. FTIR spectra of: a) precursor 1, b) Li(acac), Figure S56. FTIR spectra of: a) 2, b) Li(acac), c) byproduct c) byproduct $\mathrm{LiBF}_{4}$, d) resulting mixture of $7, \mathrm{Li}(\mathrm{acac}), \mathrm{LiBF}_{4}$, d) resulting mixture of product $\mathbf{8}, \mathrm{Li}(\mathrm{acac}), \mathrm{LiBF}_{4}$ and $\mathrm{LiBF}_{4}$ and e) pure product 7 after washing with water.

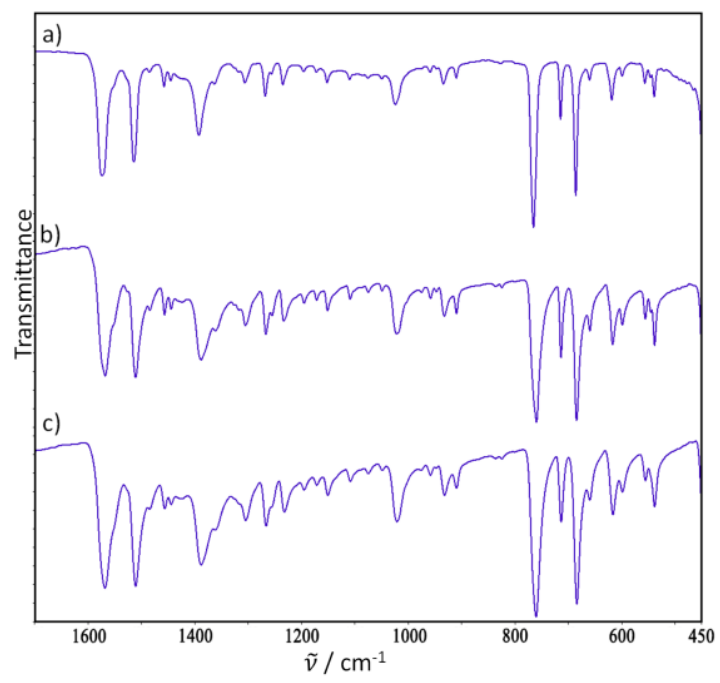

Figure S57. FTIR spectra of purified product 7 obtained by ion exchange reaction with $\mathrm{Li}(\mathrm{acac})$ from: a) 1, b) 3 and c) 5 .

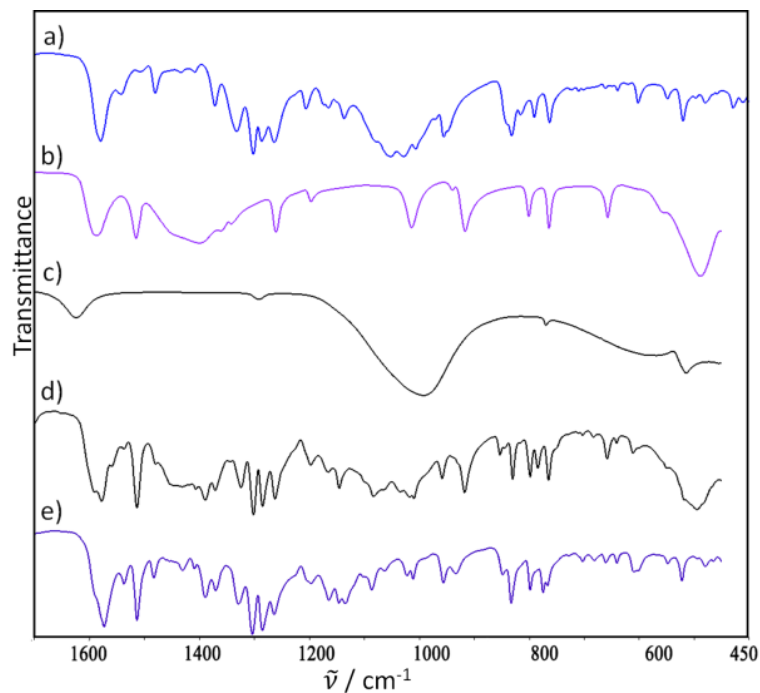

e) pure product $\mathbf{8}$ after washing with water.

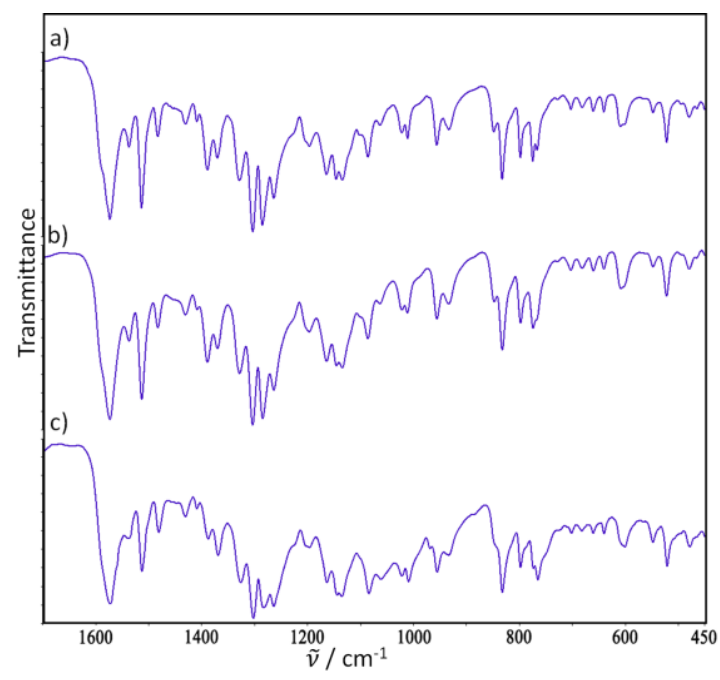

Figure S58. FTIR spectra of purified product 8 obtained by ion exchange reaction with $\operatorname{Li}(\mathrm{acac})$ from: a) $\mathbf{2}$, b) $\mathbf{4}$ and c) $\mathbf{6}$. 


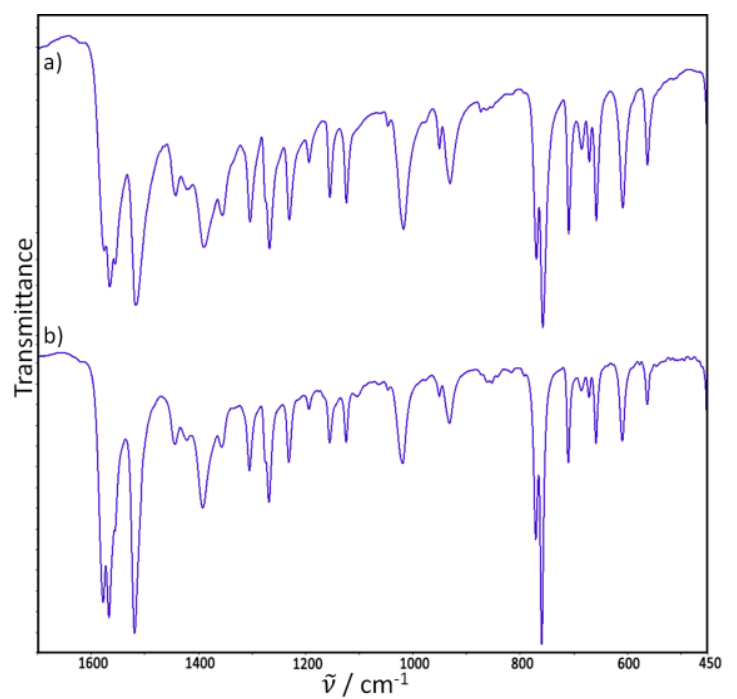

Figure S59. FTIR spectra of purified product 13 obtained by ion exchange reaction with $\operatorname{Li}(\mathrm{acac})$ from: a) $\mathbf{9}$ and b) 11.

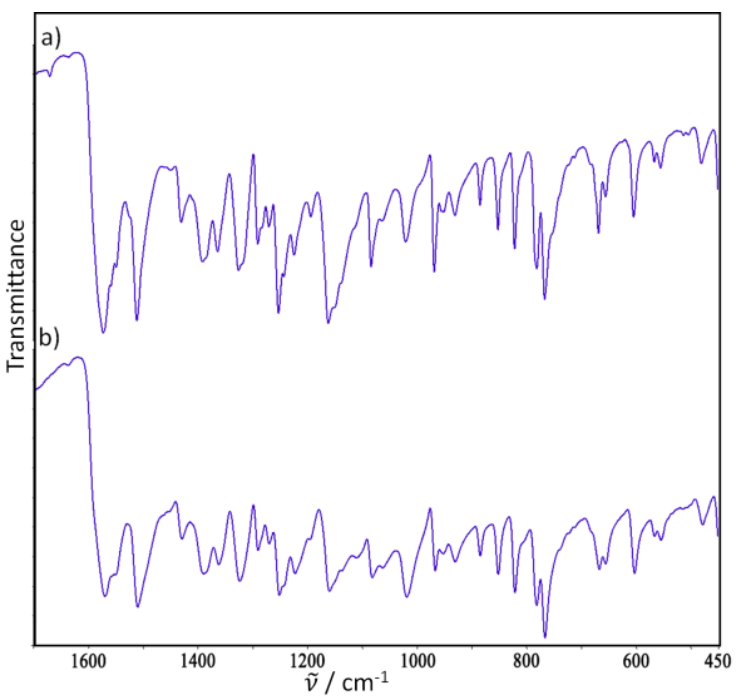

Figure S60. FTIR spectra of purified product 14 obtained by ion exchange reaction with $\mathrm{Li}(\mathrm{acac})$ from: a) $\mathbf{1 0}$ and b) $\mathbf{1 2}$.

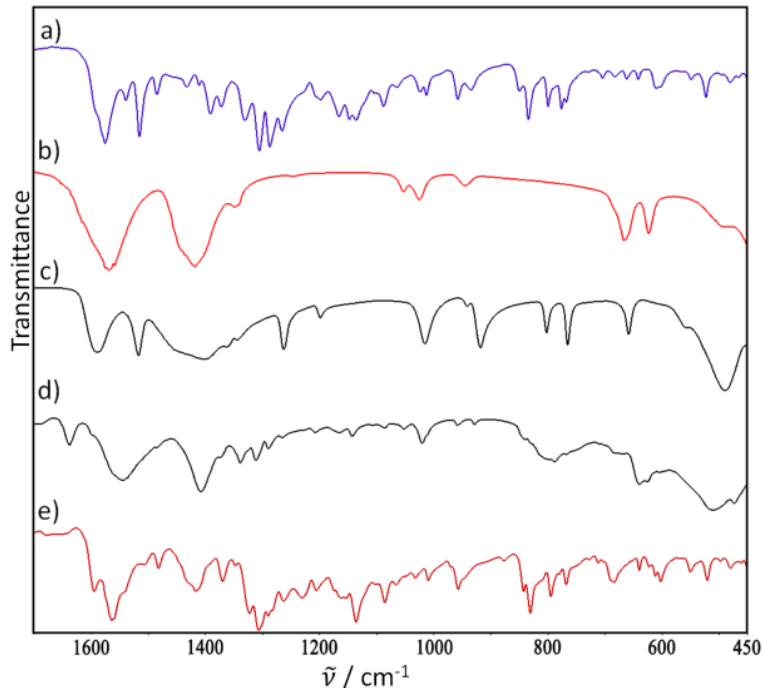

Figure S61. FTIR spectra of: a) precursor 7, b) NaOAc, c) Figure S62. FTIR spectra of: a) precursor 8, b) $\mathrm{NaOAc}$, c) byproduct $\mathrm{Na}(\mathrm{acac})$, d) resulting mixture of product 3, byproduct $\mathrm{Na}(\mathrm{acac})$, d) resulting mixture of product $\mathbf{4}$, $\mathrm{NaOAc}, \mathrm{Na}(\mathrm{acac})$ and e) pure product 3 after washing with water.

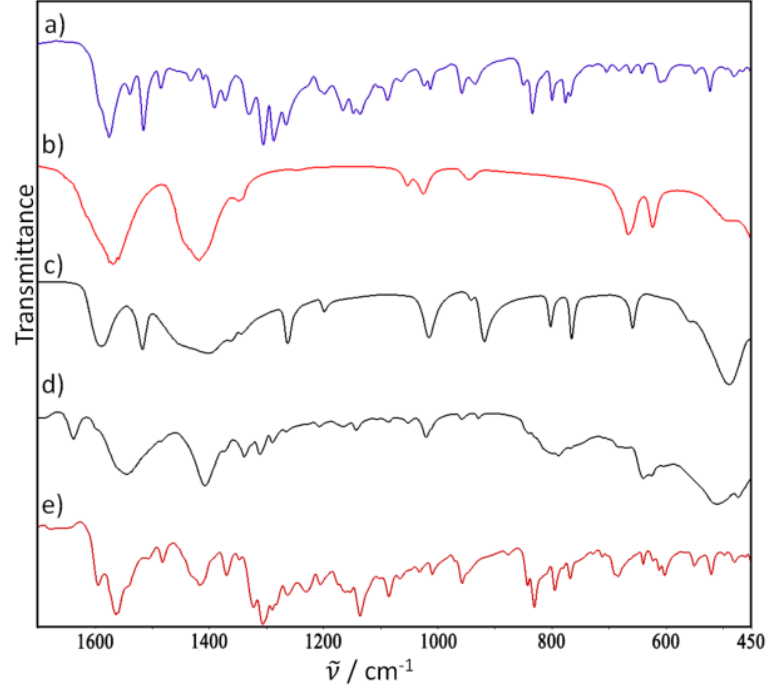

$\mathrm{NaOAc}, \mathrm{Na}(\mathrm{acac})$ and e) pure product 4 after washing with water. 


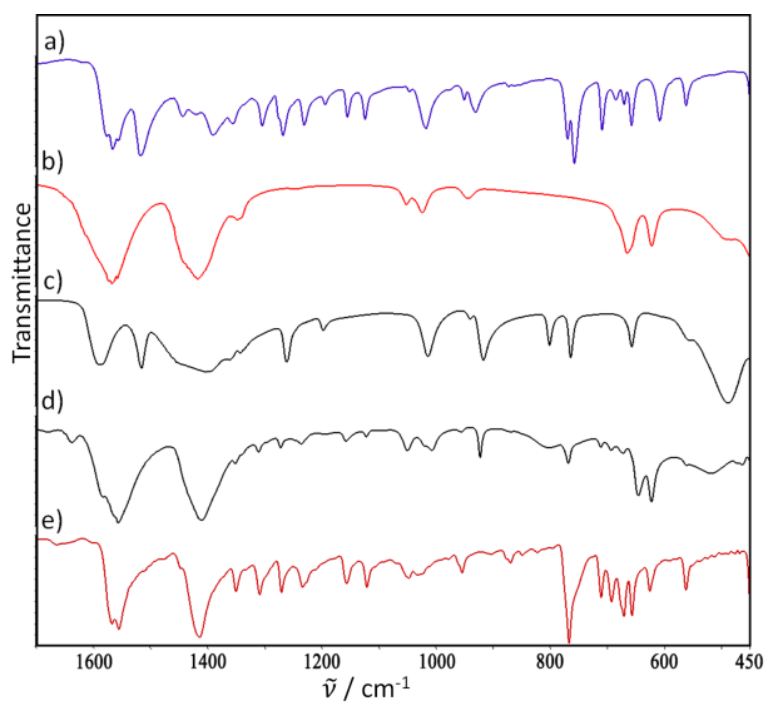

Figure S63. FTIR spectra of: a) precursor 13, b) NaOAc, Figure S64. FTIR spectra of: a) precursor 14, b) NaOAc, c) c) byproduct $\mathrm{Na}(\mathrm{acac})$, d) resulting mixture of product 11, byproduct $\mathrm{Na}(\mathrm{acac})$, d) resulting mixture of 12, $\mathrm{NaOAc}$,

$\mathrm{NaOAc}, \mathrm{Na}(\mathrm{acac})$ and e) pure product 11 after washing $\mathrm{Na}(\mathrm{acac})$ and e) pure product $\mathbf{1 2}$ after washing with water. with water.

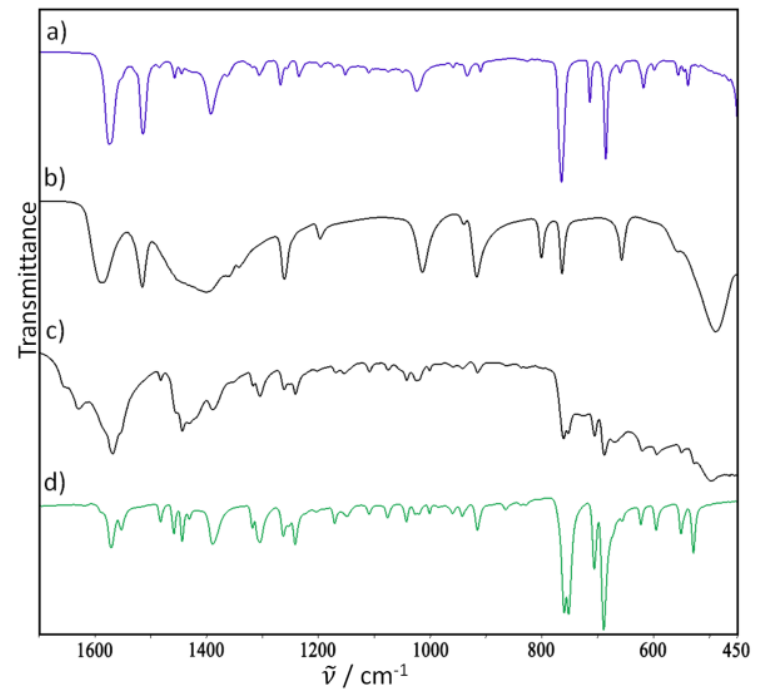

Figure S65. FTIR spectra of: a) precursor 7, b) byproduct $\mathrm{Li}(\mathrm{acac}), \mathrm{c})$ resulting mixture of product $\mathbf{5}, \mathrm{LiCl}, \mathrm{Li}(\mathrm{acac})$ and d) pure 5 after washing with water.
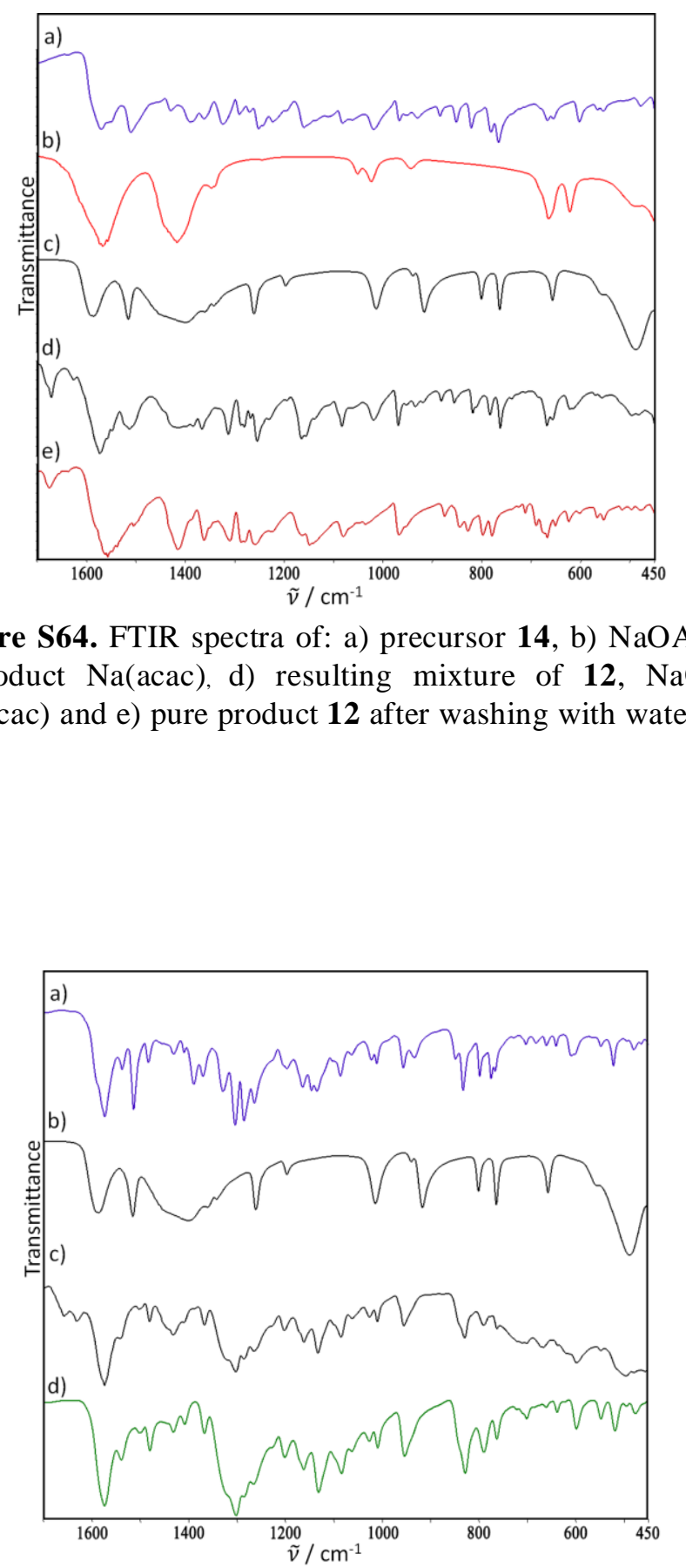

Figure S66. FTIR spectra of: a) precursor 8 , b) byproduct $\mathrm{Li}(\mathrm{acac}), \mathrm{c})$ resulting mixture of product $\mathbf{6}, \mathrm{LiCl}, \mathrm{Li}(\mathrm{acac})$ and d) pure $\mathbf{6}$ after washing with water. 


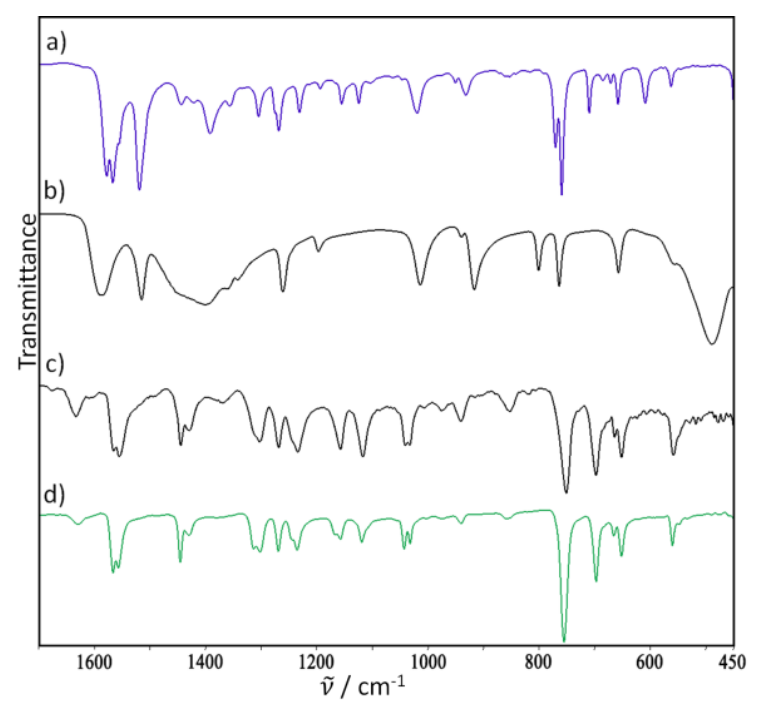

Figure S67. FTIR spectra of: a) 13, b) byproduct Li(acac), c) resulting mixture of product $\mathbf{9}, \mathrm{LiCl}, \mathrm{Li}(\mathrm{acac})$ and $\mathrm{d}$ ) pure product 9 after washing with water.

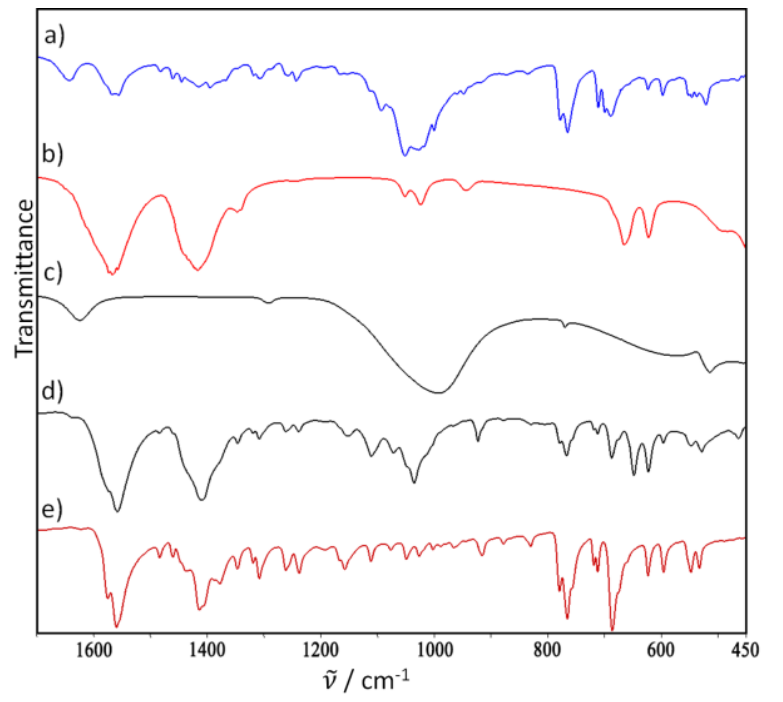

Figure S69. FTIR spectra of: a) precursor 1, b) NaOAc, c) Figure S70. FTIR spectra of: a) precursor 2, b) NaOAc, c) byproduct $\mathrm{NaBF}_{4}$, d) resulting mixture of product $\mathbf{3}$, byproduct $\mathrm{NaBF}_{4}$, d) resulting mixture of product $4, \mathrm{NaOAc}$,

$\mathrm{NaOAc}, \mathrm{NaBF}_{4}$ and e) pure product 3 after washing with $\mathrm{NaBF}_{4}$ and e) pure product $\mathbf{4}$ after washing with water. water.

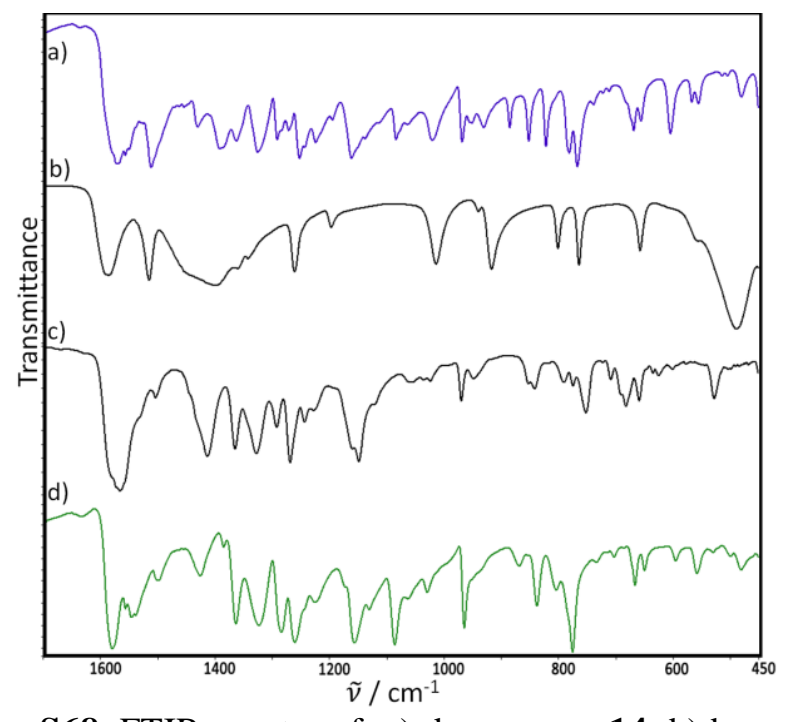

Figure S68. FTIR spectra of: a) d precursor 14, b) byproduct $\mathrm{Li}(\mathrm{acac}), \mathrm{c})$ resulting mixture of product $\mathbf{1 0}, \mathrm{LiCl}, \mathrm{Li}(\mathrm{acac})$ and d) pure product $\mathbf{1 0}$ after washing with water.

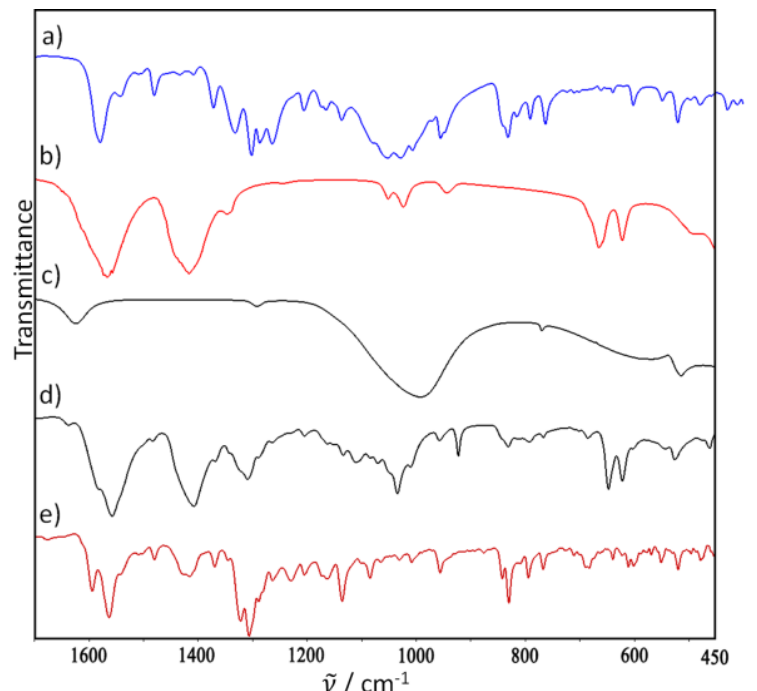




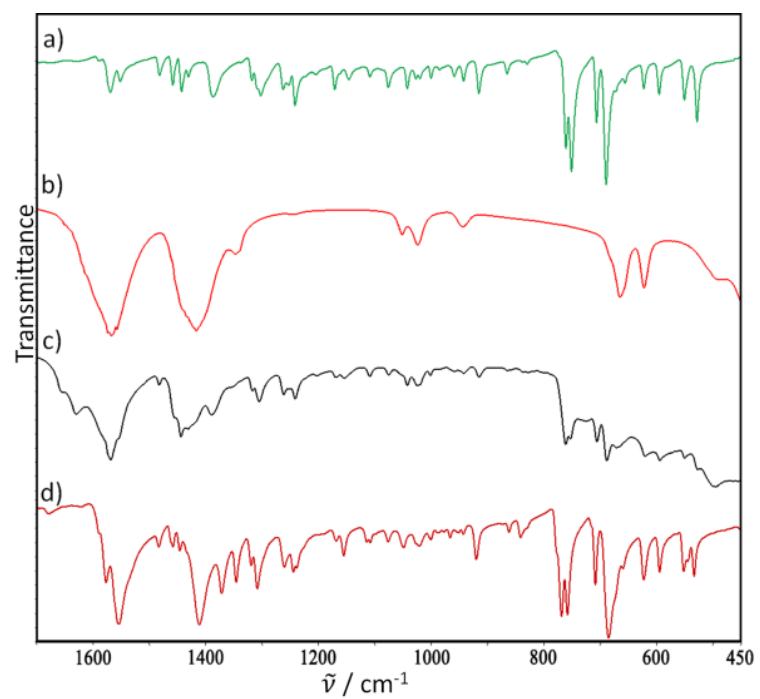

Figure S71. FTIR spectra of: a) precursor 5, b) $\mathrm{NaOAc}$, c) resulting mixture of $\mathbf{3}, \mathrm{NaOAc}$, byproduct $\mathrm{NaCl}$ and d) pure product 3 after washing with water.

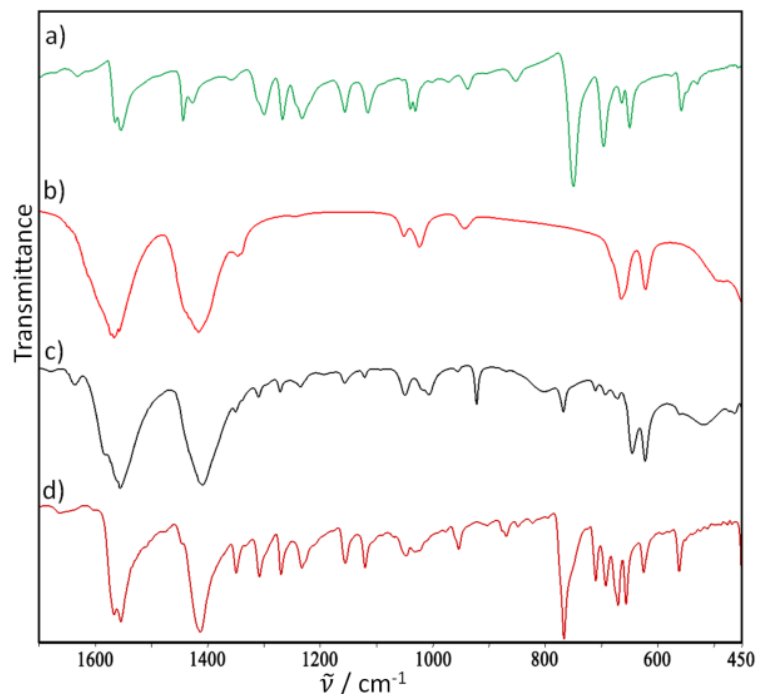

Figure S73. FTIR spectra of: a) precursor 9, b) $\mathrm{NaOAc}$, c) resulting mixture of product $11, \mathrm{NaOAc}$, byproduct $\mathrm{NaCl}$ and d) pure product $\mathbf{1 1}$ after washing with water.

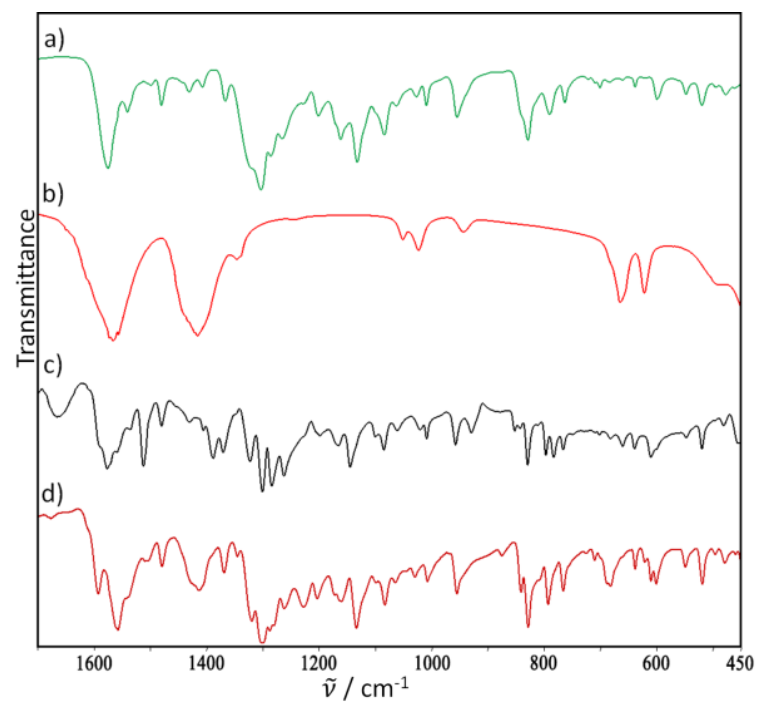

Figure S72. FTIR spectra of: a) precursor 6, b) $\mathrm{NaOAc}$, c) resulting mixture of product $4, \mathrm{NaOAc}$, byproduct $\mathrm{NaCl}$ and d) pure product $\mathbf{4}$ after washing with water.

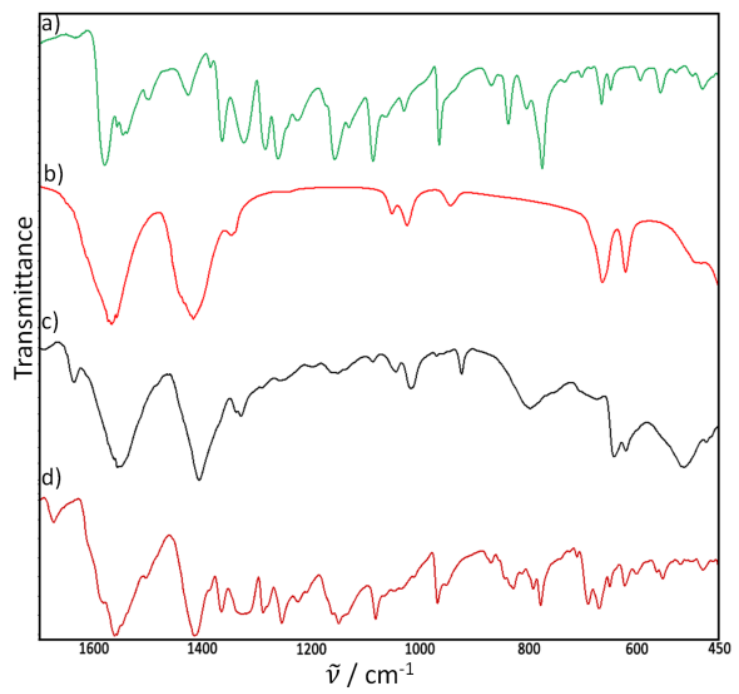

Figure S74. FTIR spectra of: a) precursor 10, b) NaOAc, c) resulting mixture of product $12, \mathrm{NaOAc}$, byproduct $\mathrm{NaCl}$ and d) pure product 12 after washing with water. 


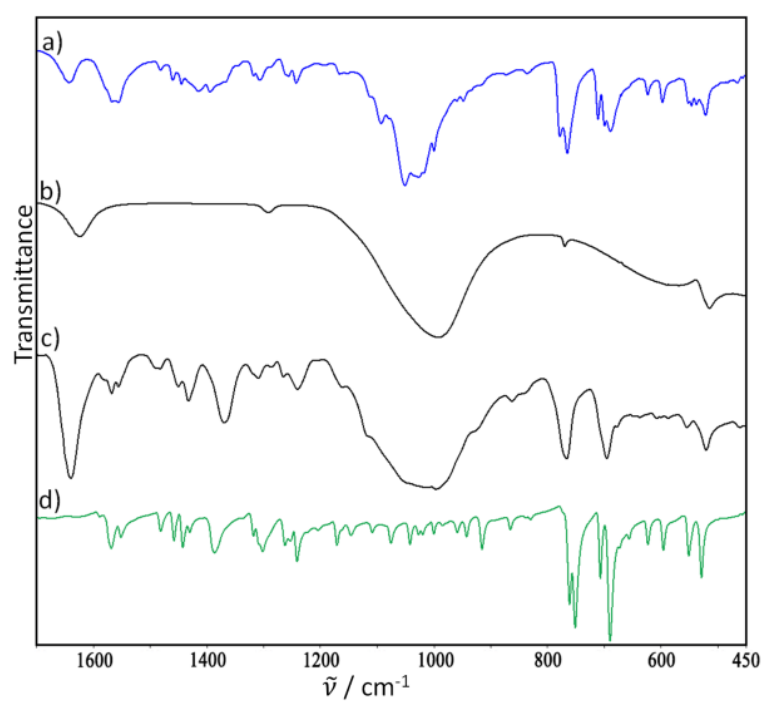

Figure S75. FTIR spectra of: a) precursor 1, b) byproduct $\mathrm{LiBF}_{4}$, c) resulting mixture of product $\mathbf{5}, \mathrm{LiCl}, \mathrm{LiBF}_{4}$ and d) pure product $\mathbf{5}$ after washing with water.

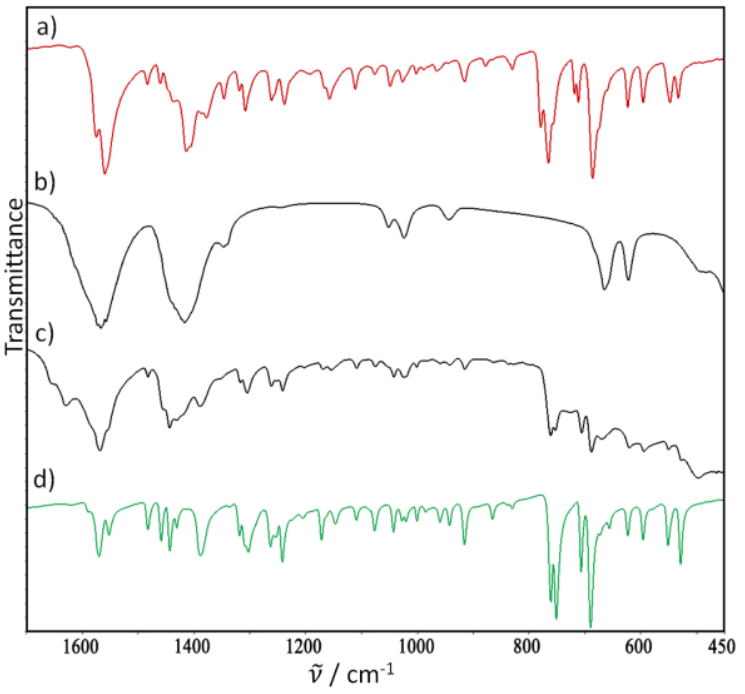

Figure S77. FTIR spectra of: a) precursor 3, b) byproduct $\mathrm{LiOAc}, \mathrm{c})$ resulting mixture of product $\mathbf{5}, \mathrm{LiCl}$, byproduct LiOAc and d) pure product 5 after washing with water.

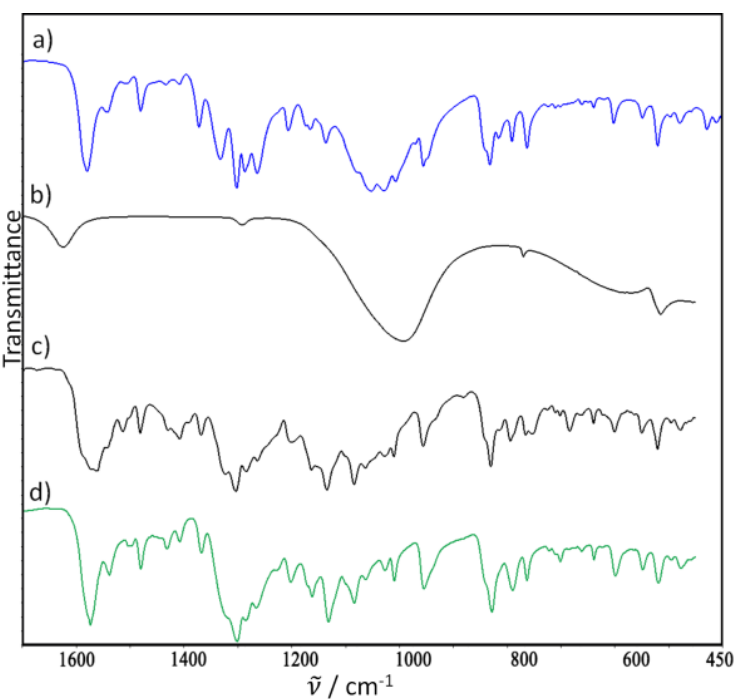

Figure S76. FTIR spectra of: a) precursor 2, b) byproduct $\left.\mathrm{LiBF}_{4}, \mathrm{c}\right)$ resulting mixture of product $\mathbf{6}, \mathrm{LiCl}, \mathrm{LiBF}_{4}$ and d) pure product $\mathbf{6}$ after washing with water.

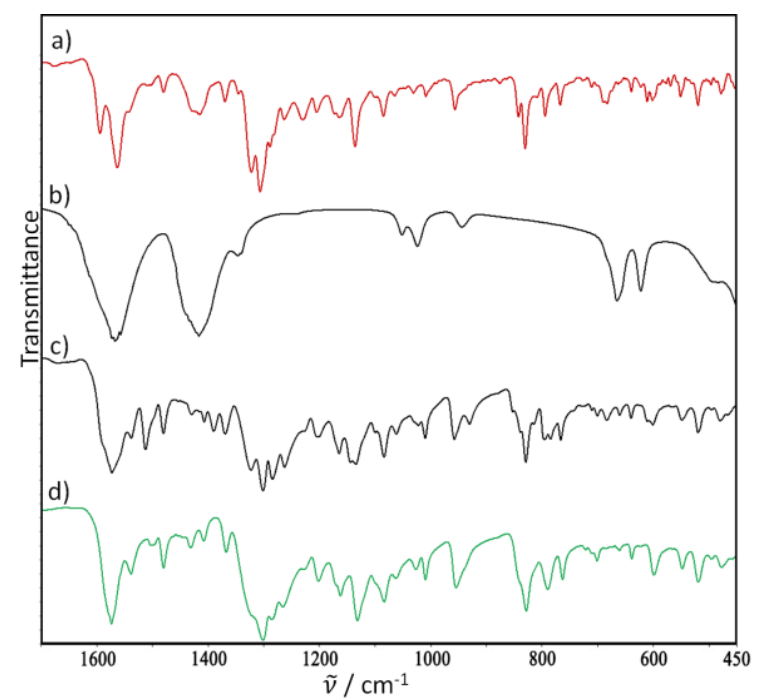

Figure S78. FTIR spectra of: a) precursor 4, b) byproduct $\mathrm{LiOAc}, \mathrm{c})$ resulting mixture of product $\mathbf{6}, \mathrm{LiCl}$, byproduct LiOAc and d) pure product $\mathbf{6}$ after washing with water. 


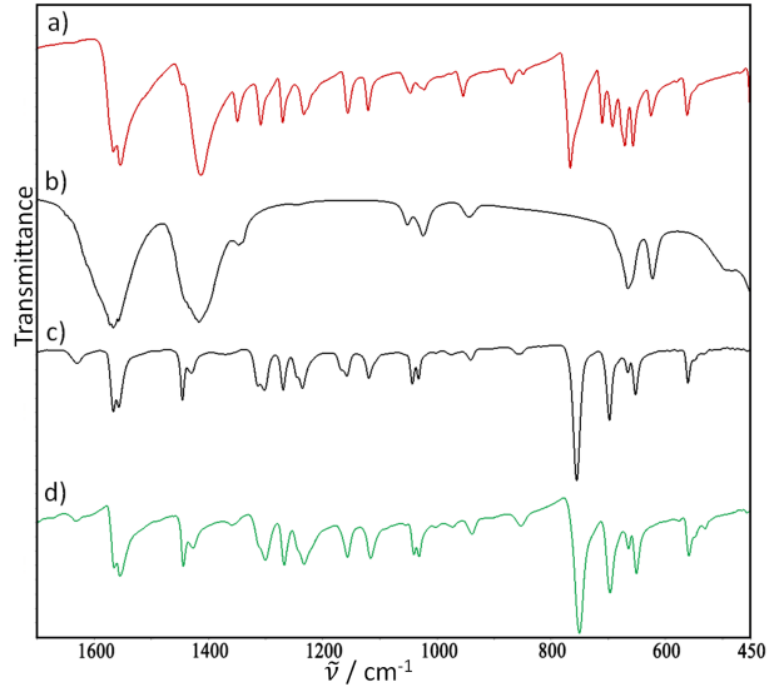

Figure S79. FTIR spectra of: a) precursor 11, b) Figure S80. FTIR spectra of: a) precursor 12, b) byproduct byproduct $\mathrm{LiOAc}, \mathrm{c})$ resulting mixture of product $\mathbf{9}, \mathrm{LiCl}, \mathrm{LiOAc}, \mathrm{c})$ resulting mixture of product $\mathbf{1 0}$, $\mathrm{LiCl}$, byproduct byproduct LiOAc and d) pure product 9 after washing with LiOAc and d) pure product $\mathbf{1 0}$ after washing with water. water.

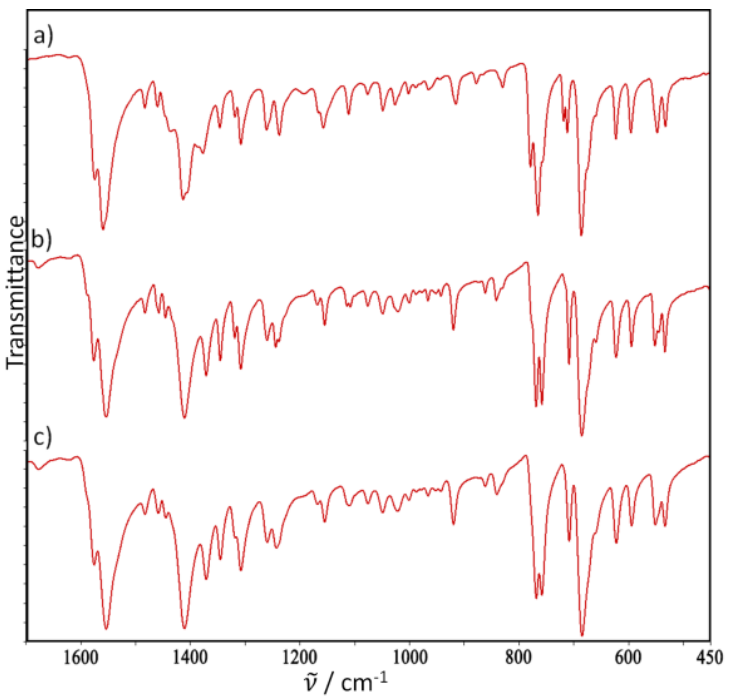

Figure S81. FTIR spectra of purified product 3 obtained by ion exchange reaction with $\mathrm{NaOAc}$ from: a) $\mathbf{1}$, b) $\mathbf{5}$ and c) 7 .
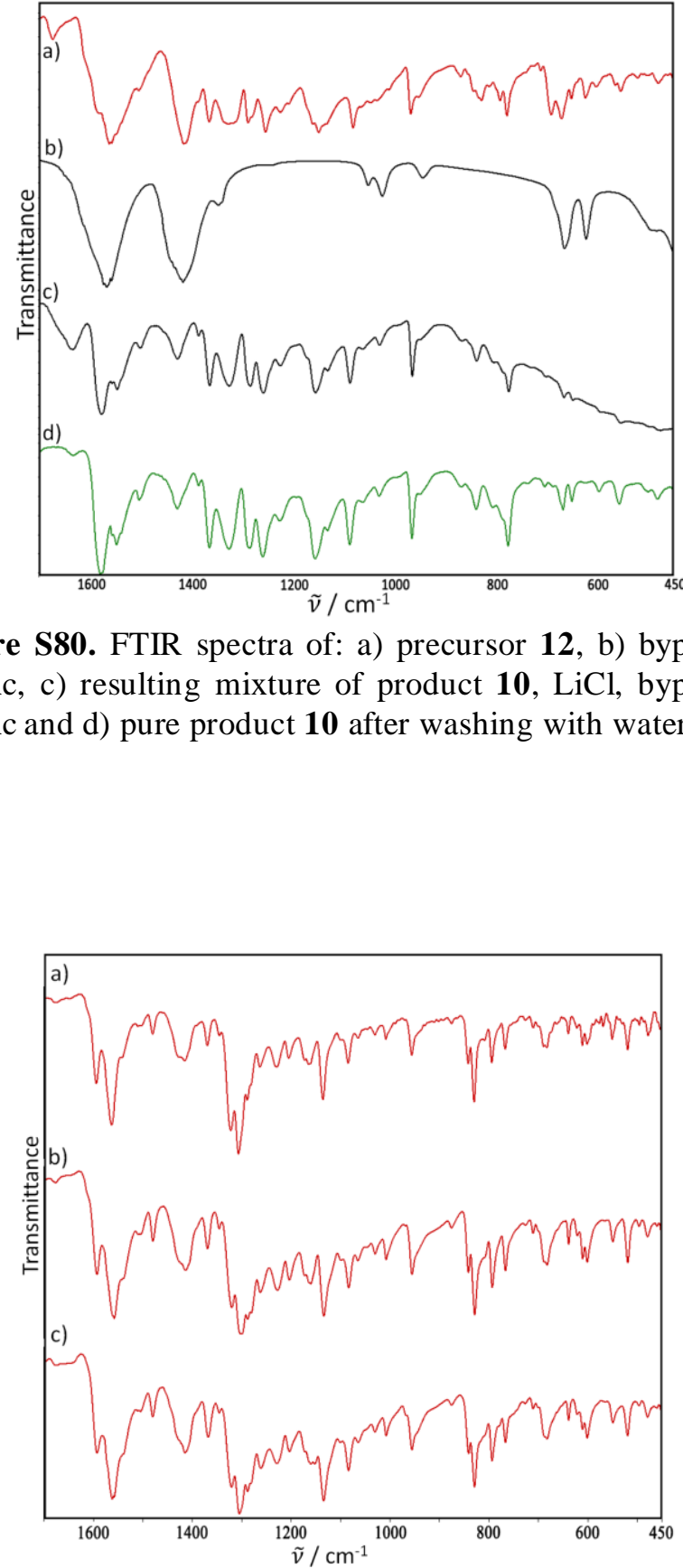

Figure S82. FTIR spectra of purified product 4 obtained by ion exchange reaction with $\mathrm{NaOAc}$ from: a) 2, b) 6 and c) 8 . 


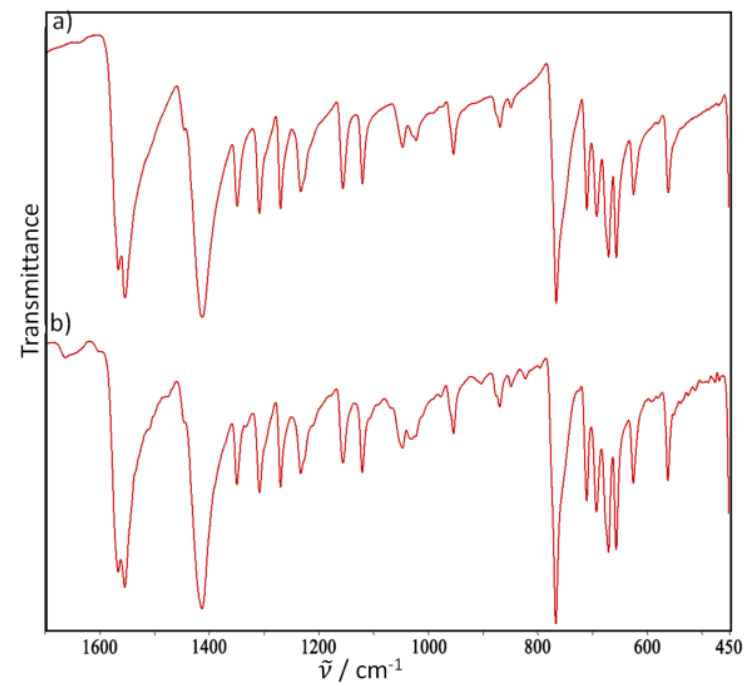

Figure S83. FTIR spectra of purified product 11 obtained by ion exchange reaction with $\mathrm{NaOAc}$ from: a) $\mathbf{9}$ and b) 13.

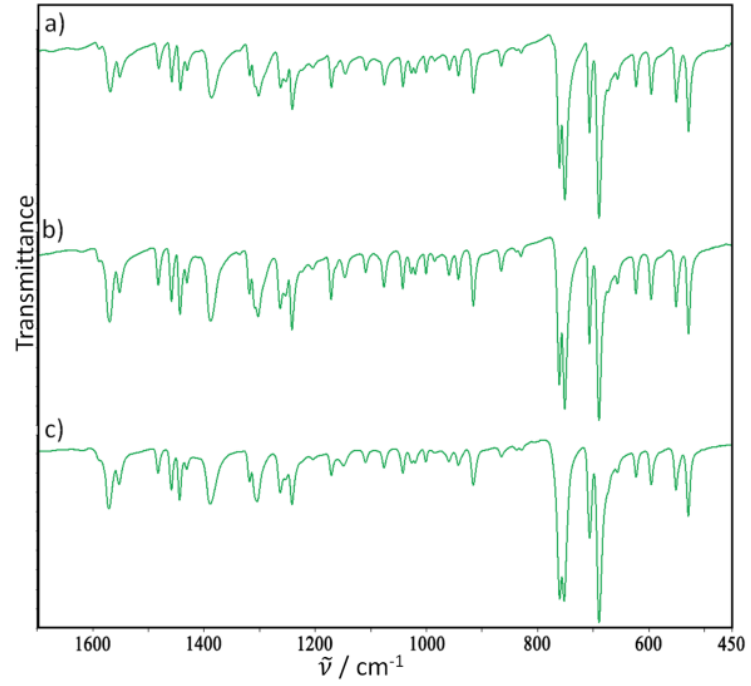

Figure S85. FTIR spectra of purified monopalladated product 5 obtained by ion exchange reaction with $\mathrm{LiCl}$ from: a) 1, b) 3 and c) 7 .

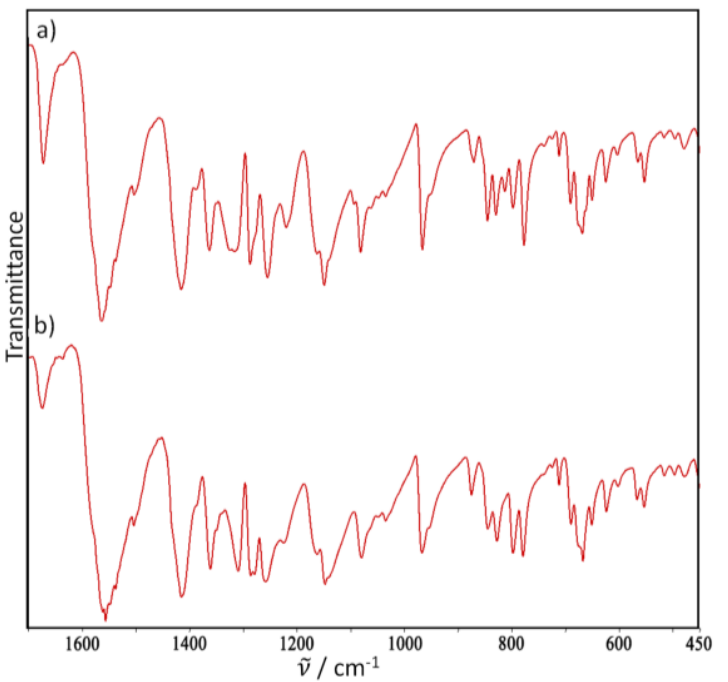

Figure S84. FTIR spectra of purified product 12 obtained by ion exchange reaction with $\mathrm{NaOAc}$ from: a) 10 and b) 14 .

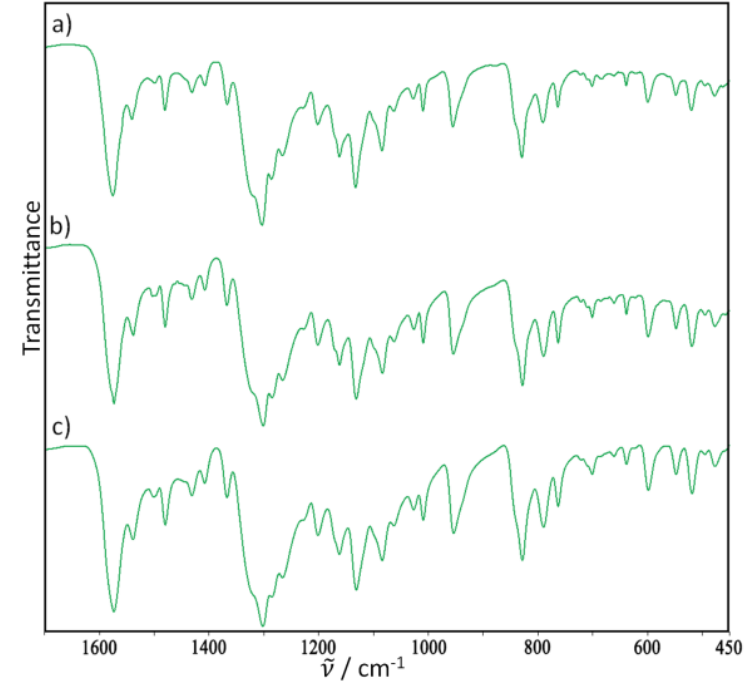

Figure S86. FTIR spectra of purified monopalladated product 6 obtained by ion exchange reaction with $\mathrm{LiCl}$ from: a) 2, b) 4 and c) 8 . 


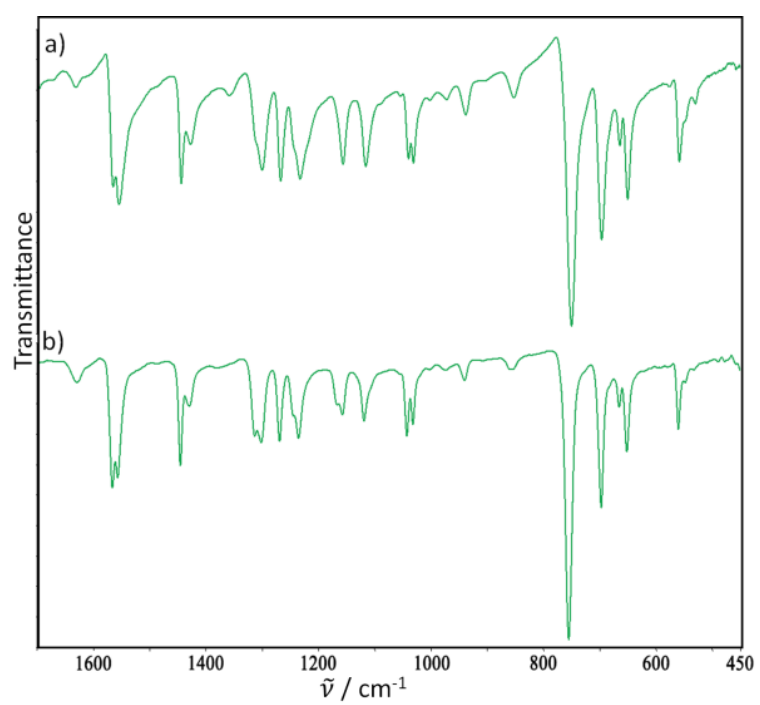

FigureS 87. FTIR spectra of purified dipalladated product $\mathbf{9}$ obtained by ion exchange reaction with $\mathrm{LiCl}$ from: a) $\mathbf{1 1}$ and b) 13.

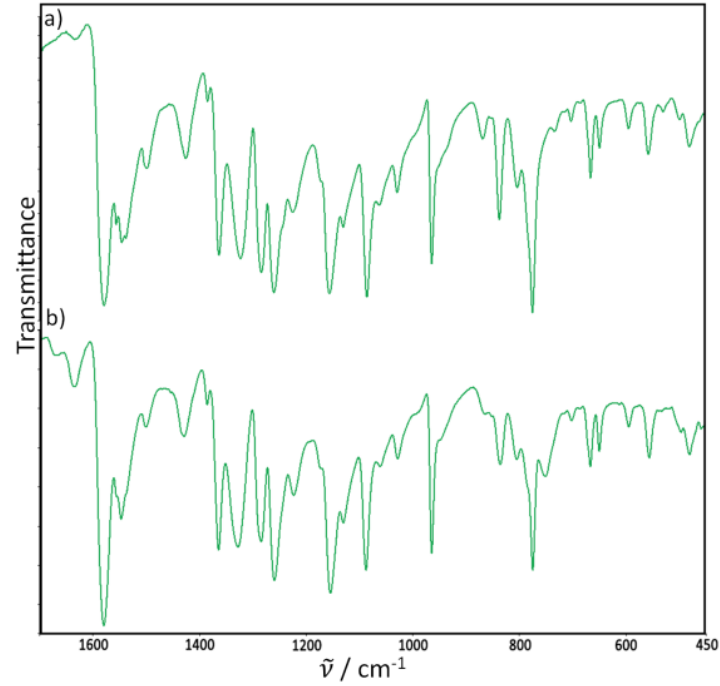

FigureS 88. FTIR spectra of purified dipalladated product 10 obtained by ion exchange reaction with $\mathrm{LiCl}$ from: a) $\mathbf{1 2}$ and b) 14 . 


\section{POWDER X-RAY DIFFRACTION}

\section{Structure solution from powder diffraction data.}

General remarks. Crystal structures of $\mathbf{7}$ and $\mathbf{1 3}$ were solved from powder diffraction data collected on a Panalytical Aeris laboratory powder diffractometer using Ni-filtered radiation from a copper anode. Sample was prepared in a thin layer on a silicon zero-background holder and data were collected in Bragg-Brentano geometry. Indexing was perfomed on selected peaks, positions of which were fitted by general peak fitting. Structure solution for both $\mathrm{C} 1 \mathrm{ABacac}$ and $\mathrm{C} 2 \mathrm{ABacac}$ was accomplished in direct space by treting expected molecular fragments as rigid bodies. All calculations were perfomerd using the program Topas (version 4.2) from Bruker-AXS (Karlsruhe, Germany).

Structure solution of 7. For 7 we have first taken the acetylacetonate and the monopalladated L1 moieties as separate rigid bodies. Structure solution was found only when preferred orientation was introduced into global optimisation in parallel. It was modelled using spherical harmonics coefficients of which were reset to zero before starting new optimisation cycles. Simulated annealing runs have positioned the acetylacetonate coordinated to $\mathrm{Pd}$ and even the trans influence of the carbon atom was evident since the Pd-O distance with carbon opposite was longer than the $\mathrm{Pd}-\mathrm{O}$ distance with nitrogen opposite. The acetylacetonate fragment was slightly tilted relative to the plane of the palladacycle. Geometry of the moiety as a whole was then optimised in vacuum and was introduced for the final Rietveld refinement cycles. The acetylacetonate fragment was allowed to rotate around the axis defined by two oxygen atoms and the free phenyl ring of $\mathbf{L} \mathbf{1}$ was also allowed to rotate. The final Rietveld fit exhibits serious mismatch between calculated and measured patterns which we attribute to inadequate modelling of preferred orientation. Several short contacts can be recognised involving hydrogen atoms which however originate from longer $\mathrm{C}-\mathrm{H}$ bonds as the rigid body was optimised in vacuum. For example, $\mathrm{C}-\mathrm{H}$ bond distances are typically around $1.06 \AA$ in our structure model whereas the usual $\mathrm{C}-\mathrm{H}$ distance from $\mathrm{X}$-ray diffraction will be around $0.95 \AA$.

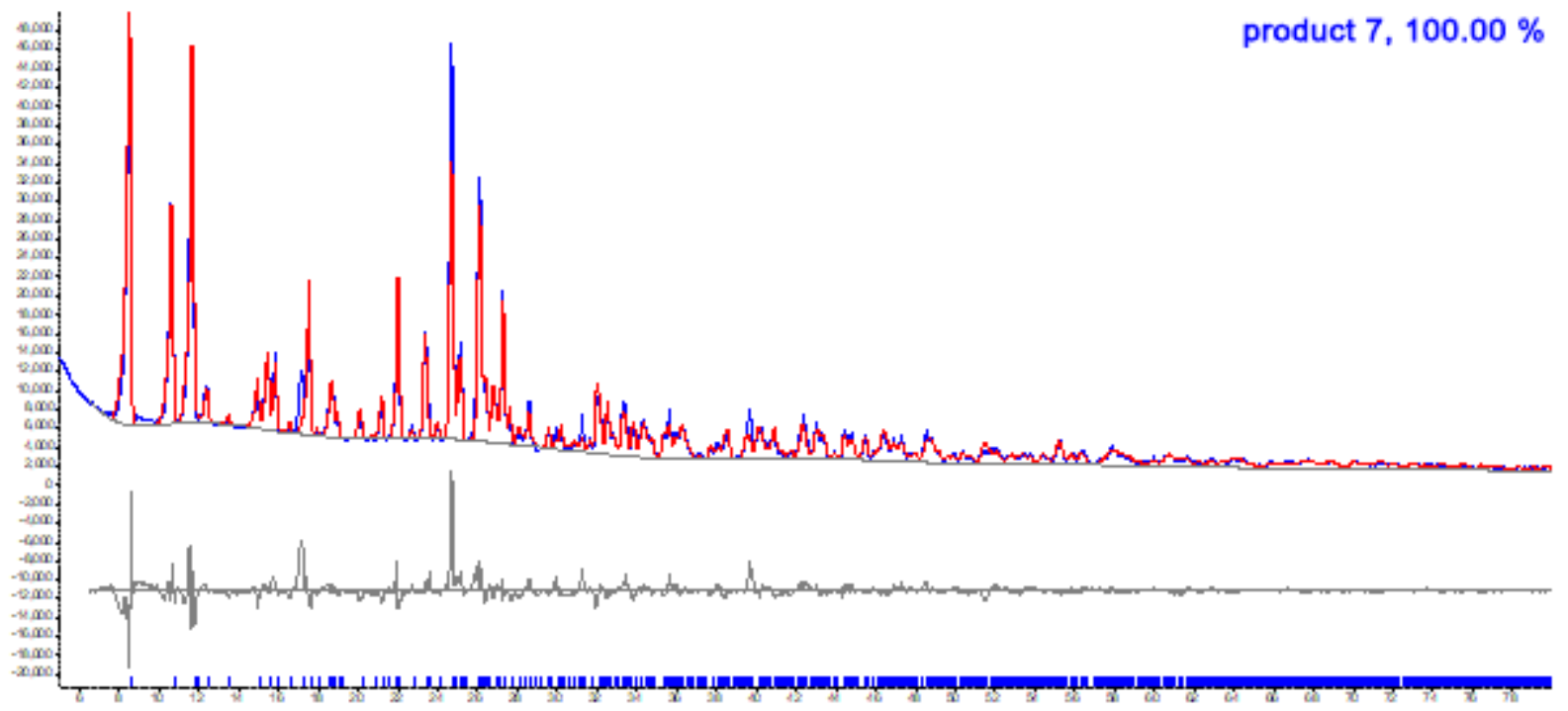

Figure S89. Rietveld plot of 7. Pattern collected in Bragg-Brenatano geometry using Nifiltered copper radiation. Blue-measured, red-calculated, grey-difference. Tick marks represent calculated peak position. Preferred orientation accounted for using spherical 
harmonics of the $6^{\text {th }}$ order. $a=13.983(1) \AA, b=10.629(1) \AA, c=11.426(1) \AA, \alpha=90^{\circ}, \beta=$ 111.303(4) $)^{\circ}, \gamma=90^{\circ}, V=1582.1(2) \AA^{3}$, space group: $P 21 / n$.

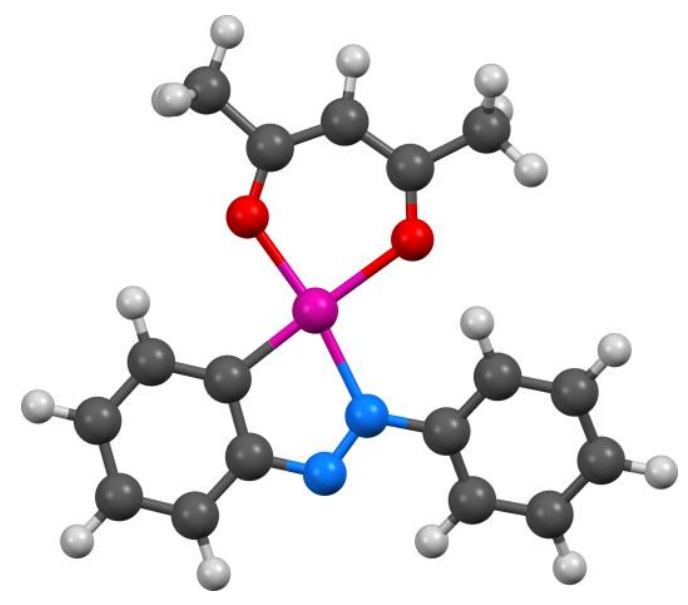

Figure S90. View of the molecular structure of 7. Palladium-purple, oxygen-red, nitrogenblue, carbon-grey, hydrogen-white.

Structure solution of $\mathbf{1 3}$. For 13, indexing afforded triclinic unit cell of approximately 540 $\AA^{3}$ which neatly fits one molecule of $\mathbf{1 3}$. In the space group P-1, half of the molecule must comprise the asymmetric unit and the centre of inversion is bisecting the $\mathrm{N}-\mathrm{N}$ bond. Structure solution was attempted using half of the molecule, previously optimised in vacuum, as a rigid body. A dummy atom was positioned to bisect the N-N bond and the rigid body was positioned with this dummy atom lying on a centre of inversion at $(0,0,0)$. This meant that only the orientation of the rigid body needed to be found. Preferred orientation was modelled using spherical harmonics.

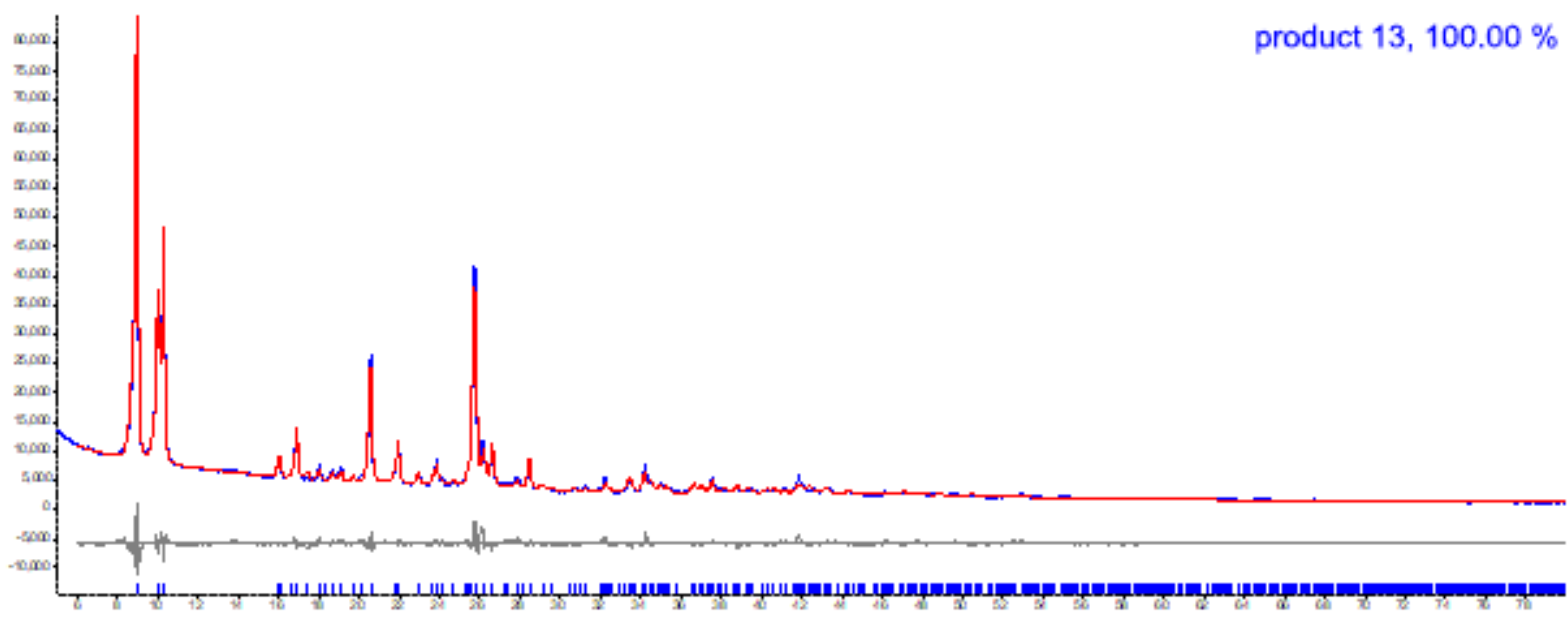

Figure S91. Rietveld plot of 13. Pattern collected in Bragg-Brenatano geometry using Nifiltered copper radiation. Blue-measured, red-calculated, grey-difference. Tick marks represent calculated peak position. Preferred orientation accounted for using spherical harmonics of the $6^{\text {th }}$ order. $a=5.6613(4) \AA, b=11.3428(9) \AA, c=9.7569(8) \AA, \alpha=$ 111.274(5) $)^{\circ}, \beta=96.289(5)^{\circ}, \gamma=107.464(6)^{\circ}, V=539.86(8) \AA^{3}$, space group: $P-1$. 


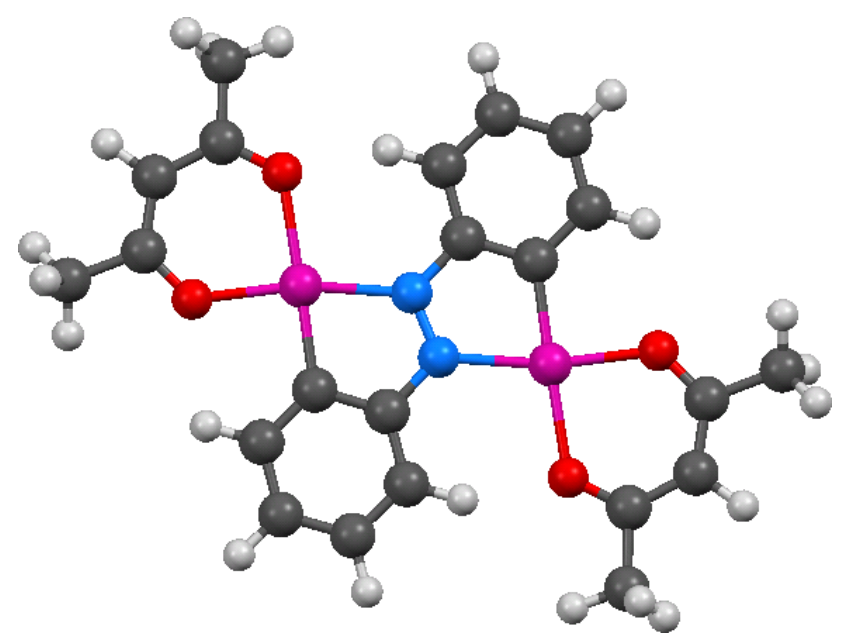

Figure S92. View of the molecular structure of 13. Palladium-purple, oxygen-red, nitrogenblue, carbon-grey, hydrogen-white.

\section{PXRD OF REACTANTS AND PRODUCT}

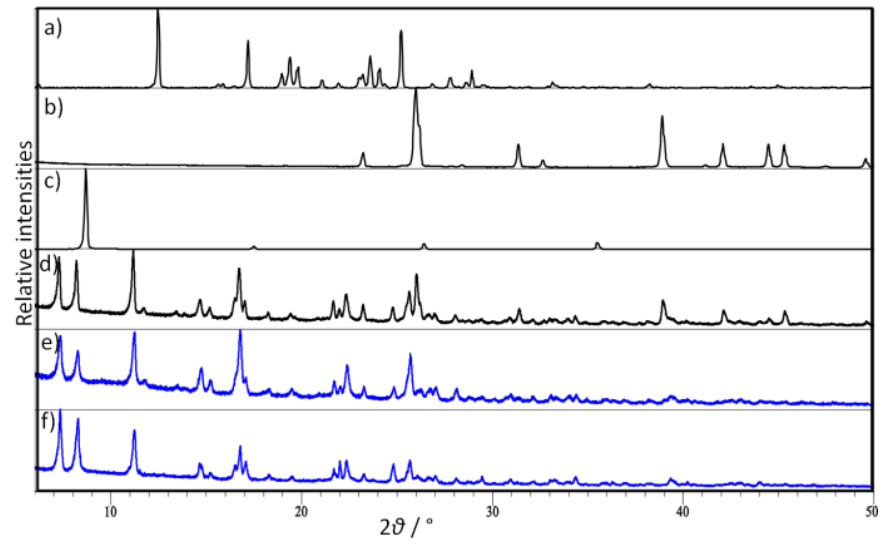

Figure S93. PXRD patterns of: a) $\mathbf{L 1}$, b) $\mathrm{NaBF}_{4}$, c) $\mathrm{NaOAc}, \mathrm{d})$ crude product 1 prepared by IAG using $\mathrm{NaOAc}$ and $\mathrm{NaBF}_{4}$ as additives, e) pure product 1 after washing with water and f) product $\mathbf{1}$ recrystallized from $\mathrm{MeCN}$.

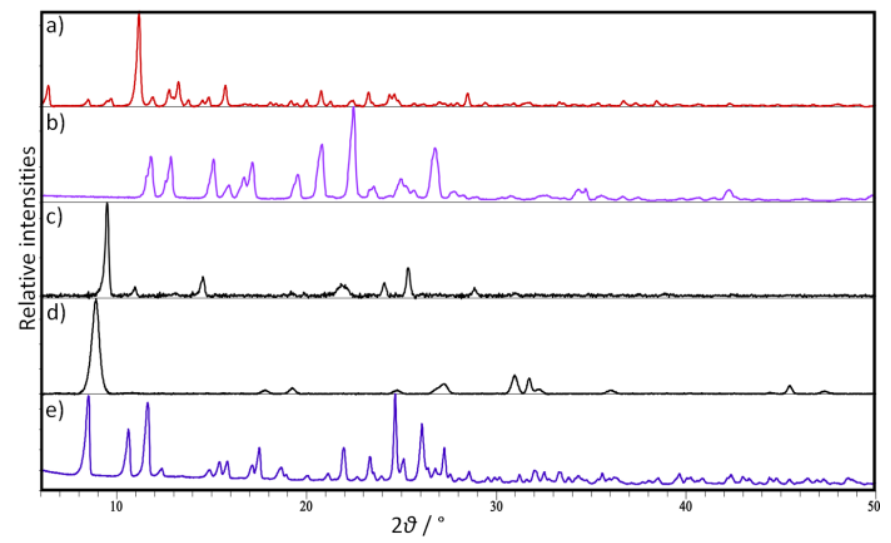

Figure S95. PXRD patterns of: a) precursor 3, b) product $7, \mathrm{Li}(\mathrm{acac}), \mathrm{LiOAc}$ and e) pure product 7 after washing with water.

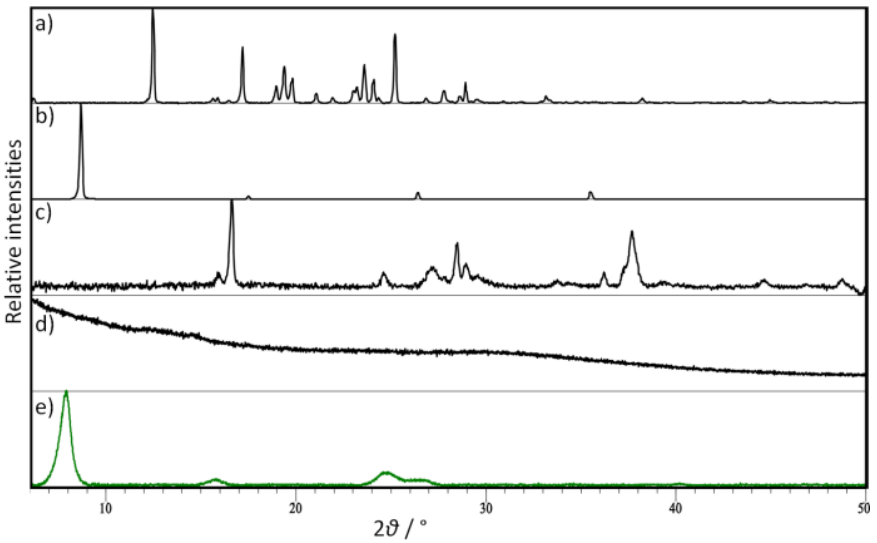

Figure S94. PXRD patterns of: a) L1, b) $\mathrm{NaOAc}$, c) $\mathrm{PdCl}_{2}$, d) crude product 9 prepared by IAG using $\mathrm{NaOAc}$ as additive and e) pure product 9 after washing with water.

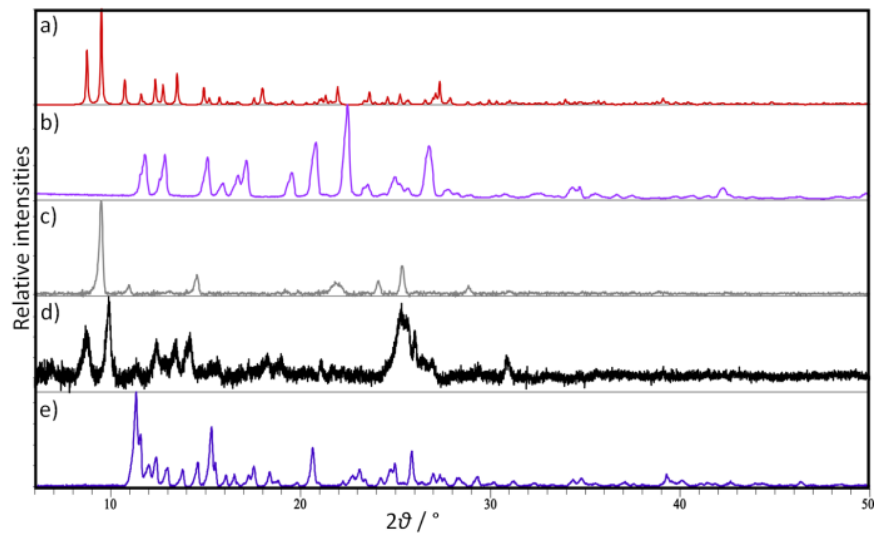

Figure S96. PXRD patterns of: a) precursor 4, b) $\mathrm{Li}(\mathrm{acac}), \mathrm{c})$ byproduct $\mathrm{LiOAc}$, d) resulting mixture of product $\mathbf{8}, \mathrm{Li}(\mathrm{acac}), \mathrm{LiOAc}$ and e) pure product $\mathbf{8}$ after washing with water. 


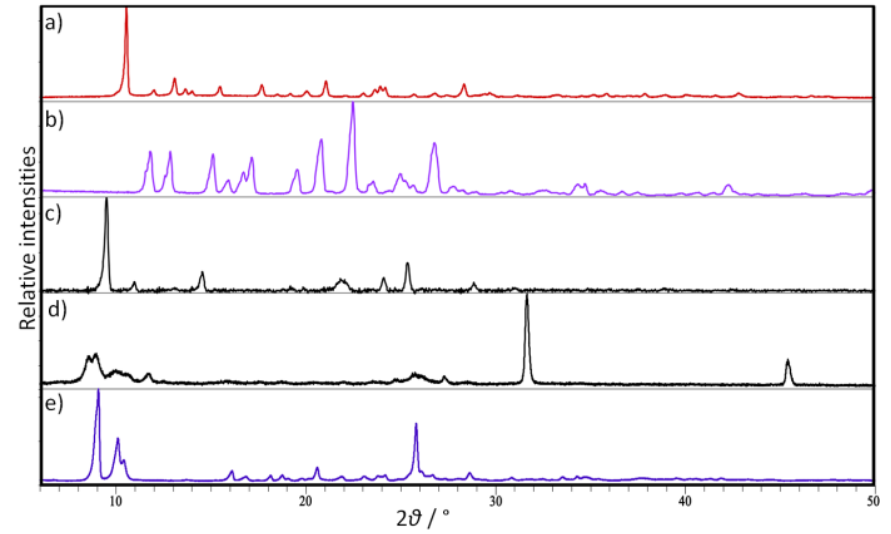

Figure S97. PXRD patterns of: a) precursor 11, b) $\mathrm{Li}(\mathrm{acac}), \mathrm{c})$ byproduct $\mathrm{LiOAc}$, d) resulting mixture of product 13, Li(acac), LiOAc and e) pure product 13 after washing with water.

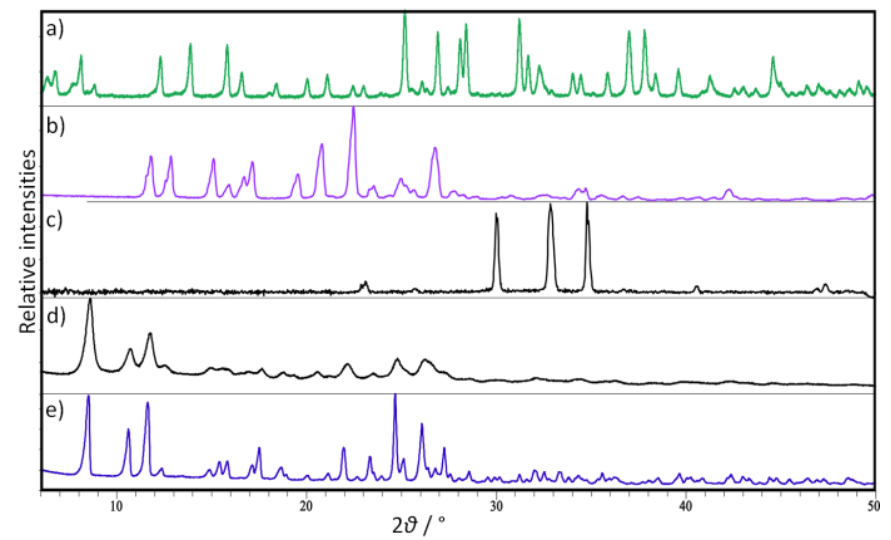

Figure S99. PXRD patterns of: a) precursor 5, b) $\mathrm{Li}(\mathrm{acac}), \mathrm{c})$ byproduct $\mathrm{LiCl}, \mathrm{d})$ resulting mixture of product 7, $\mathrm{Li}(\mathrm{acac}), \mathrm{LiCl}$ and e) pure product 7 after washing with water.

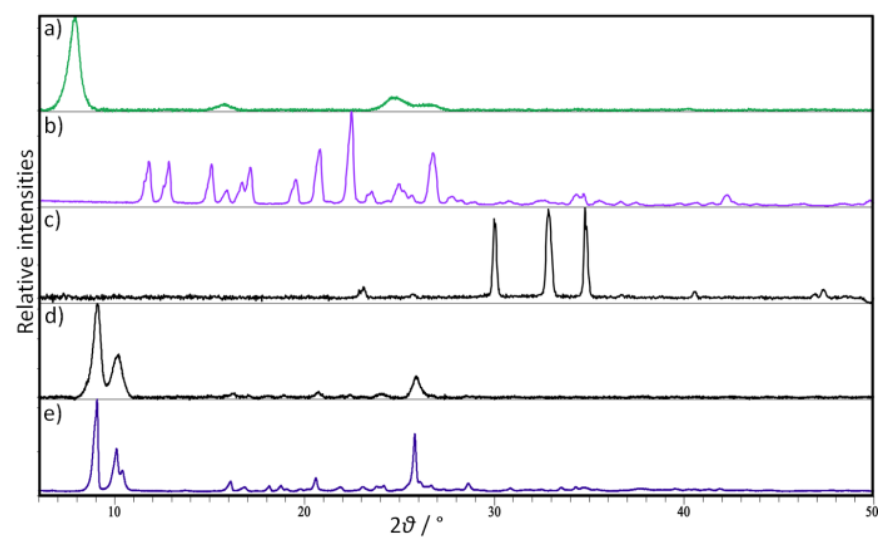

Figure S101. PXRD patterns of: a) precursor 9, b) $\mathrm{Li}(\mathrm{acac})$, c) byproduct $\mathrm{LiCl}$, d) resulting mixture of $\mathbf{1 3}$, $\mathrm{Li}(\mathrm{acac}), \mathrm{LiCl}$ and d) pure product $\mathbf{1 3}$ after washing with water.

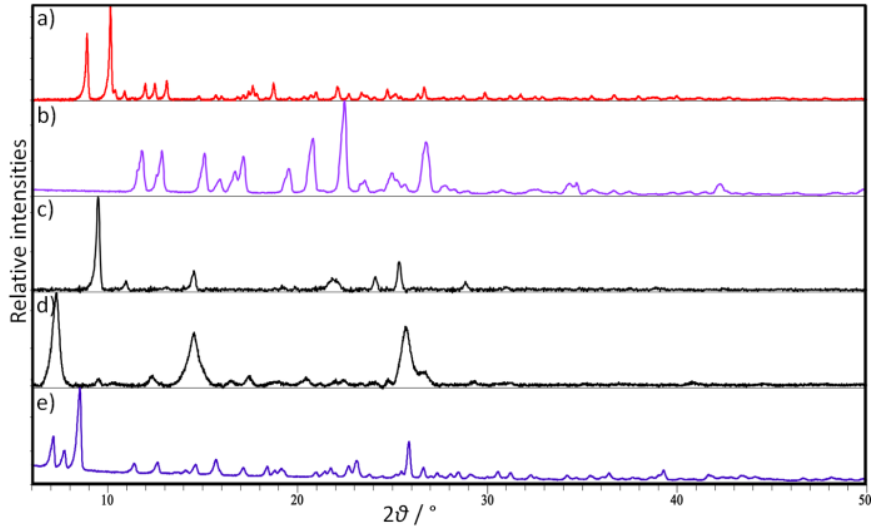

Figure S98. PXRD patterns of: a) precursor 12, b) $\mathrm{Li}(\mathrm{acac})$, c) byproduct LiOAc, d) resulting mixture of product $\mathbf{1 4}, \mathrm{Li}(\mathrm{acac}), \mathrm{LiOAc}$ and e) pure dipalladated $\mathbf{1 4}$ after washing with water.

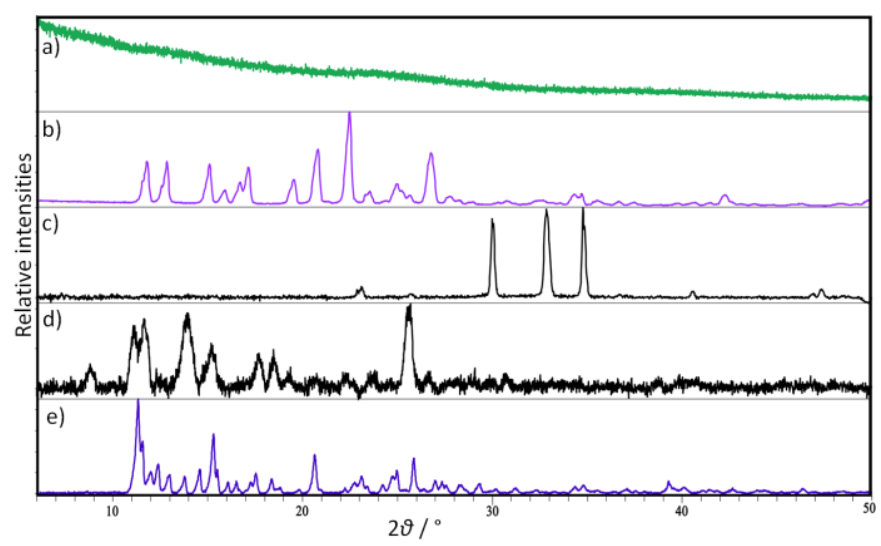

Figure S100. PXRD patterns of: a) precursor 6 , b) $\mathrm{Li}(\mathrm{acac}), \mathrm{c})$ byproduct $\mathrm{LiCl}, \mathrm{d}$ ) resulting mixture of product $\mathbf{8}, \mathrm{Li}(\mathrm{acac}), \mathrm{LiCl}$ and e) pure product $\mathbf{8}$ after washing with water.

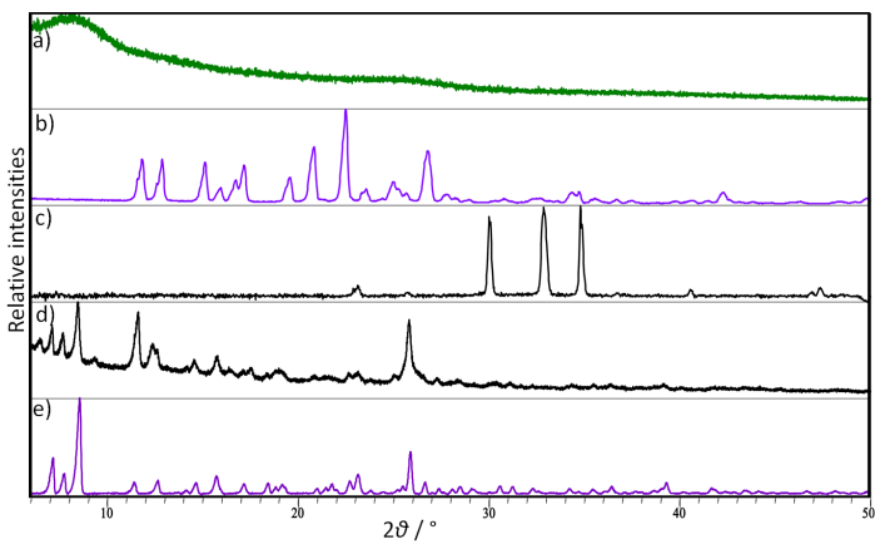

Figure S102. PXRD patterns of: a) precursor 10, b) $\mathrm{Li}(\mathrm{acac}), \mathrm{c})$ byproduct $\mathrm{LiCl}, \mathrm{d})$ resulting mixture of product 14, $\mathrm{Li}(\mathrm{acac}), \mathrm{LiCl}$ and d) pure product 14 after washing with water. 


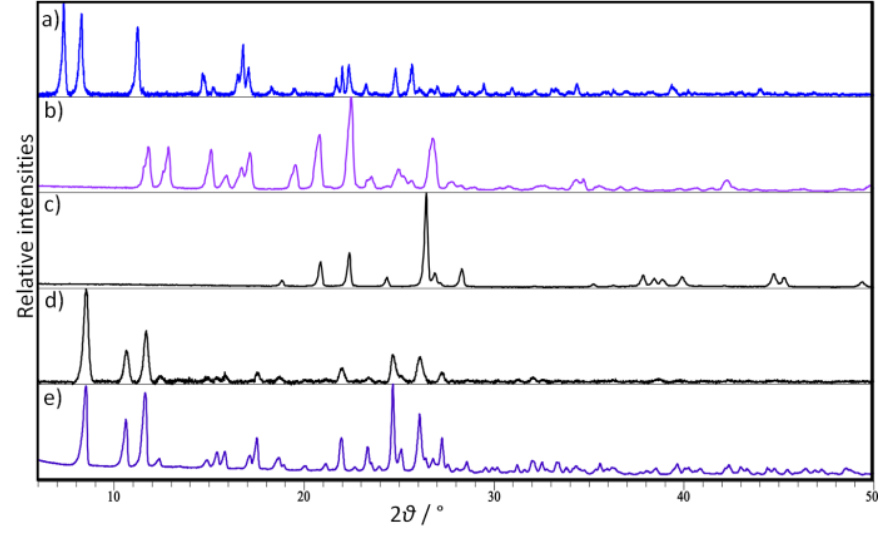

Figure S103. PXRD patterns of: a) precursor 1, b) $\mathrm{Li}(\mathrm{acac})$, c) byproduct $\mathrm{LiBF}_{4}$ d) resulting mixture of $\mathbf{7}$, $\mathrm{Li}(\mathrm{acac}), \mathrm{LiBF}_{4}$ and e) pure product 7 after washing with water.

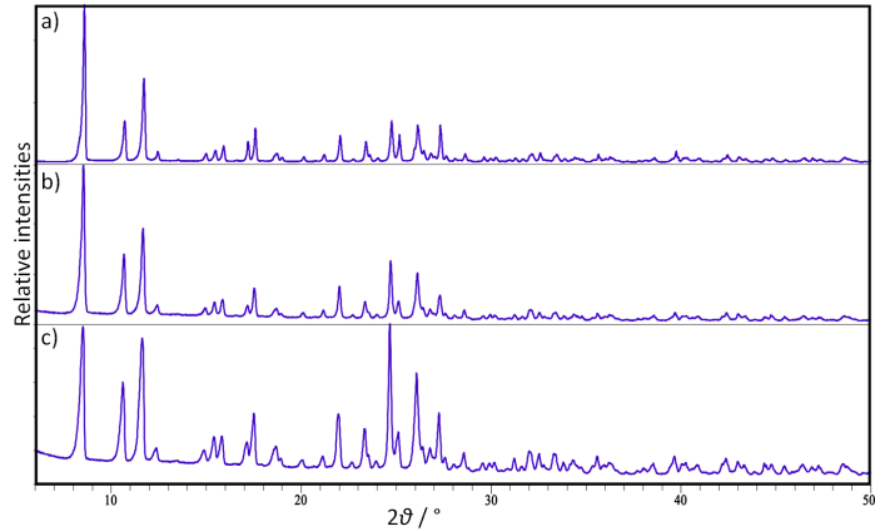

Figure S105. PXRD patterns of purified product 7 obtained by ion exchange reaction with $\operatorname{Li}(\mathrm{acac})$ from: a) $\mathbf{1}$, b) $\mathbf{3}$ and c) 5 .

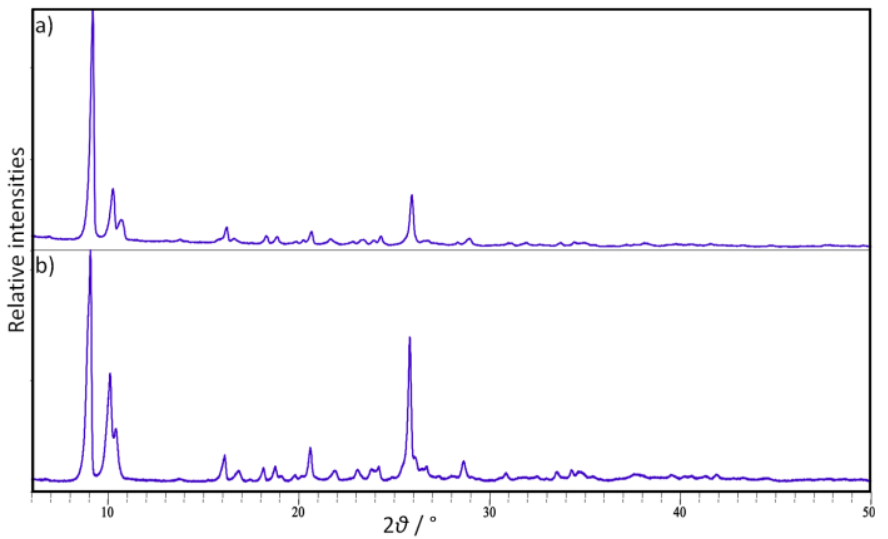

Figure S107. PXRD patterns of purified product 13 obtained by ion exchange reaction with $\operatorname{Li}(\mathrm{acac})$ from: a) 9 and b) $\mathbf{1 1}$.

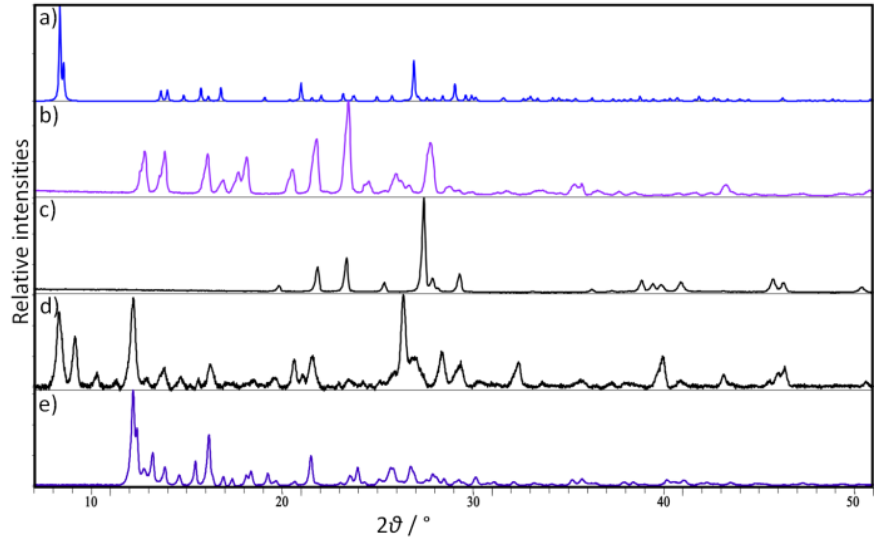

Figure S104. PXRD patterns of: a) precursor 2, b) $\mathrm{Li}(\mathrm{acac})$, c) byproduct $\mathrm{LiBF}_{4}$, d) resulting mixture of product $\mathbf{8}, \mathrm{Li}\left(\right.$ acac), $\mathrm{LiBF}_{4}$ and e) pure product $\mathbf{8}$ after washing with water.

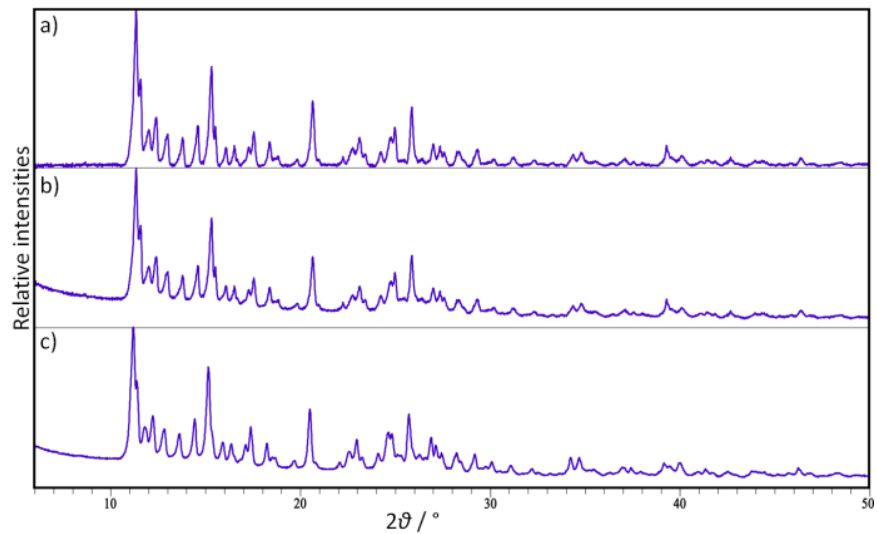

Figure S106. FTIR spectra of purified product 8 obtained by ion exchange reaction with $\operatorname{Li}(\mathrm{acac})$ from: a) 2 , b) 4 and c) 6 .

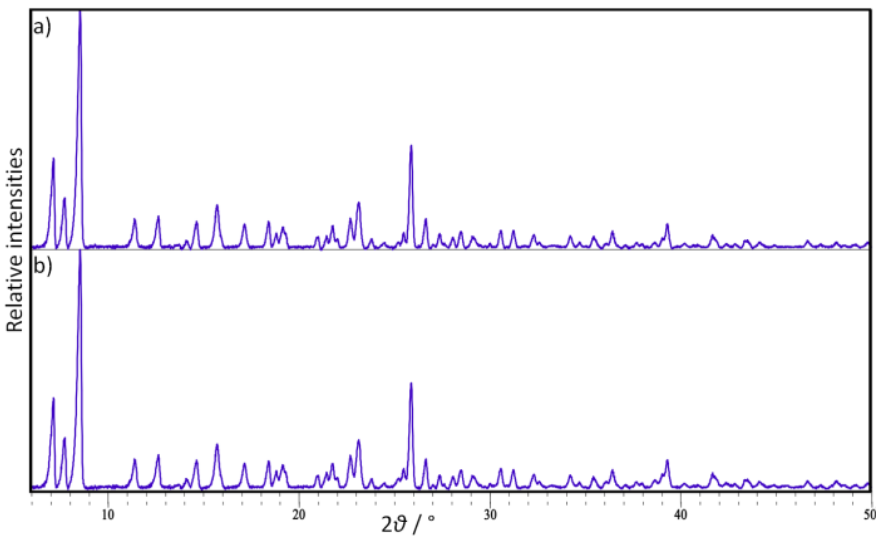

Figure S108. PXRD patterns of purified product 14 obtained by ion exchange reaction with $\operatorname{Li}(\mathrm{acac})$ from: a) 10 and b) 12. 


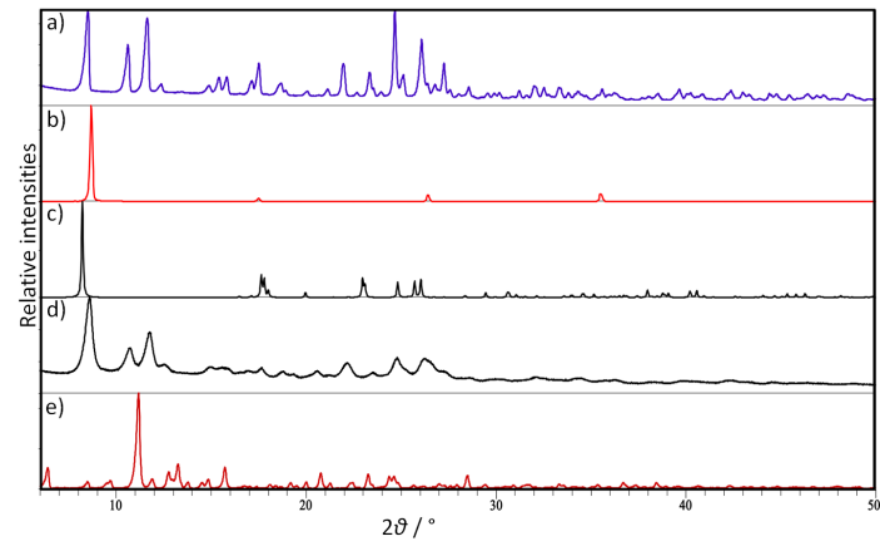

Figure S109 PXRD patterns of: a) precursor 7, b) $\mathrm{NaOAc}$, c) byproduct $\mathrm{Na}(\mathrm{acac}), \mathrm{d})$ resulting mixture of product $\mathbf{3}$, $\mathrm{NaOAc}, \mathrm{Na}(\mathrm{acac})$ and e) pure product 3 after washing with water

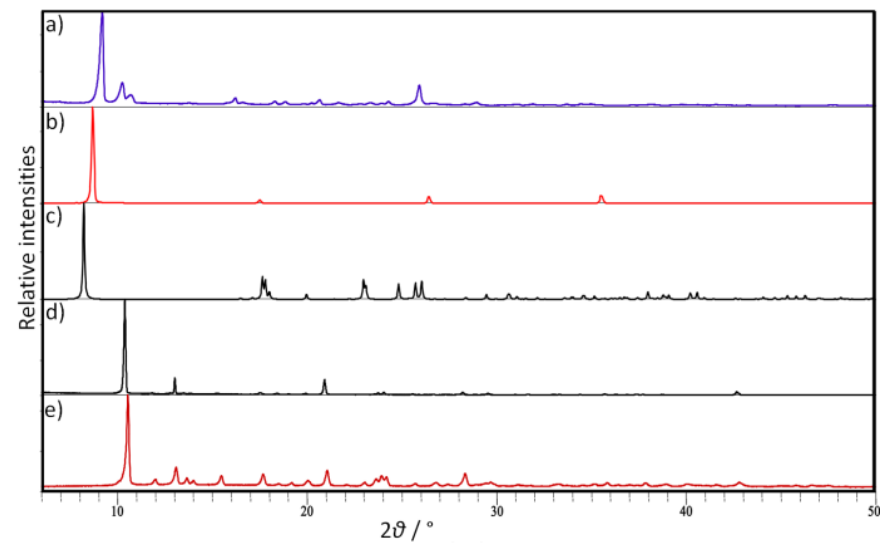

Figure S111. PXRD patterns of: a) precursor 13, b) $\mathrm{NaOAc}, \mathrm{c})$ byproduct $\mathrm{Na}(\mathrm{acac})$, d) resulting mixture of 11, $\mathrm{NaOAc}, \mathrm{Na}(\mathrm{acac})$ and e) pure product $\mathbf{1 1}$ after washing with water.

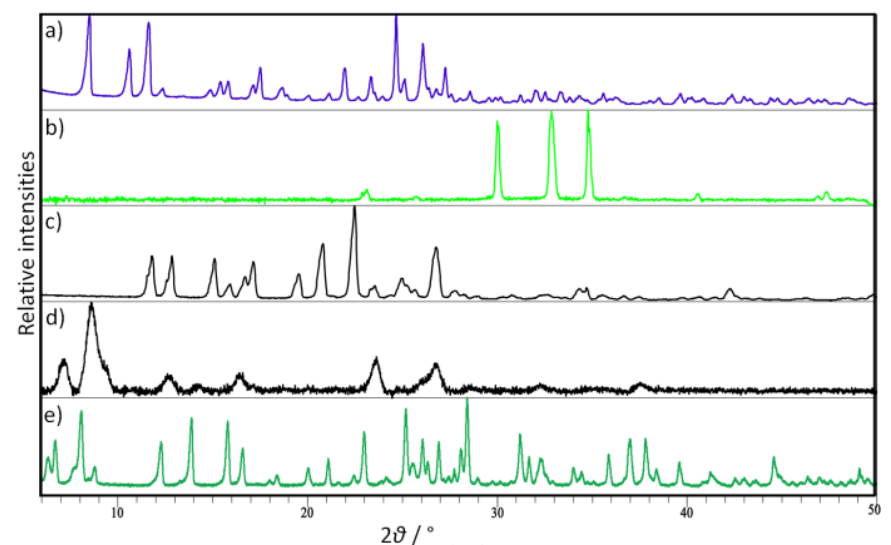

Figure S113. PXRD patterns of: a) precursor 7, b) $\mathrm{LiCl}$, c) byproduct $\mathrm{Li}(\mathrm{acac}), \mathrm{d})$ resulting mixture of $\mathbf{5}, \mathrm{LiCl}$, $\mathrm{Li}(\mathrm{acac})$ and e) pure product 5 after washing with water.

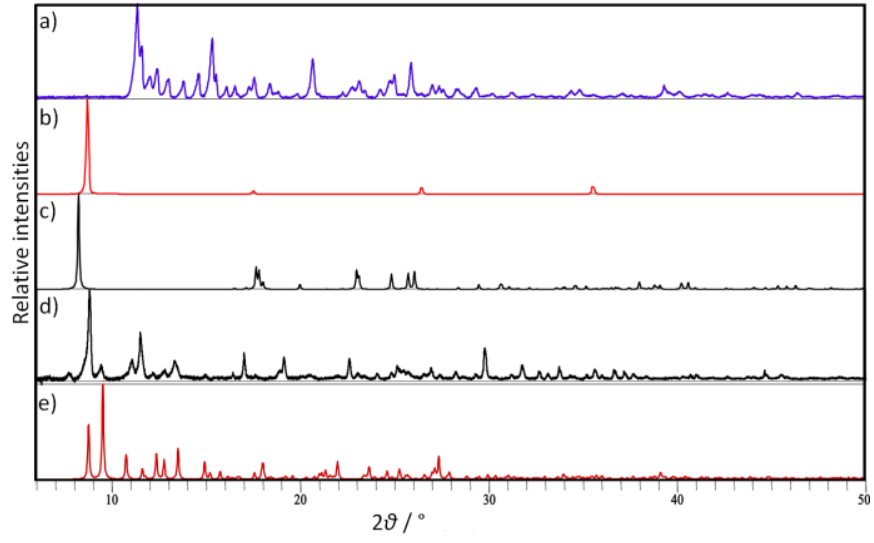

Figure S110. PXRD patterns of: a) initial precursor 8, b) $\mathrm{NaOAc}$, c) byproduct $\mathrm{Na}(\mathrm{acac})$, d) resulting mixture of product $4, \mathrm{NaOAc}, \mathrm{Na}(\mathrm{acac})$ and e) pure product 4 after washing with water.

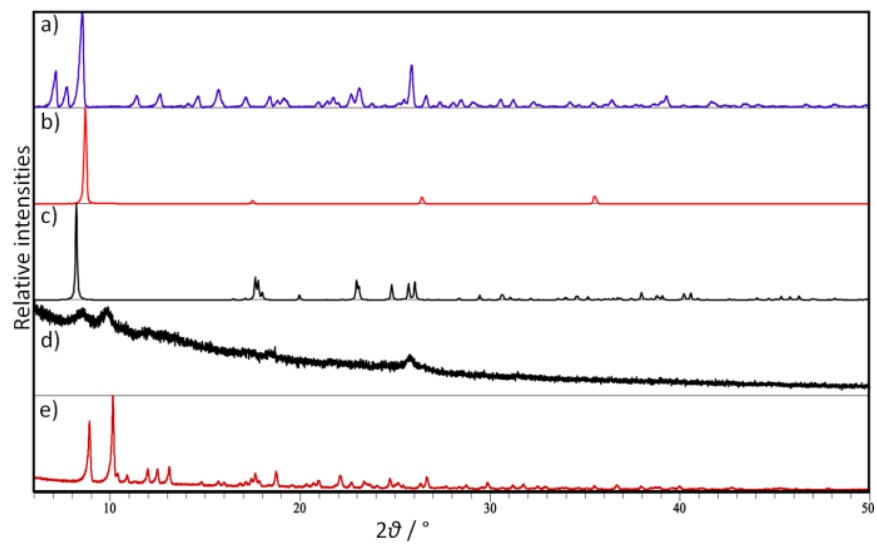

Figure S112. PXRD patterns of: a) precursor 14, b) $\mathrm{NaOAc}$, c) byproduct $\mathrm{Na}(\mathrm{acac})$, d) resulting mixture of product 12, $\mathrm{NaOAc}, \mathrm{Na}(\mathrm{acac})$ and e) pure product 12 after washing with water.

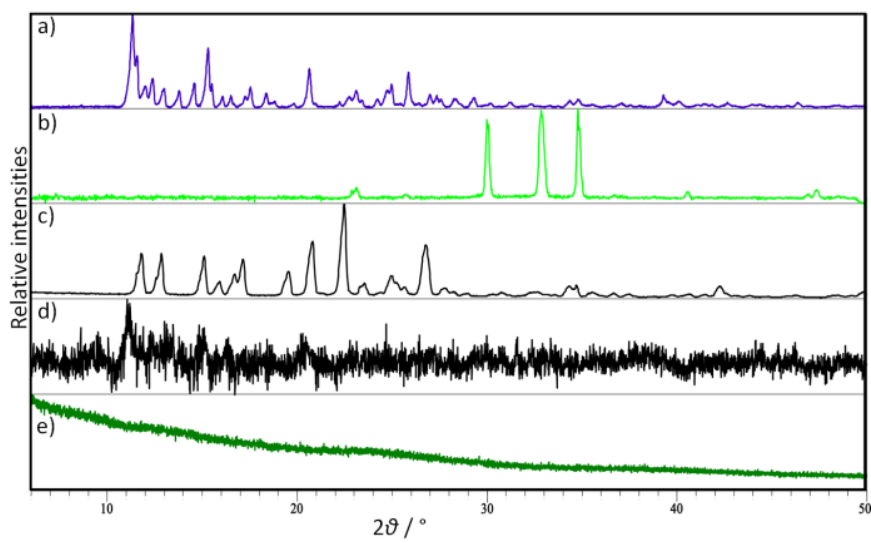

Figure S114. PXRD patterns of: a) precursor 8, b) $\mathrm{LiCl}$, c) byproduct $\mathrm{Li}(\mathrm{acac}), \mathrm{d})$ resulting mixture of $\mathbf{6}, \mathrm{LiCl}$, $\mathrm{Li}(\mathrm{acac})$ and e) pure product 6 after washing with water. 


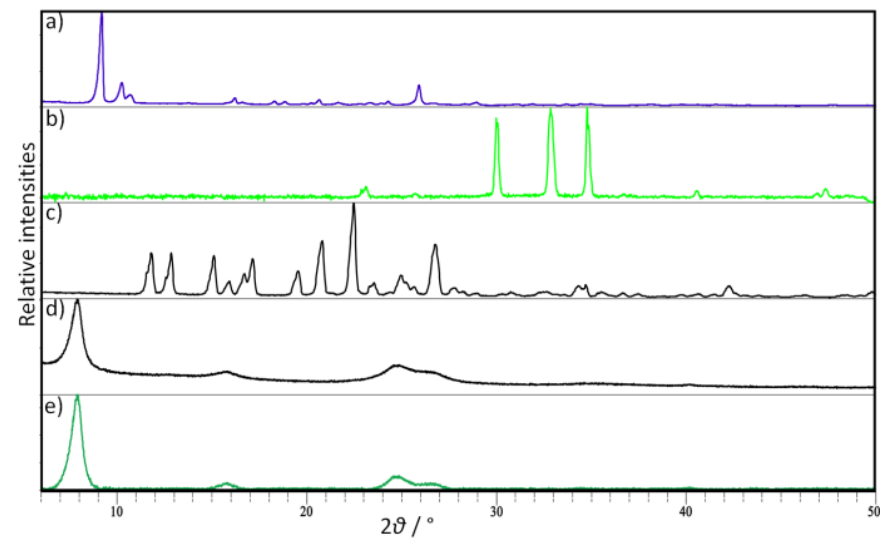

Figure S115. PXRD patterns of: a) precursor 13, b) $\mathrm{LiCl}$, c) byproduct $\mathrm{Li}(\mathrm{acac}), \mathrm{d})$ resulting mixture of product $\mathbf{9}$, $\mathrm{LiCl}, \mathrm{Li}(\mathrm{acac})$ and e) pure product 9 after washing with water.

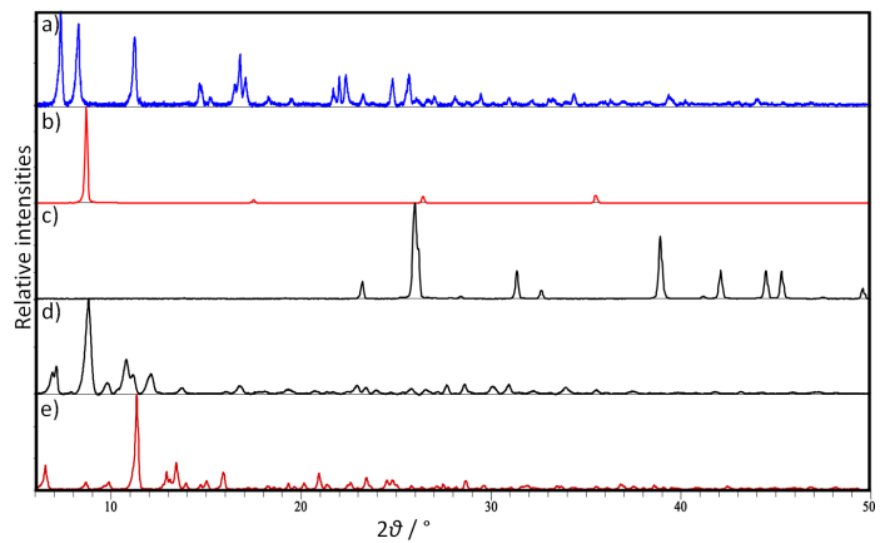

Figure S117. PXRD patterns of: a) precursor 1, b) $\mathrm{NaOAc}$, c) byproduct $\mathrm{NaBF}_{4}$, d) resulting mixture of product 3, NaOAc, $\mathrm{NaBF}_{4}$ and e) pure product $\mathbf{3}$ after washing with water.

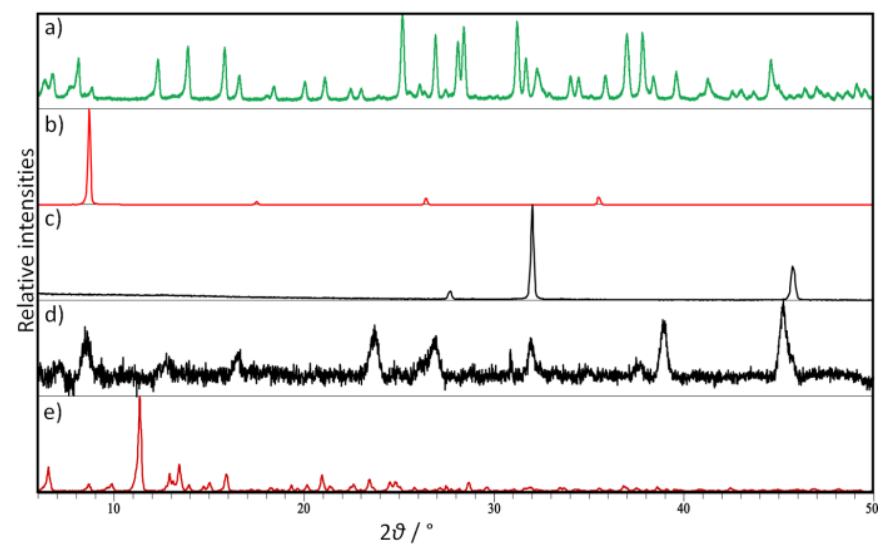

Figure S119. PXRD patterns of: a) precursor 5, b) $\mathrm{NaOAc}, \mathrm{c})$ byproduct $\mathrm{NaCl}$, d) resulting mixture of product 3, $\mathrm{NaOAc}$, byproduct $\mathrm{NaCl}$ and e) pure product 3 after washing with water.

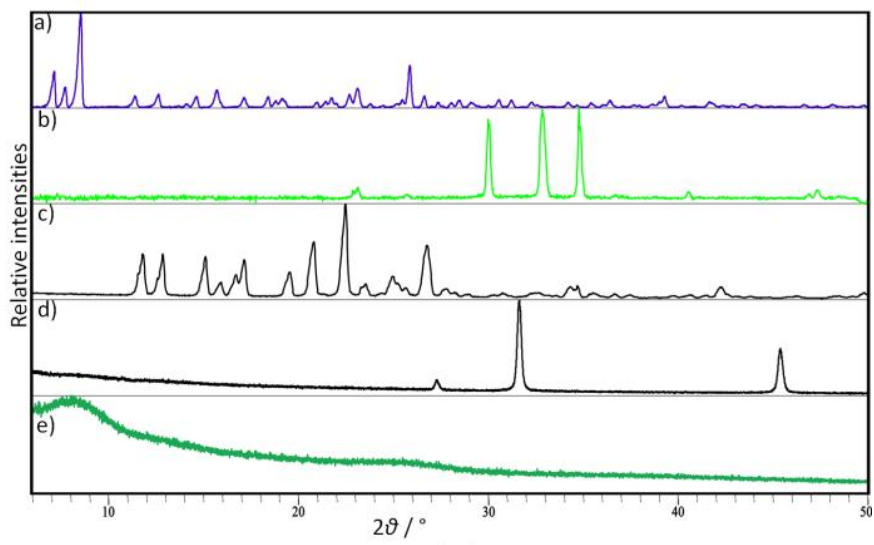

Figure S116. PXRD patterns of: a) precursor 14, b) $\mathrm{LiCl}$, c) byproduct $\mathrm{Li}(\mathrm{acac}), \mathrm{d})$ resulting mixture of product $\mathbf{1 0}$, $\mathrm{LiCl}, \mathrm{Li}(\mathrm{acac})$ and e) pure product $\mathbf{1 0}$ after washing with water.

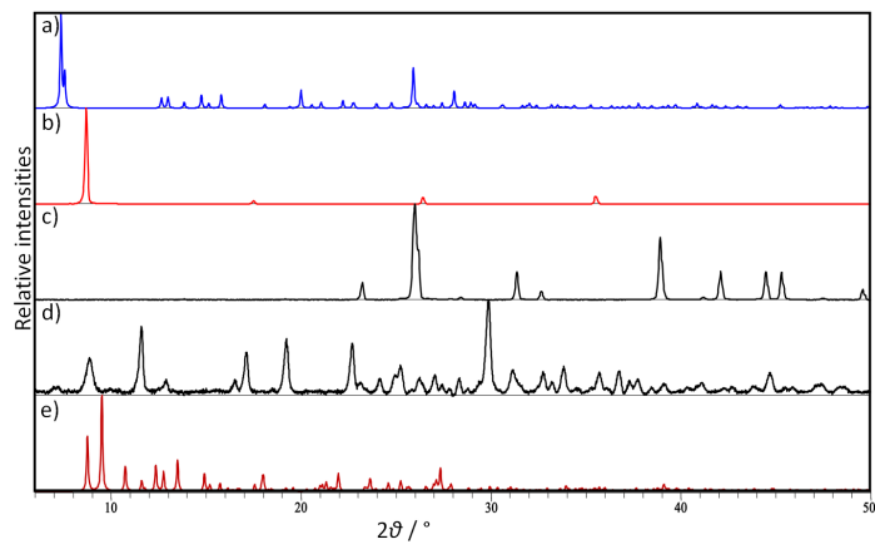

Figure S118. PXRD patterns of: a) precursor 2, b) $\mathrm{NaOAc}$, c) byproduct $\mathrm{NaBF}_{4}$, d) resulting mixture of product $4, \mathrm{NaOAc} \mathrm{NaBF}_{4}$ and e) pure product 4 after washing with water.

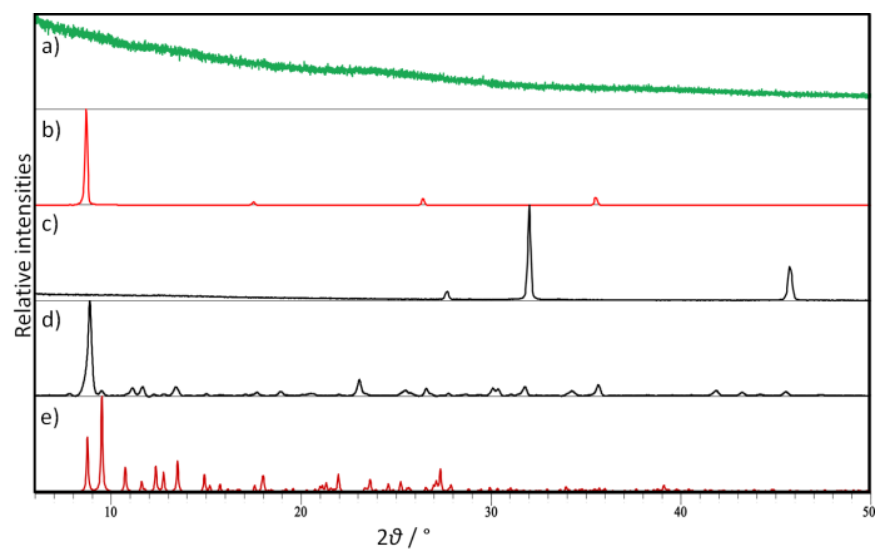

Figure S120. PXRD patterns of: a) 6, b) $\mathrm{NaOAc}$, c) byproduct $\mathrm{NaCl}, \mathrm{d}$ ) resulting mixture of product $4, \mathrm{NaOAc}$, byproduct $\mathrm{NaCl}$ and e) pure product 4 after washing with water. 


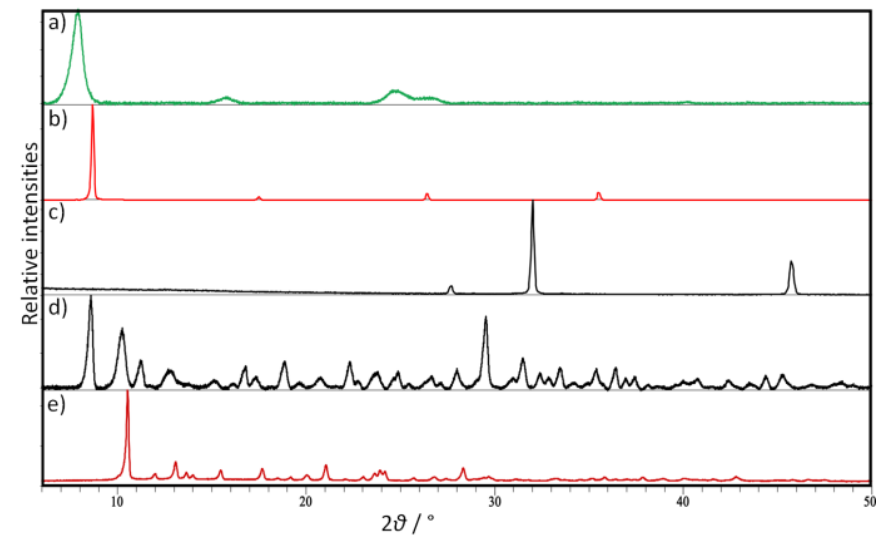

Figure S121. PXRD patterns of: a) precursor 9, b) $\mathrm{NaOAc}, \mathrm{c})$ byproduct $\mathrm{NaCl}, \mathrm{d}$ ) resulting mixture of product 11, $\mathrm{NaOAc}$, byproduct $\mathrm{NaCl}$ and e) pure product $\mathbf{1 1}$ after washing with water.

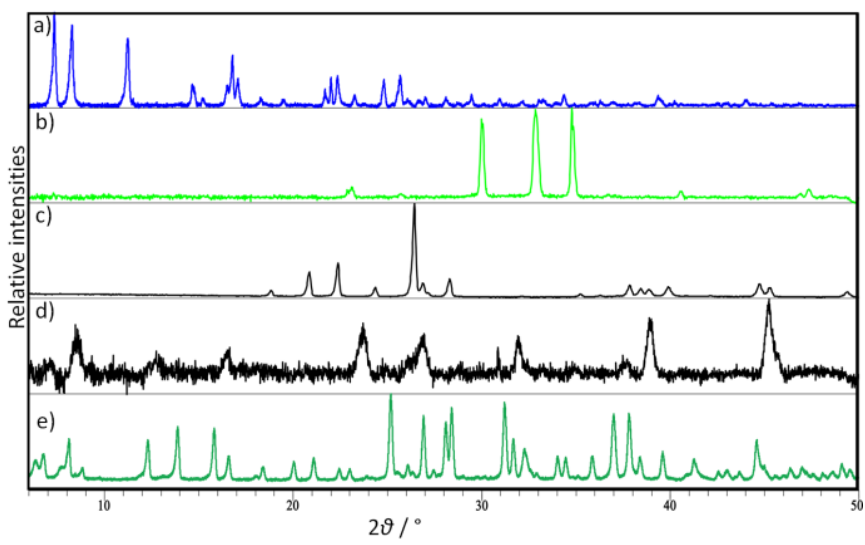

Figure S123. PXRD patterns of: a) precursor 1, b) $\mathrm{LiCl}$, c) byproduct $\mathrm{LiBF}_{4}, \mathrm{~d}$ ) resulting mixture of product $\mathbf{5}, \mathrm{LiCl}$, $\mathrm{LiBF}_{4}$ and e) pure product 5 after washing with water.

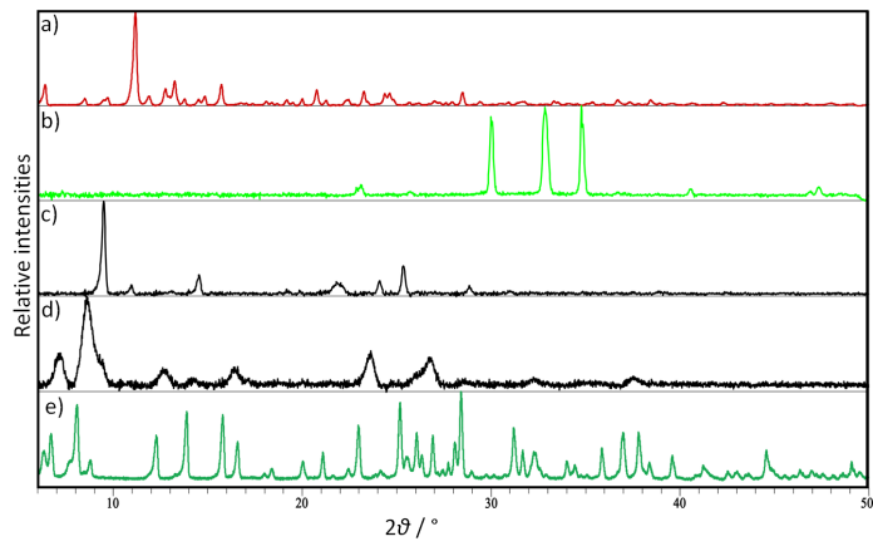

Figure S125. PXRD patterns of: a) precursor 3, b) $\mathrm{LiCl}$, c) byproduct $\mathrm{LiOAc}, \mathrm{d})$ resulting mixture of product $\mathbf{5}$, $\mathrm{LiCl}$, byproduct LiOAc and e) pure product 5 after washing with water.

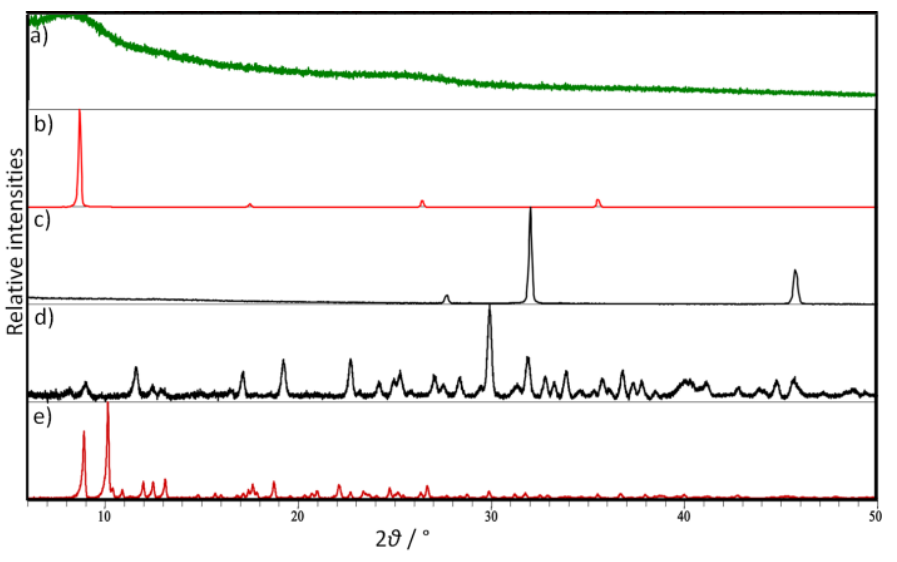

Figure S122. PXRD patterns of: a) precursor 10, b) $\mathrm{NaOAc}, \mathrm{c})$ byproduct $\mathrm{NaCl}, \mathrm{d}$ ) resulting mixture of product 12, $\mathrm{NaOAc}$, byproduct $\mathrm{NaCl}$ and e) pure product 12 after washing with water.

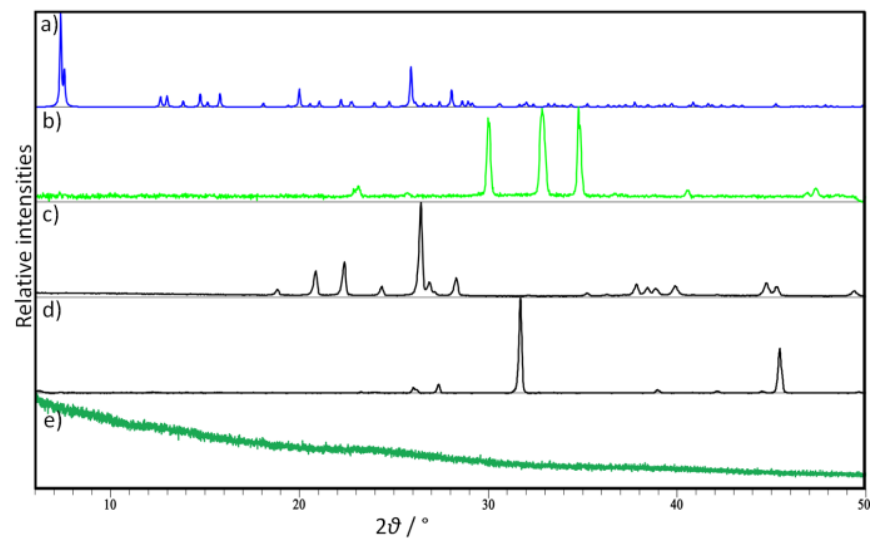

Figure S124. FTIR spectra of: a) precursor 2, b) $\mathrm{LiCl}, \mathrm{c}$ ) byproduct $\mathrm{LiBF}_{4}$, d) resulting mixture of product $\mathbf{6}, \mathrm{LiCl}$, $\mathrm{LiBF}_{4}$ and e) pure product 6 after washing with water.

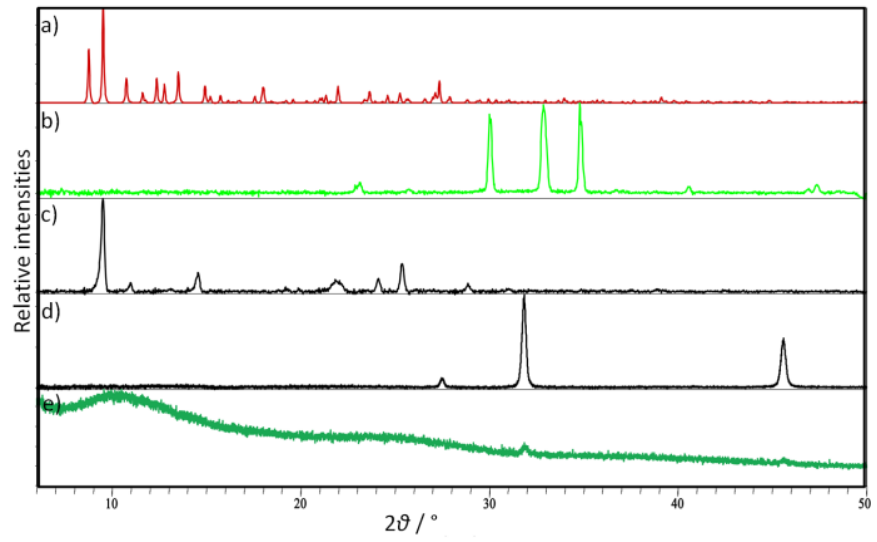

Figure S126. PXRD patterns of: a) precursor 4, b) $\mathrm{LiCl}$, c) byproduct $\mathrm{LiOAc}, \mathrm{d}$ ) resulting mixture of product $\mathbf{6}$, $\mathrm{LiCl}$, byproduct $\mathrm{LiOAc}$ and e) pure product $\mathbf{6}$ after washing with water. 


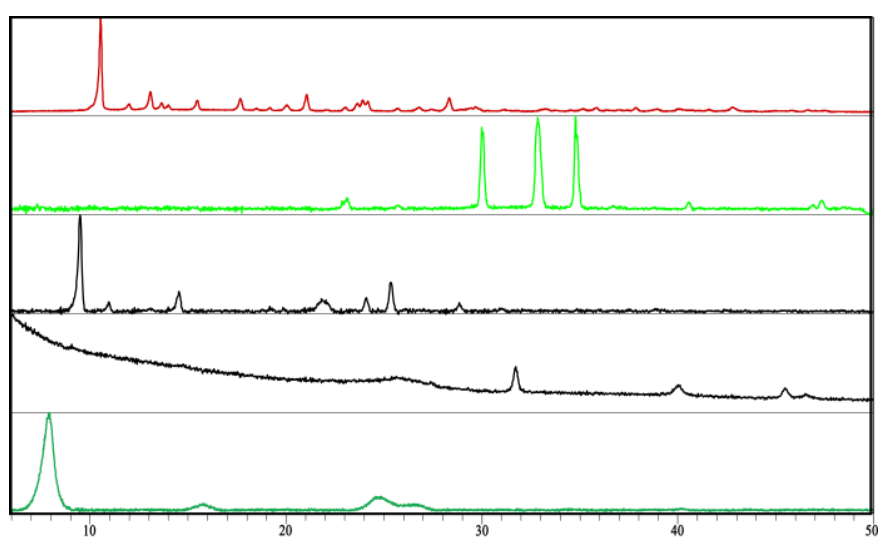

Figure 127. PXRD patterns of: a) precursor 11, b) LiCl, c) byproduct $\mathrm{LiOAc}$, d) resulting mixture of product $\mathbf{9}, \mathrm{LiCl}$, byproduct LiOAc and e) pure product 9 after washing with water.

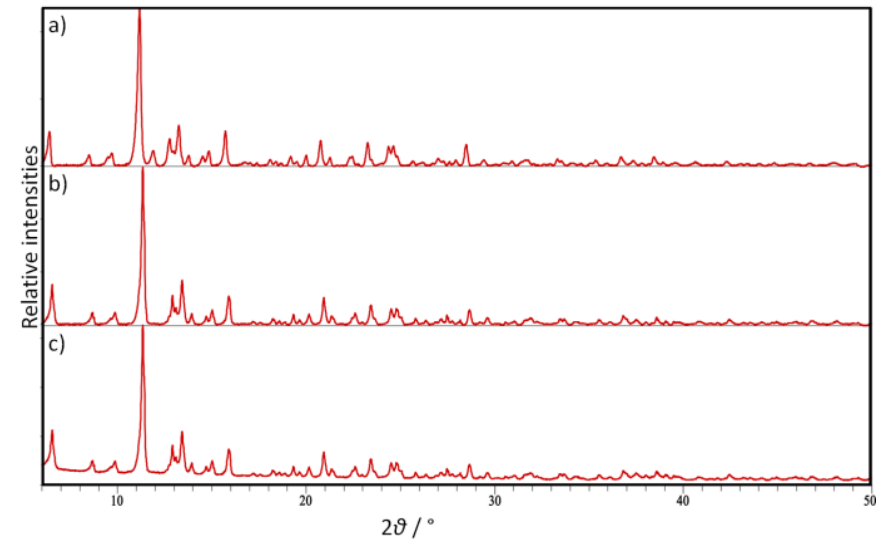

Figure S129. PXRD patterns of purified product 3 Figure S130. PXRD patterns of purified product 4 obtained by ion exchange reaction with $\mathrm{NaOAc}$ from: a) $\mathbf{1}$, b) 5 and c) 7 .

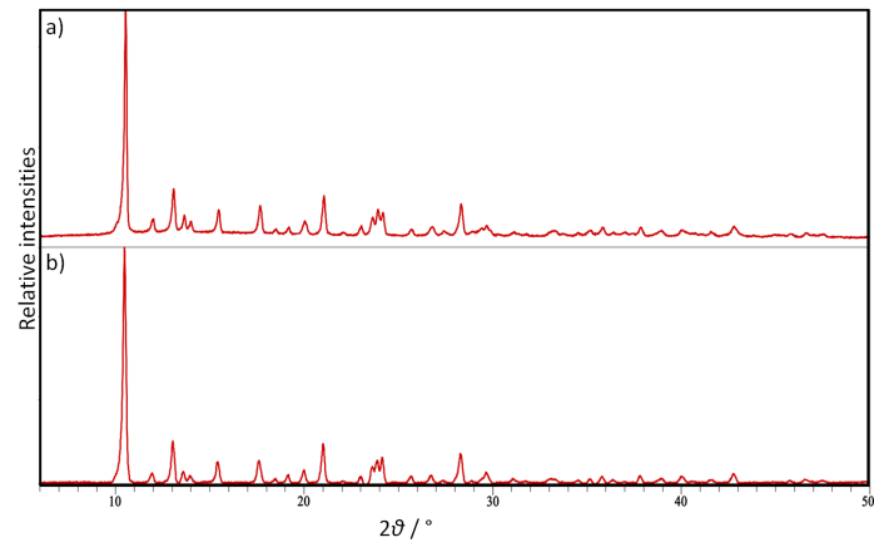

Figure S131. PXRD patterns of purified product 11 obtained by ion exchange reaction with $\mathrm{NaOAc}$ from: a) 9 and b) 13 .

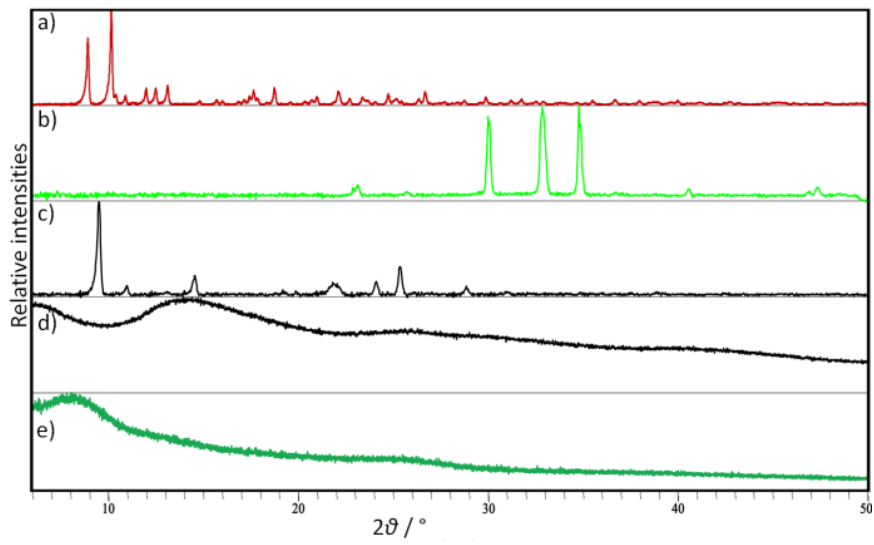

Figure 128. PXRD patterns of: a) precursor 12, b) $\mathrm{LiCl}$, c) byproduct $\mathrm{LiOAc}, \mathrm{d}$ ) resulting mixture product $\mathbf{1 0}, \mathrm{LiCl}$, byproduct LiOAc and e) pure product $\mathbf{1 0}$ after washing with water.

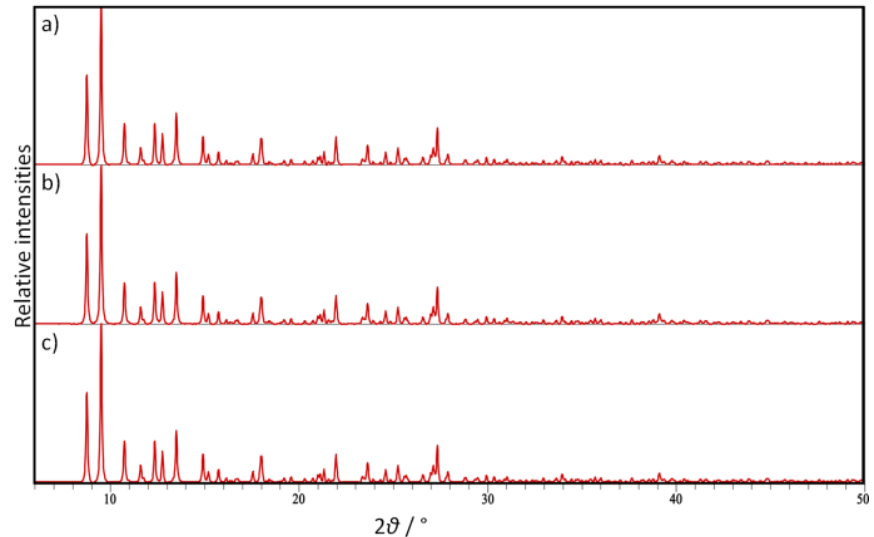

obtained by ion exchange reaction with $\mathrm{NaOAc}$ from: a) $\mathbf{2}$, b) 6 and c) 8 .

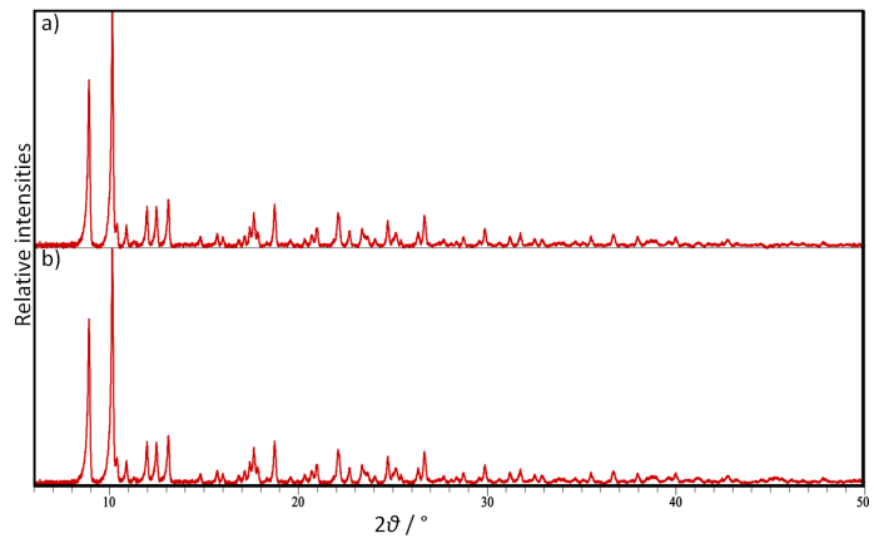

Figure S132. PXRD patterns of purified product 12 obtained by ion exchange reaction with $\mathrm{NaOAc}$ from: a) $\mathbf{1 0}$ and b) 14 . 


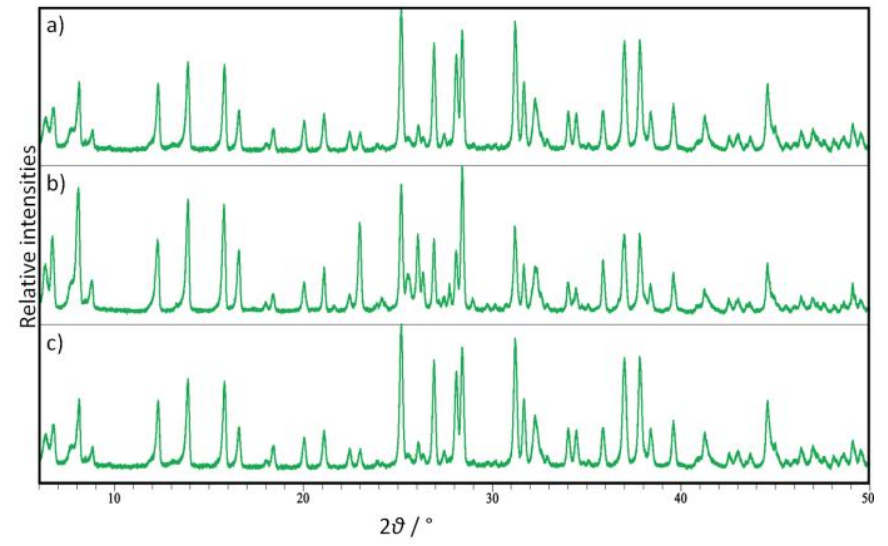

Figure S133. PXRD patterns of purified product 5 obtained by ion exchange reaction with $\mathrm{LiCl}$ from: a) 1, b) 3 and c) 7 .

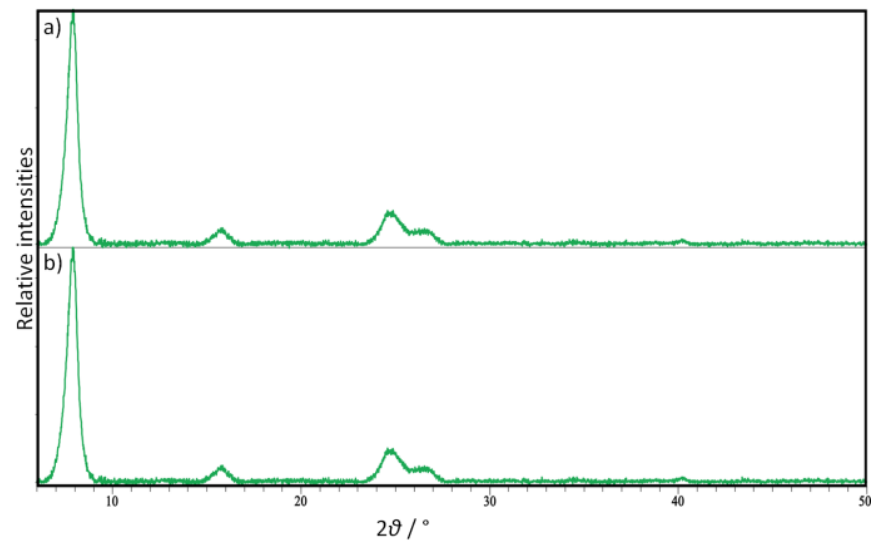

Figure S135. PXRD patterns of purified product 9 obtained by ion exchange reaction with $\mathrm{LiCl}$ from: a) $\mathbf{1 1}$ and b) $\mathbf{1 3}$.

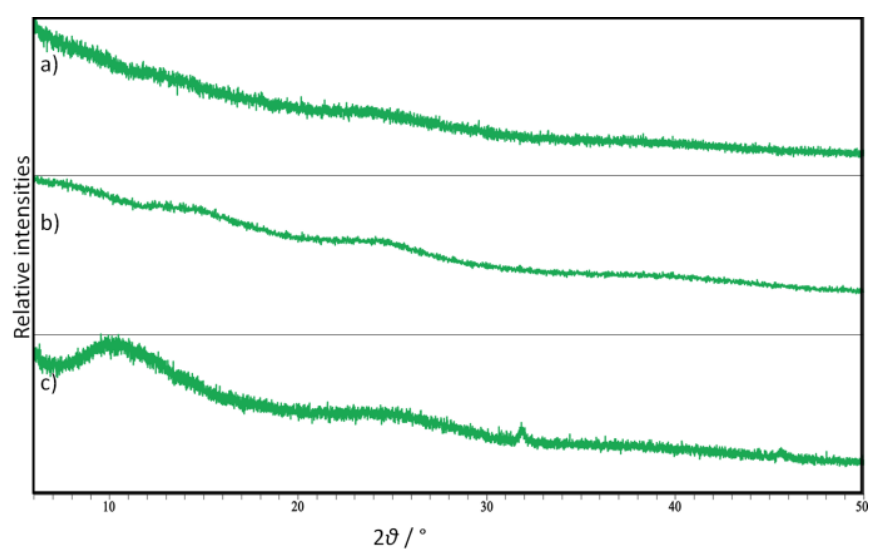

Figure S134. PXRD patterns of purified product 6 obtained by ion exchange reaction with $\mathrm{LiCl}$ from: a) 2, b) 4 and c) 8 .

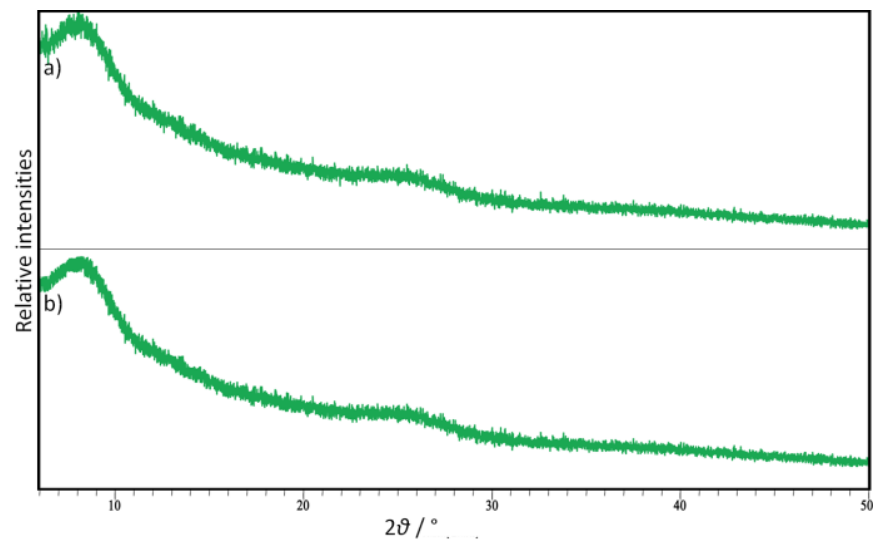

Figure S136. PXRD patterns of purified product $\mathbf{1 0}$ obtained by ion exchange reaction with $\mathrm{LiCl}$ from: a) $\mathbf{1 2}$ and b) 14 . 


\section{RAMAN SPECTROSCOPY}

Table S5. Fluorescence maxima in Raman spectra of products

\begin{tabular}{|c|c|c|c|c|c|c|c|c|}
\hline & $\mathbf{1}$ & $\mathbf{3}$ & $\mathbf{5}$ & $\mathbf{7}$ & $\mathbf{2}$ & $\mathbf{4}$ & $\mathbf{6}$ & $\mathbf{8}$ \\
\hline $\begin{array}{c}\text { Raman } \\
\text { shift } \\
/ \mathbf{c m}^{-1}\end{array}$ & $\sim 1400$ & $\sim 1250$ & $\sim 1000$ & $\sim 750$ & $\sim 400$ & $\sim 1500$ & $\sim 750$ & $\sim 250$ \\
\hline & $\mathbf{1 1}$ & $\mathbf{9}$ & $\mathbf{1 3}$ & & $\mathbf{1 2}$ & $\mathbf{1 0}$ & $\mathbf{1 4}$ \\
\hline $\begin{array}{c}\text { Raman } \\
\text { shift } \\
/ \mathbf{c m}^{-1}\end{array}$ & $\sim 2700$ & $\sim 2100$ & $\sim 2100$ & & $\sim 2250$ & $\sim 1250$ & $\sim 750$ \\
\hline
\end{tabular}

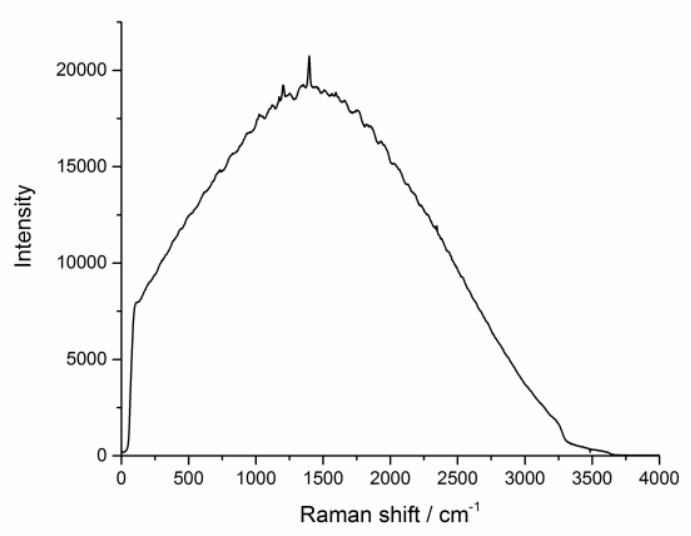

Figure S137. Raman spectrum of 1.

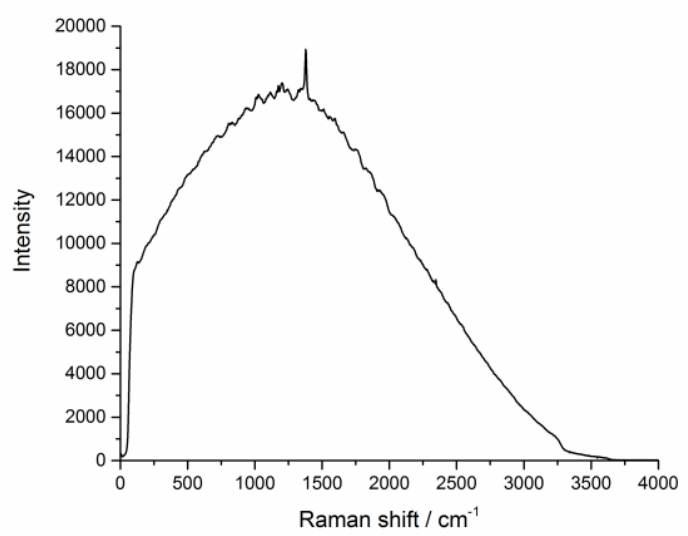

Figure S139. Raman spectrum of $\mathbf{3}$.

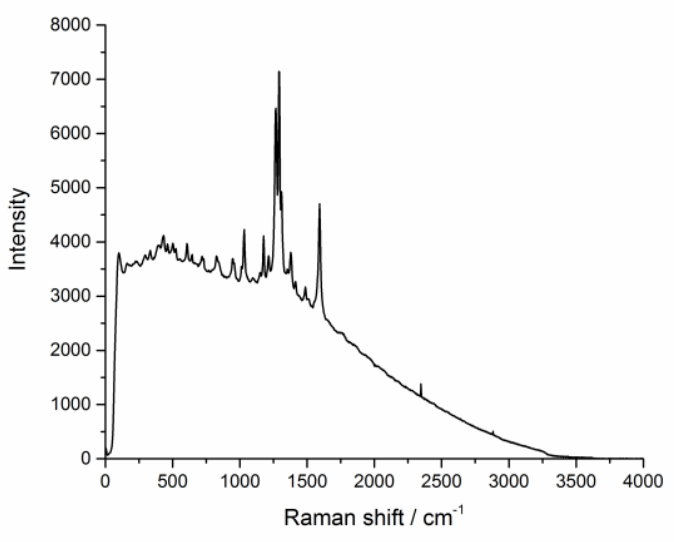

Figure S138. Raman spectrum of 2.

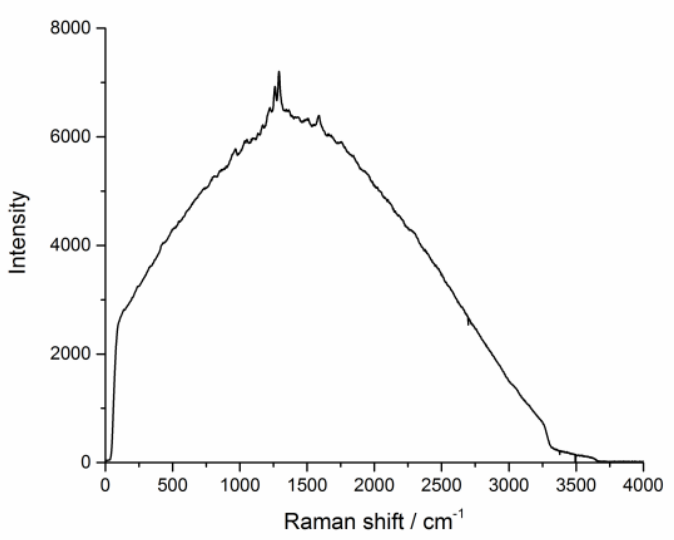

Figure S140. Raman spectrum of 4. 


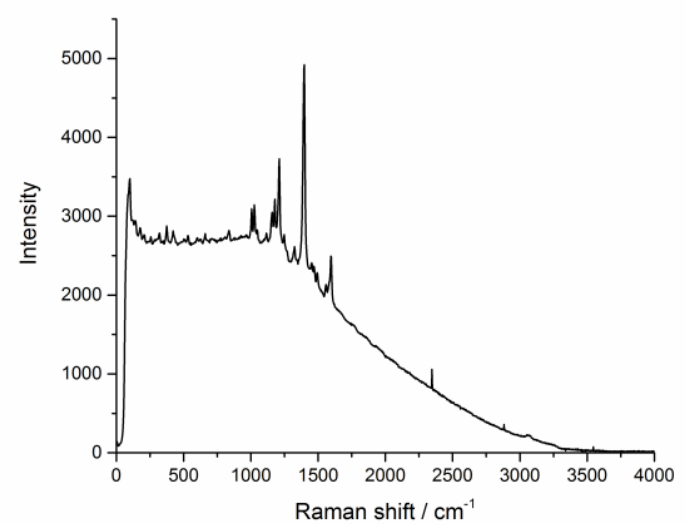

Figure S141. Raman spectrum of 5.

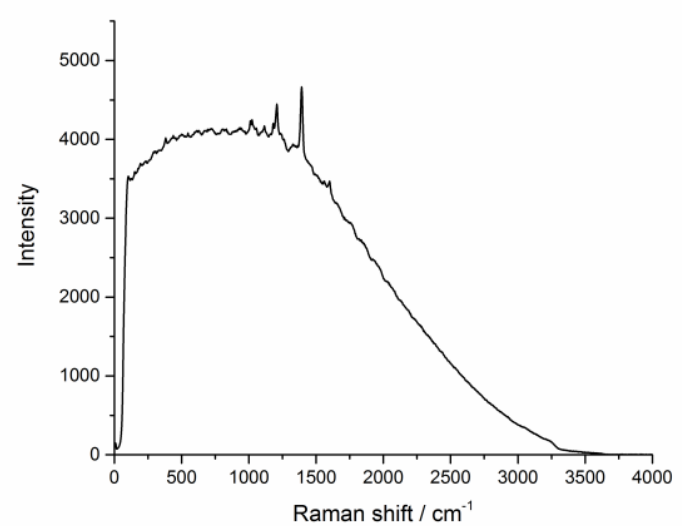

Figure S143. Raman spectrum of 7.

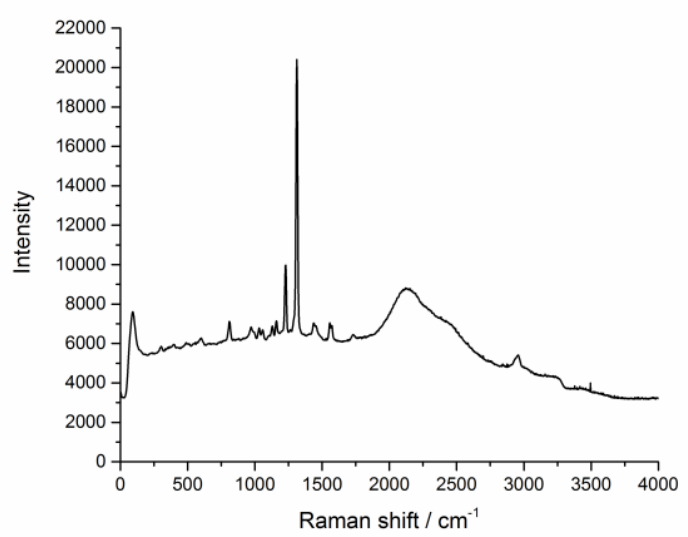

Figure S145. Raman spectrum of 9.

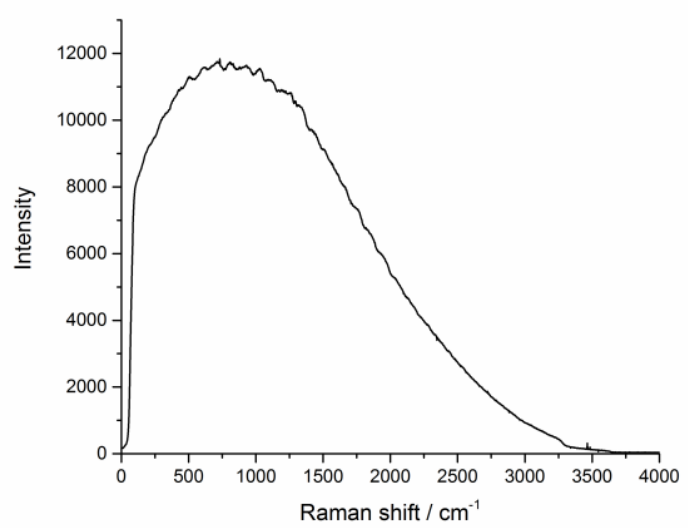

Figure S142. Raman spectrum of 6.

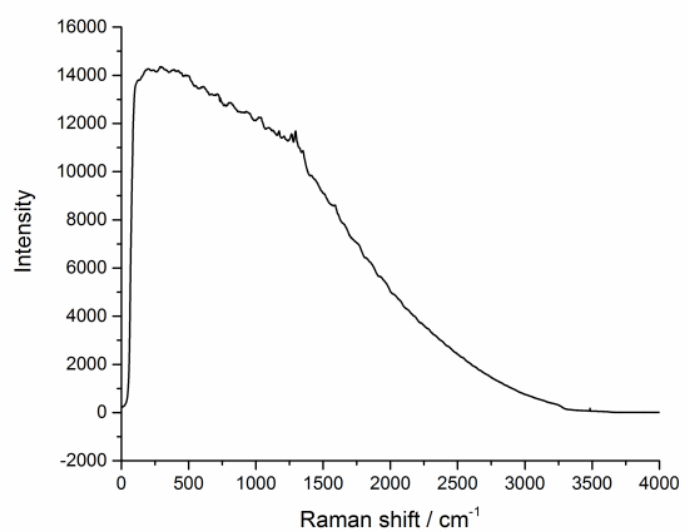

Figure S144. Raman spectrum of 8.

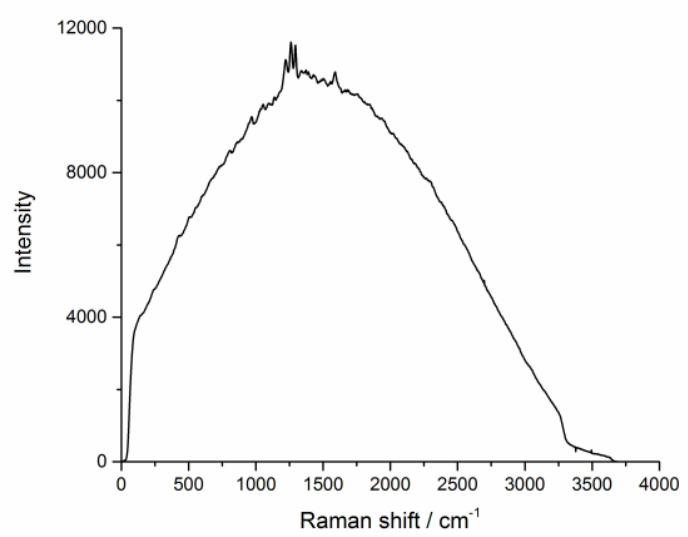

Figure S146. Raman spectrum of 10. 


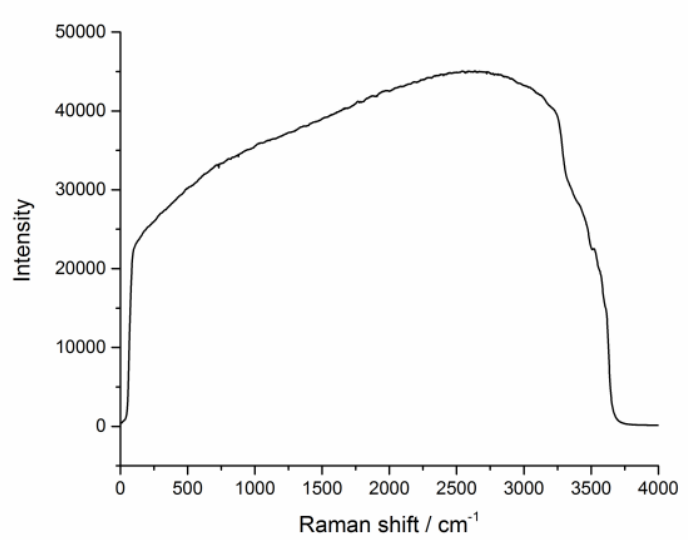

Figure S147. Raman spectrum of 11.

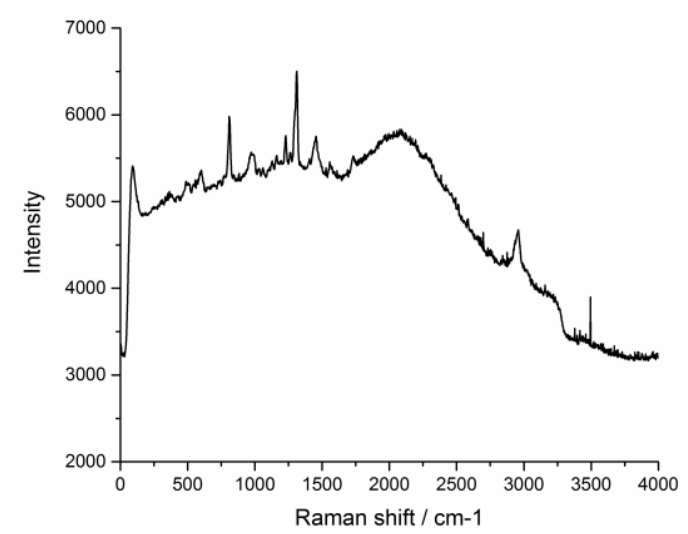

Figure S149. Raman spectrum of $\mathbf{1 3 .}$

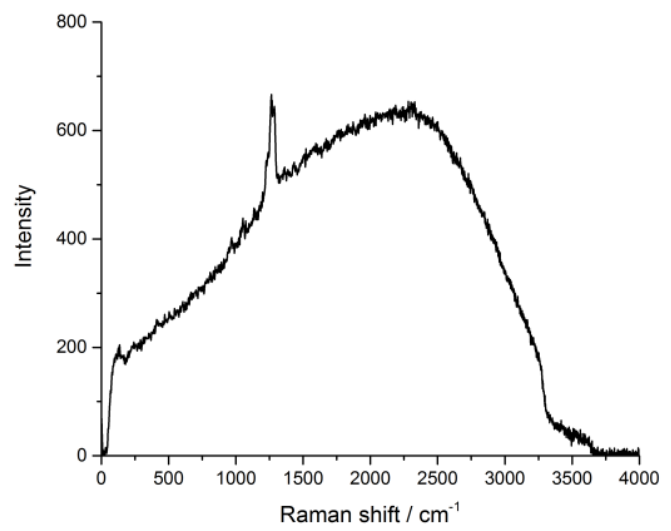

Figure S148. Raman spectrum of 12.

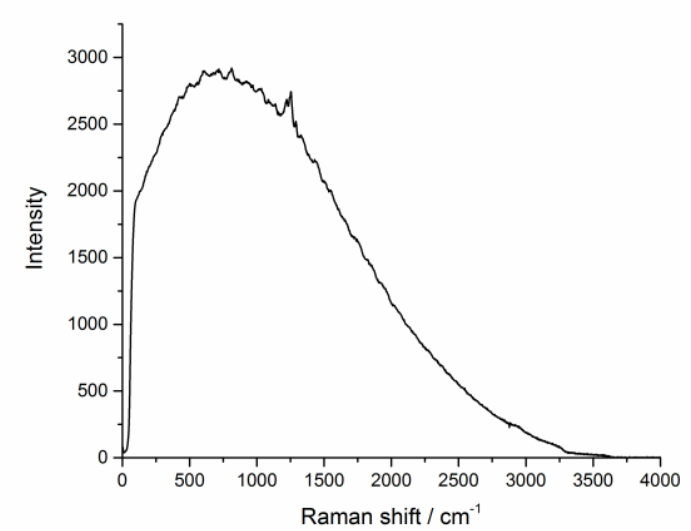

Figure S150. Raman spectrum of 14.

\section{References:}

1. (a) Juribašić, M.; Užarević, K.; Gracin, D.; Curić, M. Mechanochemical C-H Bond Activation: Rapid and Regioselective Double Cyclopalladation Monitored by in Situ Raman Spectroscopy. Chem. Commun. 2014, 50, 10287-10290; (b) Bjelopetrović, A.; Lukin, S.; Halasz, I.; Užarević, K.; Đilović, I.; Barišić, D.; Budimir, A.; Juribašić Kulcsár, Ćurić, M. Mechanism of Mechanochemical C-H Bond Activation in an Azobenzene Substrate by Pd(II) Catalysts. Chem. Eur. J. 2018, 24, 10672.

2. Monas, A.; Užarević, K.; Halasz, I.; Juribašić Kulcsár, M.; Ćurić, M. Vapour-Induced Solid-State C-H bond Activation for the Clean Synthesis of an Organopalladium Biothiol Sensor. Chem. Commun. 2016, 52, 12960-12963. 